\title{
21. NEOGENE BENTHIC FORAMINIFERS: DISTRIBUTION IN DEPTH TRAVERSE, SOUTHWEST PACIFIC 1
}

\author{
Kenji Kurihara and James P. Kennett, Graduate School of Oceanography, University of Rhode Island ${ }^{2}$
}

\begin{abstract}
Early Miocene to Quaternary benthic foraminifers have been quantitatively studied ( $>63 \mu \mathrm{m}$ size fraction) in a southwest Pacific traverse of DSDP sites at depths from about 1300 to $3200 \mathrm{~m}$ down the Lord Howe Rise (Site 590, $1299 \mathrm{~m}$; Site 591, $2131 \mathrm{~m}$; Site 206, $3196 \mathrm{~m}$ ). Benthic foraminiferal species smaller than $150 \mu \mathrm{m}$ are by far dominant in the samples, averaging from 78 to $89 \%$ of the total benthic foraminiferal assemblages in the three sites examined. Although about 150 benthic foraminiferal species or taxonomic groups have been identified, only a few species dominate the assemblages. These dominant species include Epistominella exigua, E. rotunda, and Globocassidulina subglobosa, which prevail in the three sites, and Oridorsalis umbonatus, E. umbonifera, and Cassidulina carinata, which occur usually in frequencies of between 10 and $30 \%$.

Faunal changes in Neogene benthic foraminiferal assemblages are not similar in each of the three sites, but faunal successions are most similar between the two shallowest sites. The deepest site differs in composition and distribution of dominant species. There are three intervals during which the most important changes occur in benthic foraminiferal assemblages: the early middle Miocene (14 Ma; the Orbulina suturalis Zone and the Globorotalia fohsi s.l. Zone); the late Miocene (6 Ma; the Globigerina nepenthes Zone) and near the Pliocene/Pleistocene boundary at about $2 \mathrm{Ma}$.

A Q-mode factor analysis of the faunal data has assisted in recognizing assemblage changes during the Neogene at each of the sites. Early Miocene assemblages were dominated by Globocassidulina subglobosa at Site 590 (1299 m), by G. subglobosa and Oridorsalis umbonatus at Site 591 (2131 m), and by G. subglobosa, E. exigua, and Bolivina pusilla at Site $206(3196 \mathrm{~m})$. In the early middle Miocene at Sites 590 and 591, a marked increase occurred in the frequencies of E. exigua. Epistominella exigua reached maximum abundance in the early Miocene in the deeper Site 206, and in the middle and early late Miocene in the shallower Sites 590 and 591. In the late Miocene, a spike occurred in the frequencies of $E$. umbonifera in Site 206, whereas the dominant species changed from E. exigua to $E$. rotunda at Site 590 . Latest Miocene to late Pliocene assemblages were dominated by $E$. rotunda at Site 590 , by $E$. exigua at Site 591 , and by $G$. subglobosa-E. exigua (early Pliocene) and $E$. rotunda-E. exigua (late Pliocene) at Site 206. At the Pliocene/Pleistocene boundary, $E$. exigua temporarily diminished in importance at Sites 591 and 206 . Quaternary assemblages were dominated by E. rotunda and Cassidulina carinata at Site 590, by E. rotunda at Site 591, and by E. exigua at Site 206.

These major faunal changes are all associated with known major paleoceanographic events-the middle Miocene development of the Antarctic ice sheet; the latest Miocene global cooling and increased polar glaciation; and the onset of quasiperiodic glaciation of the Northern Hemisphere. These major paleoceanographic events undoubtedly had a profound effect on the intermediate and deep water mass structure of the Tasman Sea as recorded by changes in benthic foraminiferal assemblages.
\end{abstract}

\section{INTRODUCTION}

Early Miocene to Quaternary benthic foraminifers have been studied from three sites in the Tasman Sea, southwest Pacific: Site $590\left(31^{\circ} 10.02^{\prime} \mathrm{S}, 163^{\circ} 21.51^{\prime} \mathrm{E}\right)$, Site $591\left(31^{\circ} 35.06^{\prime} \mathrm{S}, 164^{\circ} 26.92^{\prime} \mathrm{E}\right)$, both DSDP Leg 90 , and Site $206\left(32^{\circ} 00.75^{\prime} \mathrm{S}, 165^{\circ} 27.15^{\prime} \mathrm{E}\right)$, DSDP Leg 21 . Sites 590 and 591 were cored on the Lord Howe Rise in water depths of $1299 \mathrm{~m}$ and $2131 \mathrm{~m}$ respectively. Site 206 is located in the New Caledonia Basin to the east of the Lord Howe Rise at greater water depth $(3196 \mathrm{~m})$.

We selected these three sites because they form a suite of cores ranging from intermediate to deep water between about $1000 \mathrm{~m}$ and $3000 \mathrm{~m}$. The purpose of this study was to examine changes in benthic foraminiferal assemblages at different water depths during the Neogene. This, in turn, should provide information about paleoceanographic changes in the vertical water mass

\footnotetext{
${ }^{1}$ Kennett, J. P., von der Borch, C. C., et al., Init, Repts. DSDP, 90: Washington (U.S. Govt, Printing Office).

2 Addresses: (Kurihara, permanent address) Rikkyo (St. Paul's) University, Nishi-ikebukuro, Tokyo, Japan; (Kennett) Graduate School of Oceanography, University of Rhode 1sland, Kingston, RI 02881.
}

structure of the Tasman Sea. Changes in benthic foraminifers are known to be associated with certain deep and intermediate water masses (Douglas and Woodruff, 1981). Changes in assemblages through geologic time have also been shown to be sensitive monitors of changes in the distribution of deep and bottom water masses (Schnitker, 1974; Streeter, 1973; and others).

The three sequences are also appropriate for this investigation for several other reasons: (1) they all consist of continuous or nearly continuous early Miocene to Quaternary sequences; (2) the three sections are all well correlated using the same planktonic foraminiferal zonation (Kennett and Srinivasan, 1983); (3) the three sites are located above the foraminiferal lysocline and yield rich assemblages of benthic foraminifers throughout; and (4) no displaced benthic faunas were recognized in any of the sites.

\section{METHOD OF STUDY}

Benthic foraminifers were studied from $\sim 9 \mathrm{~m}$ intervals from 149 core-catcher samples: 51 from Site 590, 55 from Site 591, and 43 from Site 206 (Fig. 2). The core-catcher samples were washed over a $63 \mu \mathrm{m}$ Tyler screen. Benthic foraminiferal assemblages were quantitatively examined from the $>63 \mu \mathrm{m}$ fraction, chosen because relatively small individuals between 63 and $150 \mu \mathrm{m}$ completely dominate the assemblages. 


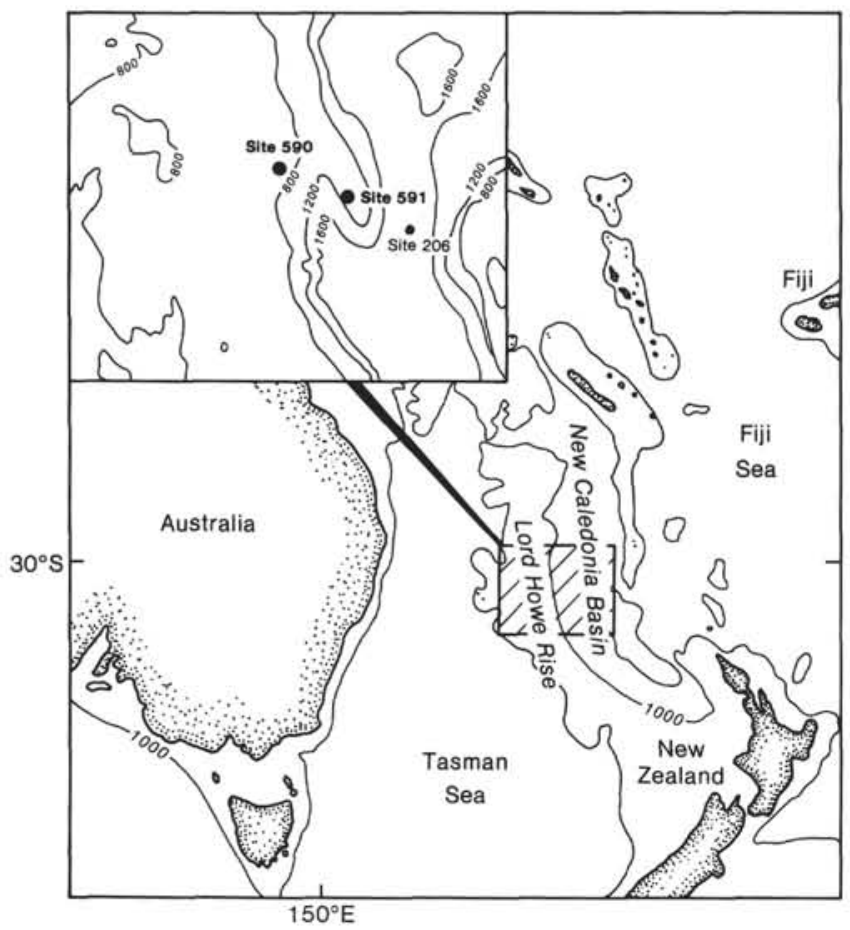

Figure 1. Location of DSDP Sites 590, 591, and 206 on the Lord Howe Rise and in the New Caledonia Basin. Depth is shown in fathoms.

Different workers have studied rather different size fractions of benthic foraminiferal assemblages. For example, fractions greater than $300 \mu \mathrm{m}$ were studied by Lutze (1979); $>250 \mu \mathrm{m}$ by Lohmann (1978); $>150 \mu \mathrm{m}$ by Woodruff and Douglas (1981); $>125 \mu \mathrm{m}$ by Schnitker (1979); $>100 \mu \mathrm{m}$ by Douglas (1973); $>73 \mu \mathrm{m}$ by Boltovskoy (1983); and $>62 \mu \mathrm{m}$ by Resig (1976). Unfortunately, the size distribution of benthic foraminiferal tests has not previously been examined in deepsea sediments. We examined the relative abundances of benthic foraminiferal tests larger and smaller than $150 \mu \mathrm{m}$. A number of important studies of Recent deep-sea benthic foraminiferal distribution patterns have been analyzed from the $>150 \mu \mathrm{m}$ size fractions (Parker, 1958; Phleger et al., 1953). Foraminiferal tests smaller than $150 \mu \mathrm{m}$ range from 50 to $99 \%$ of the assemblages (Fig. 3), and average 78 to $89 \%$ of the assemblages in the three sites examined:

\begin{tabular}{lcc}
\hline Site & $>150 \mu$ m (avg.) $(\%)$ & $<150 \mu$ m (avg.) (\%) \\
\hline 590 & $1-35(11)$ & $65-99(89)$ \\
591 & $8-50(22)$ & $50-92(78)$ \\
206 & $5-37(15)$ & $63-95(85)$ \\
\hline
\end{tabular}

The number of individuals $>150 \mu \mathrm{m}$ were not always sufficiently abundant to allow a quantitative study of this size fraction alone.

Washed samples were split to a size containing about 150 to 300 individuals using a microsplitter. The split samples were spread on a counting tray, and all benthic foraminifers were removed and mounted in taxonomic groups on slides. The procedure of spreading samples and removing benthic foraminifers was repeated three times or more for each sample to insure that no specimens were missed. The average number of benthic foraminifers counted was 164 individuals per sample at Site 590, 212 at Site 591, and 224 at Site 206.

Planktonic to benthic foraminiferal ratios (P/B ratios) were also determined in the fractions larger than $63 \mu \mathrm{m}$. In order to estimate the number of planktonic foraminifers contained in the samples, the samples used for benthic forms were microsplit, and 300 to 1000 planktonic individuals were counted.

The data for each site were also separately analyzed using Q-mode factor analysis (Klovan and Imbrie, 1971) to assist in evaluating the major changes in faunal components within each site.

\section{RESULTS}

\section{Site $\mathbf{5 9 0}$}

Site 590 lies near the crest of the Lord Howe Rise at a water depth of $1299 \mathrm{~m}$ and is the shallowest of the three sites studied. The section is a 499 m-thick sequence of mostly foraminifer-bearing nannofossil ooze from early Miocene (Catapsydrax dissimilis Zone) to Quaternary (Globorotalia truncatulinoides Zone). An unconformity exists at the early/middle Miocene boundary where the Praeorbulina glomerosa curva and Orbulina suturalis Zones are missing.

Benthic foraminifers were studied from 51 samples of Hole 590 (590-1, CC to 3,CC), Hole 590A (590A-1, CC to $27, \mathrm{CC}$ ), and Hole 590B (590B-30, CC to 53, CC) (Fig. 2). Benthic foraminifers are well preserved in the Quaternary to late Miocene, but are recrystallized during most of the middle and early Miocene. There is a large range of planktonic to benthic foraminiferal ratios, from $45: 1$ to $4469: 1$ (Fig. 4). The largest ratios occur in the early Miocene and decrease over the early/middle Miocene boundary. For the remainder of the Miocene to the Quaternary, the ratios are rather constant and relatively low, mostly within the range of $50: 1$ to $300: 1$.

The stratigraphic distribution of benthic foraminifers in Site 590 is shown in Table 1. Benthic foraminiferal faunas are dominated by Epistominella, Globocassidulina, Cassidulina, Bolivina, Oridorsalis, and Gyroidina. Most of the abundant species range throughout the section from the early Miocene to the Quaternary. There are many species with rare or sporadic occurrences (Table 1) and hence their stratigraphic ranges are difficult to determine with accuracy.

Early Miocene assemblages at Site 590 were dominated by Globocassidulina subglobosa (Brady). Other important species include Oridorsalis umbonatus (Reuss), Cibicidoides mundulus (Brady, Parker, and Jones) and Bolivina pusilla Schwager. In one sample within the Catapsydrax dissimilis Zone, Bolivina sp. A was found with exceptionally high frequencies $(48 \%)$.

In the middle Miocene, the benthic foraminiferal assemblages were dominated by Epistominella exigua (Brady) usually in frequencies between 10 and $25 \%$. Other important species include Epistominella rotunda (Husezima and Maruhasi), Cassidulina sp. A, B. pusilla, O. umbonatus, G. subglobosa, and Cibicidoides wuellerstorfi (Schwager). Globocassidulina subglobosa, which was the dominant species of the early Miocene assemblages, tended to decrease in abundance during the middle Miocene.

Late Miocene assemblages were also dominated by $E$. exigua. The frequencies of this dominant species decrease in the Globigerina nepenthes Zone and E. rotunda was dominant in the late late Miocene. Other important species include Cassidulina sp. A, B. pusilla, and O. umbonatus. At the same time as the dominant species changed from $E$. exigua to $E$. rotunda, several species showed interesting occurrences in the late Miocene. Rectuvigerina sp. appears only in the Neogloboquadrina continuosa and Globigerina nepenthes zones. Pyrgo and Quinqueloculina first occur in the G. nepenthes Zone and Globorotalia conomiozea Zone, respectively. Bulimina stri- 
Site $\mathbf{5 9 0}$

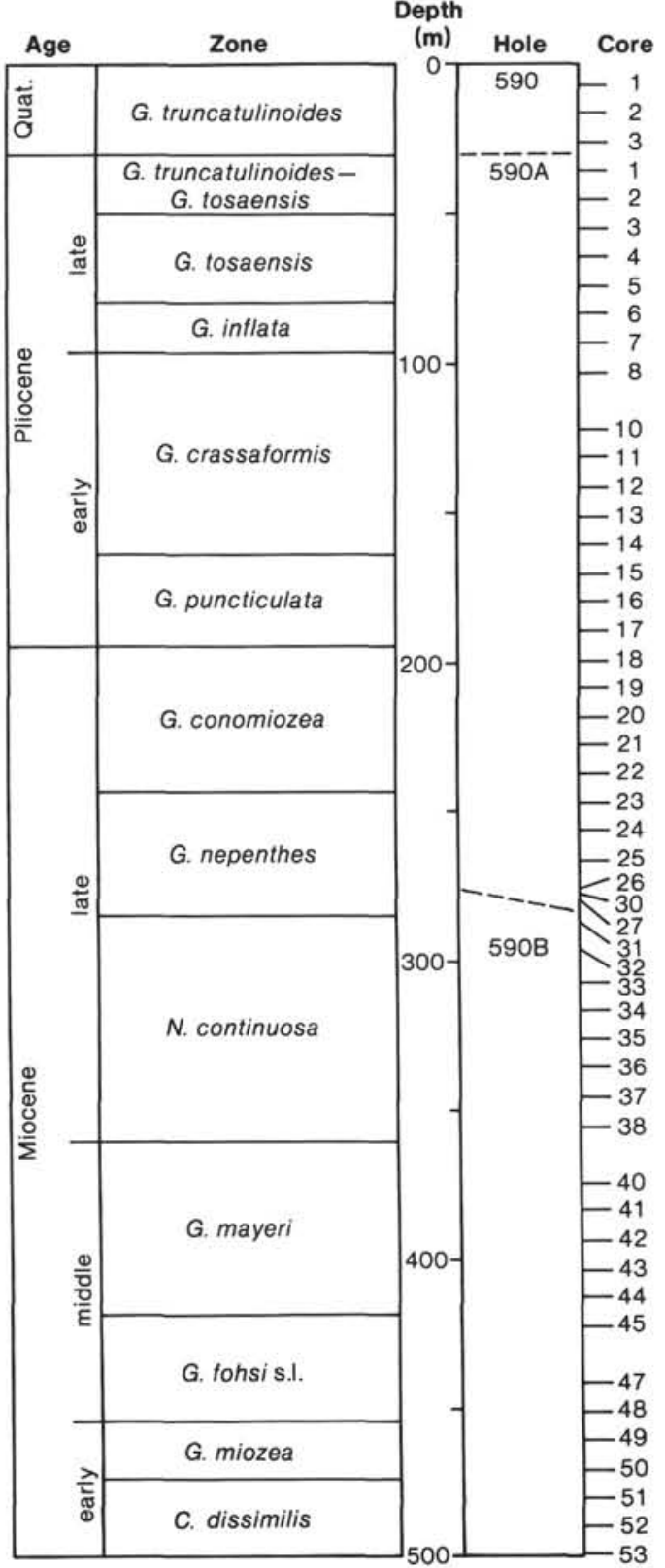

Site $\mathbf{5 9 1}$

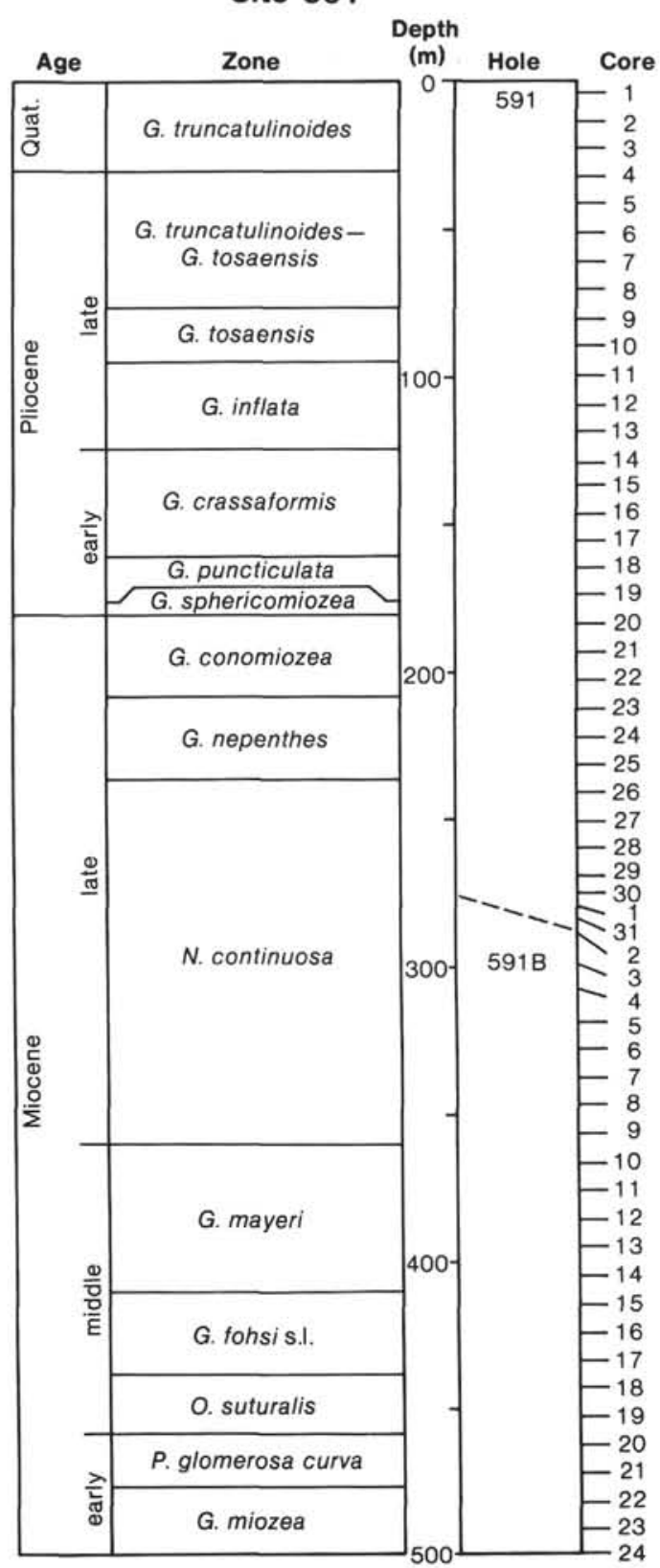

Site 206

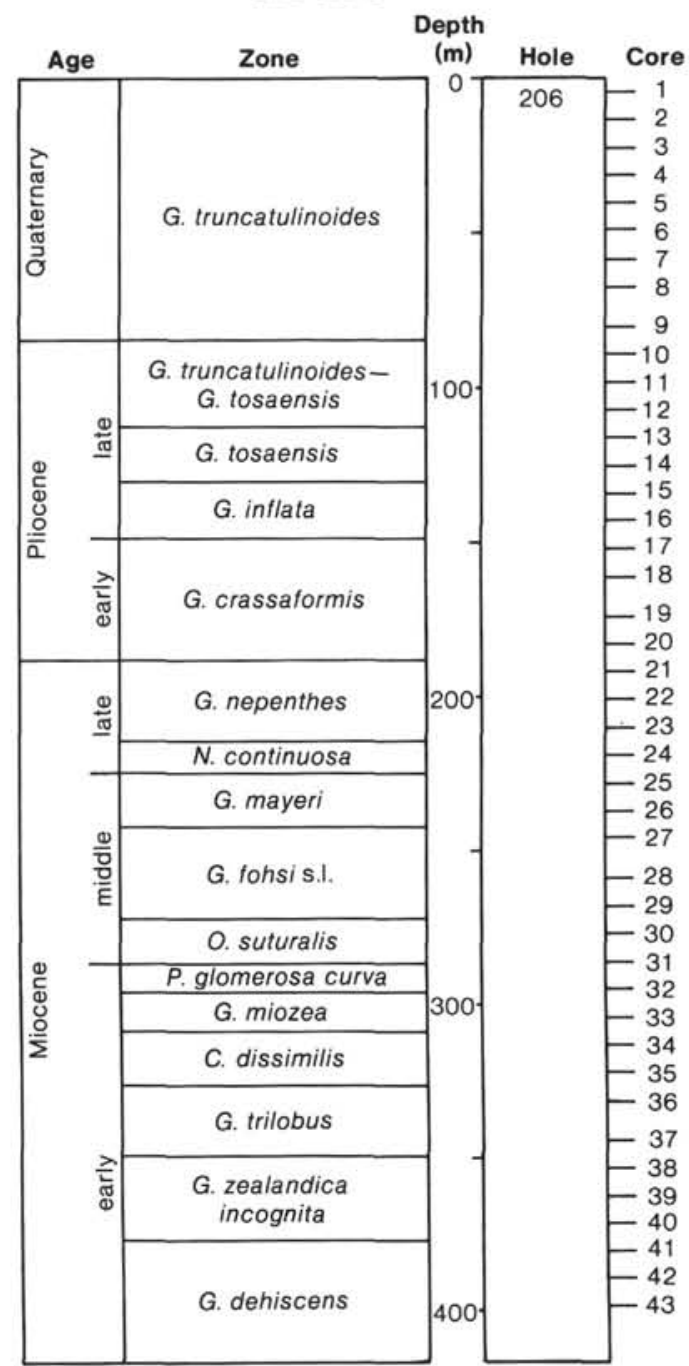




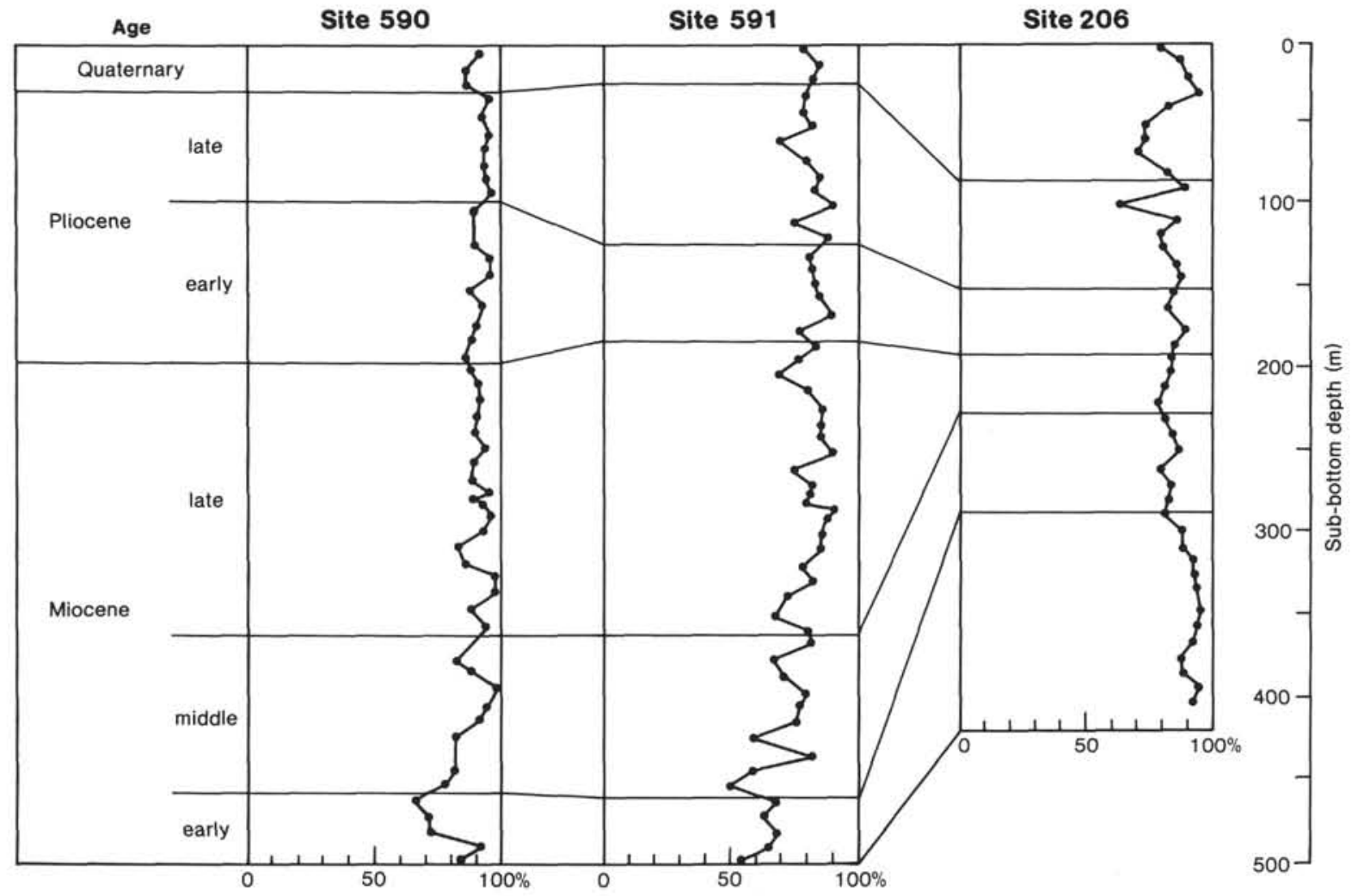

Figure 3. Percentages of benthic foraminifers $<150 \mu \mathrm{m}$ within the total benthic assemblages at Sites 590, 591, and 206.

ata d'Orbigny and Epistominella rugosa (Phleger and Parker) temporarily disappear in the upper part of the Globigerina nepenthes Zone, while Uvigerina peregrina Cushman first appears within this zone.

Pliocene and Quaternary assemblages were dominated by $E$. rotund $a$, usually with frequencies between 10 and $25 \%$, and the late Quaternary assemblage was dominated by Cassidulina carinata Silvestri. Other important species include E. exigua, Cassidulina sp. A, Globocassidulina subglobosa, Bolivina decussata Brady, Virgulopsis sp., Gyroidina cf. quinqueloba Uchio and Anomalinoides sp. B. Globocassidulina subglobosa increased again in the latest Pliocene and Quaternary. The changing species dominance of benthic foraminiferal assemblages for Site 590 is summarized in Figure 5.

\section{Site 591}

Site 591 lies near the crest of a southern spur on the eastern part of the Lord Howe Rise at a water depth of $2130 \mathrm{~m}$. The crest of the spur, separated from the main part of the Lord Howe Rise by a valley, is largely isolated from sediment transportation from the main part of the Lord Howe Rise. The sedimentary sequence recovered at Site 591 is a calcareous biogenic facies consisting mainly of calcareous nannofossil and foraminiferal ooze. It is a 500-m-thick continuous sequence from the late early Miocene (Globorotalia miozea Zone) to the Quaternary (Globorotalia truncatulinoides Zone).

Fifty-five samples were studied from Hole 591 (591$1, \mathrm{CC}$ to $591-31, \mathrm{CC})$ and Hole 591B (591B-1,CC to 591B-24,CC), as shown in Figure 2. Benthic foramini- fers are well preserved in the upper part of the sequence, from Quaternary to late Miocene in age. In the early and middle Miocene, benthic foraminifers are rarer than in sediments of younger age. Recrystallization of benthic foraminiferal tests at Site 591 becomes conspicuous, especially during the early Miocene. $\mathrm{P} / \mathrm{B}$ ratios are higher than at the other two sites (Fig. 4) ranging from 179:1 to 10674:1. Maximal P/B values occur in the early Miocene and average about 6400:1 in this interval. As in Site 590, P/B ratios decrease near the early/middle Miocene boundary. Beginning in the early middle Miocene, the ratios become less than 100:1, with several peaks of up to about 2000:1 to $3000: 1$.

Table 2 shows the stratigraphic distribution of benthic foraminifers at Site 591. The following species are the most abundant at this site: Epistominella exigua, $E$. rotunda, Globocassidulina subglobosa, Cassidulina $\mathrm{sp}$. A, Oridorsalis umbonatus, Bolivina decussata, B. pusilla, Gyroidina sp. A, Virgulopsis sp., Cibicidoides wuellerstorfi, Pullenia osloensis Feyling-Hanssen, and Siphonaperta sp.

Early Miocene assemblages were dominated by Globocassidulina subglobosa and Oridorsalis umbonatus, with frequencies up to 31 and $20 \%$, respectively.

In the middle Miocene, the assemblages were dominated by $E$. exigua. This species increased in frequencies in the early middle Miocene as in Site 590. Other important species include $G$. subglobosa, O. umbonatus, Cassidulina sp. A, Cibicidoides wuellerstorfi, C. cf. opacus (Carter), and Virgulopsis sp.

Late Miocene and Pliocene assemblages were also dominated by Epistominella exigua. This dominant species 


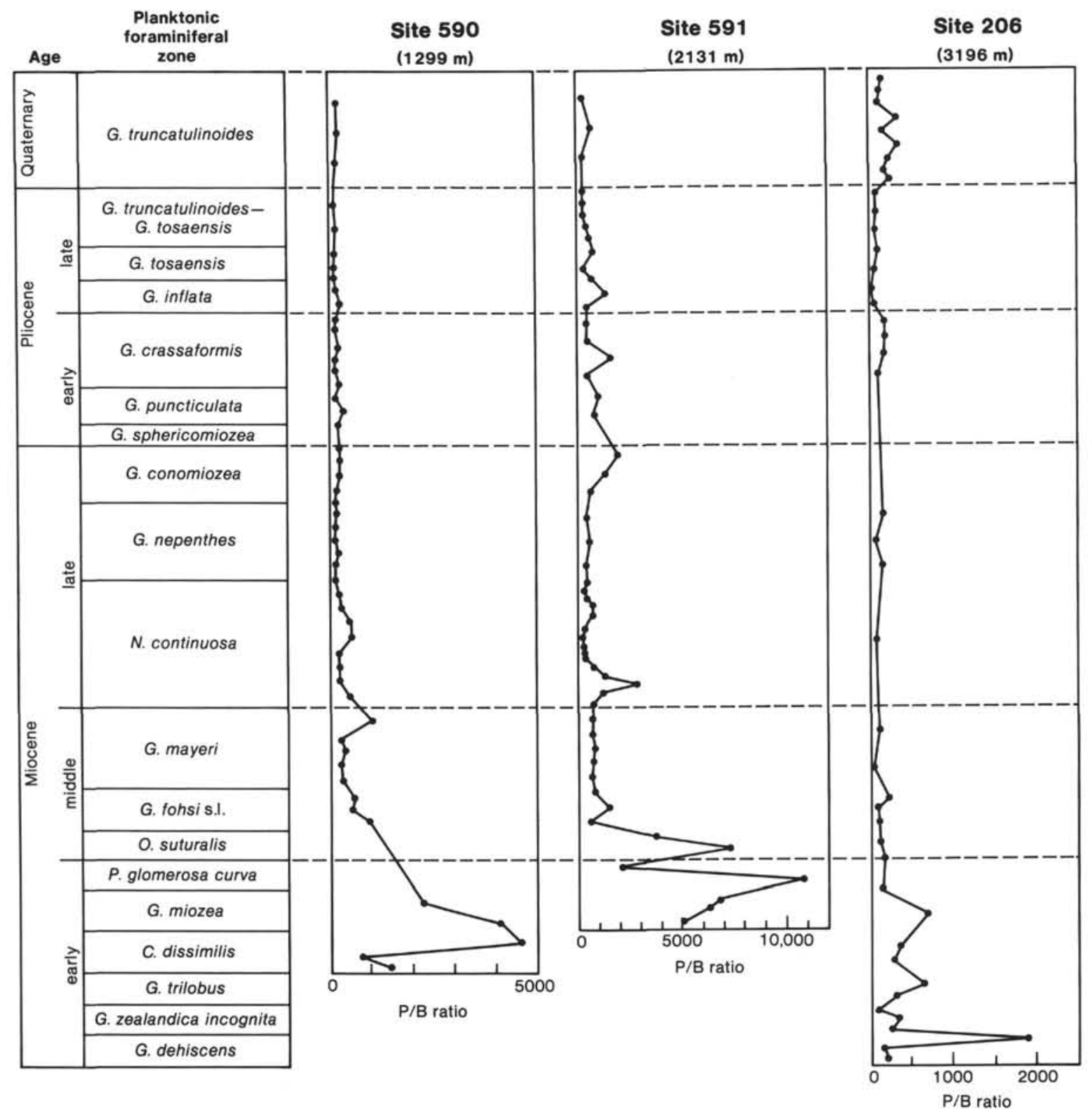

Figure 4. Planktonic to benthic foraminiferal ratios (P/B ratios) at Sites 590, 591, and 206 (water depth in parentheses).

showed highest frequencies in the early late Miocene (15 to $30 \%$ ). Other important species include $G$. subglobosa, O. umbonatus, $E$. rotunda, Cassidulina sp. A, Siphonaperta sp., and B. decussata. Quinqueloculina and Pyrgo were found to occur beginning in the Neogloboquadrina continuosa Zone, as in Site 590 , but there are some sporadic occurrences in the early and middle Miocene.

Quaternary assemblages were dominated by $E$. rotun$d a$. Near the Pliocene/Quaternary boundary, E. exigua temporarily decreased in frequency and was even absent just below the boundary $(591-4, C C)$. Epistominella rotunda and $O$. umbonatus also increased in frequency near the boundary and remained important throughout the Quaternary. Other important species are similar to those of late Miocene and Pliocene age. The changing species dominance of benthic foraminiferal assemblages for Site 591 is summarized in Figure 5.
Site 206

Site 206 is located about $220 \mathrm{~km}$ to the southeast of Site 590. It lies at the bottom of the New Caledonia Basin, and is the deepest of the three sites studied (3196 m). The Neogene of Site 206 is represented by $440 \mathrm{~m}$ of foraminifer-rich nannofossil ooze. Planktonic foraminifers at this site were studied by Kennett (1973) and Srinivasan and Kennett (1981), and 16 planktonic foraminiferal zones were well recognized, from the Globoquadrina dehiscens Zone of the early early Miocene to the Globorotalia truncatulinoides Zone of the Quaternary. There is an unconformity between the late Miocene and the early Pliocene, where the $G$. conomiozea Zone to the $G$. puncticulata Zone are missing.

Benthic foraminifers were studied from 43 samples of Hole 206 (206-1,CC to 43,CC). They are not as well preserved as they are at the other two sites, but rich calcare- 
Table 1. Distribution of benthic foraminifers at Site 590 .

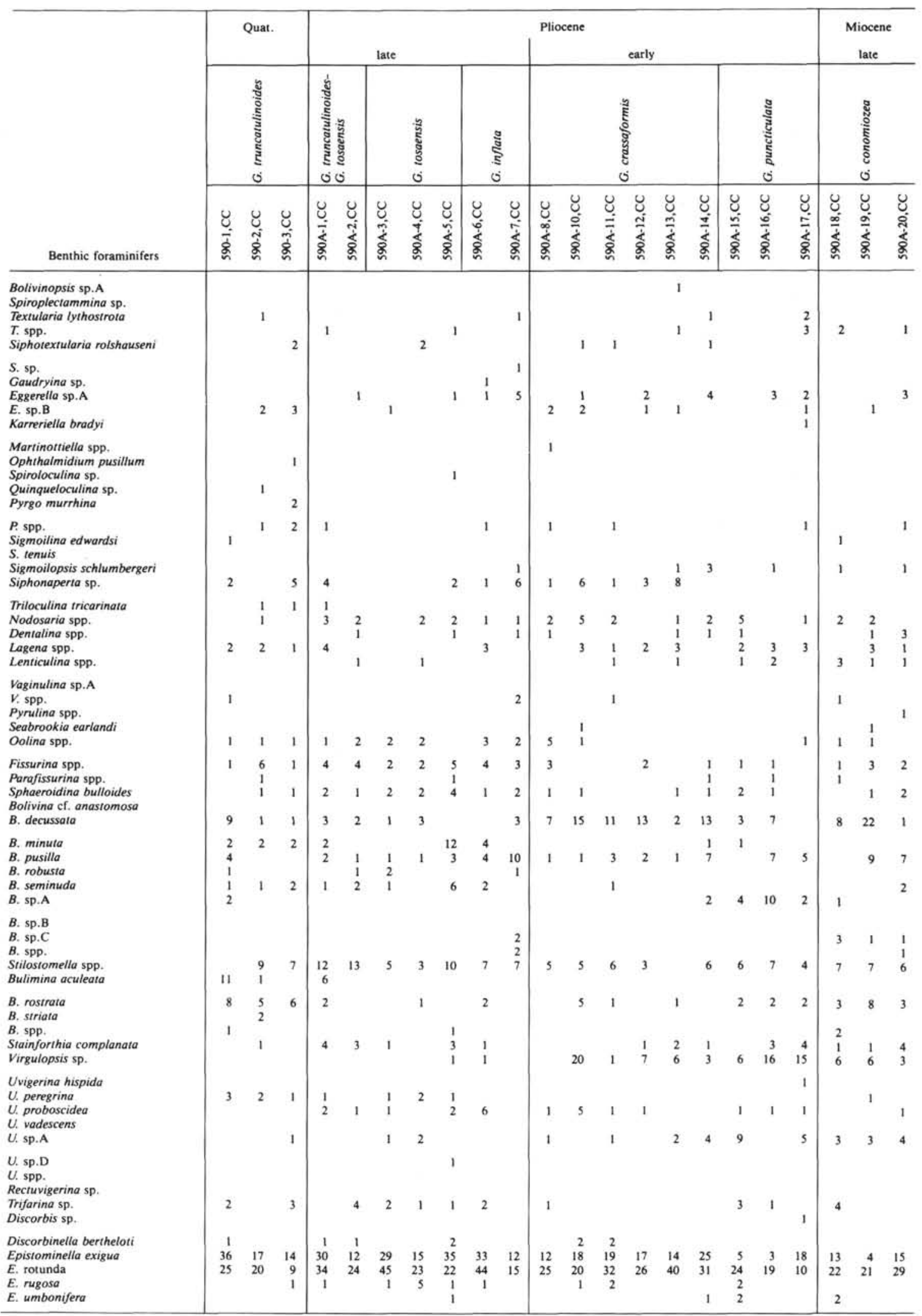

Note: Number represents no. specimens counted in $>63 \mu \mathrm{m}$. Planktonic foraminifer zones and DSDP sample nos, in column heads. 
Table 1. (Continued).

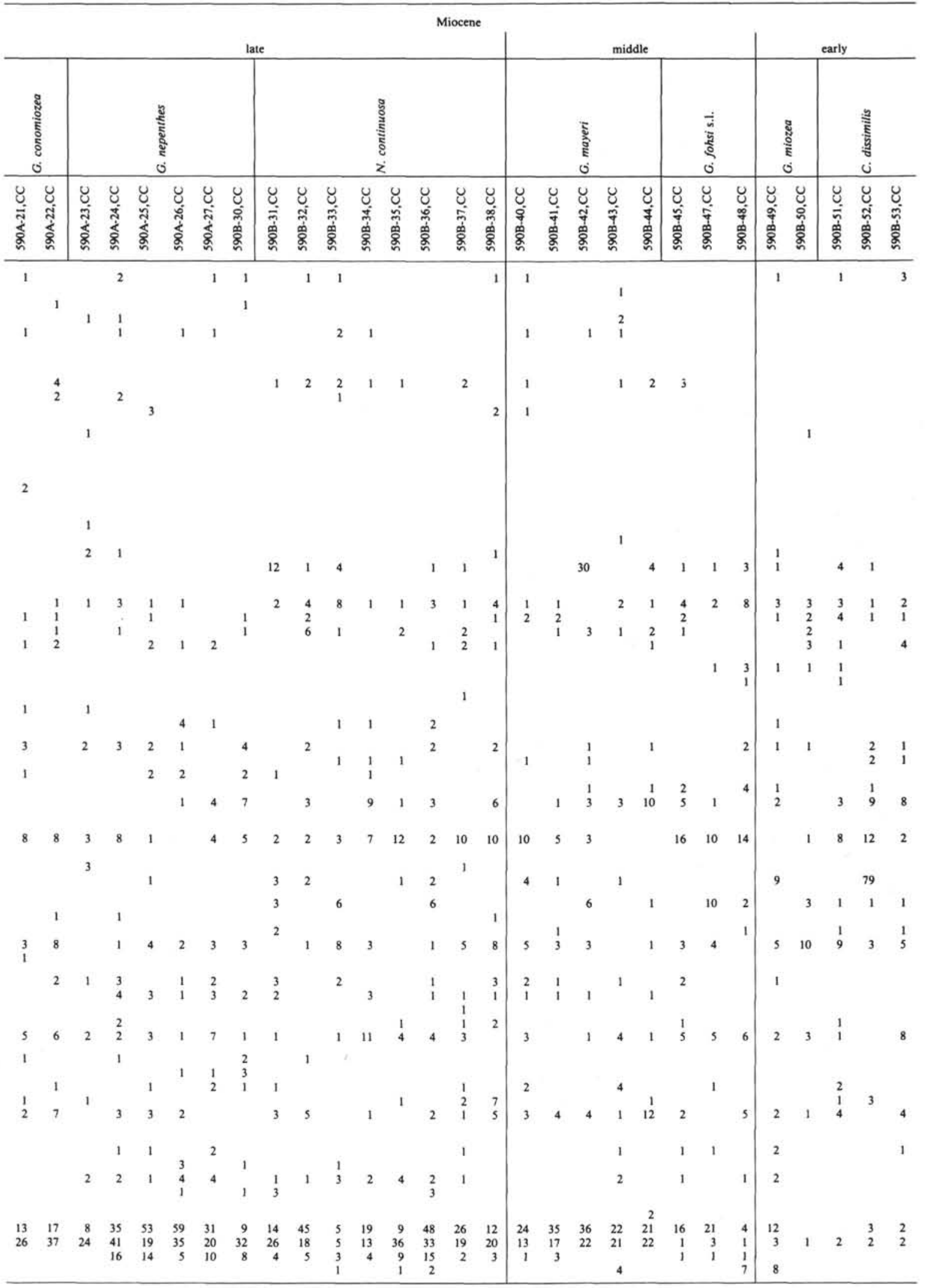


Table 1. (Continued).

\begin{tabular}{|c|c|c|c|c|c|c|c|c|c|c|c|c|c|c|c|c|c|c|c|c|c|c|}
\hline \multirow[b]{3}{*}{ Benthic foraminifers } & \multicolumn{3}{|c|}{ Quat. } & \multicolumn{16}{|c|}{ Pliocene } & \multicolumn{3}{|c|}{$\begin{array}{c}\text { Miocene } \\
\text { late }\end{array}$} \\
\hline & & 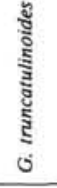 & & 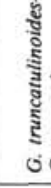 & 心 & & 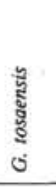 & & sัँ & & & & & हू. & & & & 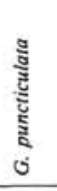 & & & 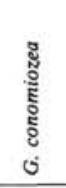 & \\
\hline & 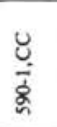 & 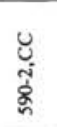 & 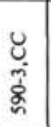 & $\frac{\text { U }}{\frac{\delta}{\delta}}$ & 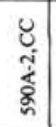 & 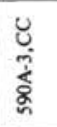 & 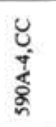 & 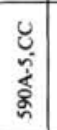 & $\begin{array}{l}\text { Uू } \\
\vdots \\
\vdots \\
\vdots\end{array}$ & 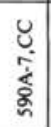 & $\begin{array}{l}0 \\
0 \\
0 \\
\vdots \\
\vdots \\
\vdots\end{array}$ & 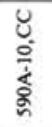 & 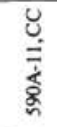 & 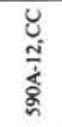 & 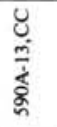 & 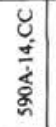 & 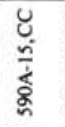 & 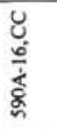 & \begin{tabular}{l|} 
\\
0 \\
$\vdots$ \\
$\vdots$ \\
$\delta$
\end{tabular} & 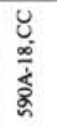 & $\frac{u}{0}$ & 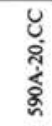 \\
\hline $\begin{array}{l}\text { E. sp. } \\
\text { Gavelinopsis lobatulus } \\
\text { Laticarinina alto-camerata } \\
\text { L. pauperata } \\
\text { Valvulineria sp. }\end{array}$ & 6 & 7 & 8 & $\begin{array}{l}4 \\
1\end{array}$ & 3 & 1 & 2 & 2 & 9 & 10 & 8 & 8 & & & & 1 & 3 & 2 & 2 & 1 & 1 & 15 \\
\hline $\begin{array}{l}\text { Heronallenia sp.A } \\
\text { Siphonina australis } \\
\text { Pleurostomella spp. } \\
\text { Cassidella bradyi } \\
\text { Cassidulina carinata }\end{array}$ & 43 & $\begin{array}{l}2 \\
2\end{array}$ & $\begin{array}{l}2 \\
4 \\
1 \\
1\end{array}$ & $\begin{array}{r}1 \\
2 \\
11\end{array}$ & $\begin{array}{l}1 \\
1\end{array}$ & 1 & 2 & $\begin{array}{l}1 \\
3\end{array}$ & 6 & 4 & 2 & 6 & $\begin{array}{l}1 \\
1\end{array}$ & $\begin{array}{l}1 \\
1\end{array}$ & $\begin{array}{l}2 \\
1\end{array}$ & 2 & 1 & 5 & & 4 & 1 & 8 \\
\hline $\begin{array}{l}\text { C. sp.A } \\
\text { C. sp. B } \\
\text { C. sp.C } \\
\text { C. sp.D }\end{array}$ & $\begin{array}{l}7 \\
8\end{array}$ & $\begin{array}{r}17 \\
4\end{array}$ & $\begin{array}{r}19 \\
2\end{array}$ & $\begin{array}{r}22 \\
7\end{array}$ & $\begin{array}{r}22 \\
4\end{array}$ & $\begin{array}{r}15 \\
4\end{array}$ & 22 & 20 & $\begin{array}{r}18 \\
2\end{array}$ & $\begin{array}{r}14 \\
3\end{array}$ & $\begin{array}{r}11 \\
1 \\
1\end{array}$ & $\begin{array}{l}7 \\
1\end{array}$ & $\begin{array}{r}10 \\
3 \\
3\end{array}$ & $\begin{array}{r}16 \\
3\end{array}$ & $\begin{array}{r}10 \\
2\end{array}$ & 16 & $\begin{array}{r}12 \\
1 \\
2\end{array}$ & $\begin{array}{r}17 \\
2\end{array}$ & $\begin{array}{l}5 \\
2\end{array}$ & $\begin{array}{r}23 \\
6\end{array}$ & $\begin{array}{r}23 \\
1\end{array}$ & $\begin{array}{r}23 \\
3\end{array}$ \\
\hline C. spp. & 3 & 1 & 2 & 2 & & 2 & & & & & & 1 & 1 & 1 & 1 & 1 & 1 & & 1 & 2 & 1 & 1 \\
\hline $\begin{array}{l}\text { Globocassidulina subglobosa } \\
\text { G. ornata } \\
\text { Cassidulinoides spp. } \\
\text { Ehrenbergina hystrix } \\
\text { E. trigona }\end{array}$ & 8 & 20 & 20 & 33 & 10 & 8 & 3 & 2 & 4 & 4 & 12 & 9 & 6 & 9 & 8 & $\begin{array}{l}6 \\
1\end{array}$ & $\begin{array}{l}6 \\
2\end{array}$ & $\begin{array}{l}5 \\
1\end{array}$ & 4 & 4 & 2 & 3 \\
\hline $\begin{array}{l}\text { Chilostomella oolina } \\
\text { Allomorphina pacifica } \\
\text { Astrononion spp. } \\
\text { Nonionella spp. } \\
\text { Pullenia bulloides }\end{array}$ & $\begin{array}{l}3 \\
2 \\
1\end{array}$ & $\begin{array}{l}3 \\
2 \\
2 \\
4\end{array}$ & $\begin{array}{l}1 \\
1\end{array}$ & $\begin{array}{r}16 \\
2\end{array}$ & $\begin{array}{l}1 \\
1\end{array}$ & $\begin{array}{l}1 \\
1\end{array}$ & 4 & $\begin{array}{l}5 \\
3\end{array}$ & $\begin{array}{l}6 \\
1 \\
3\end{array}$ & $\begin{array}{l}1 \\
3\end{array}$ & 10 & $\begin{array}{l}2 \\
1\end{array}$ & 6 & 2 & $\begin{array}{l}2 \\
2\end{array}$ & $\begin{array}{l}4 \\
1 \\
1\end{array}$ & 4 & $\begin{array}{l}4 \\
2\end{array}$ & $\begin{array}{l}4 \\
1\end{array}$ & 5 & $\begin{array}{l}1 \\
1\end{array}$ & $\begin{array}{l}1 \\
4 \\
2\end{array}$ \\
\hline $\begin{array}{l}\text { P. asoloensis } \\
\text { P. quinqueloba } \\
P . \text { sp.A } \\
P . \text { sp. B } \\
P . \text { spp. }\end{array}$ & & 2 & $\begin{array}{l}1 \\
2\end{array}$ & 7 & 4 & 1 & 1 & $i$ & & & 3 & 2 & 1 & & 3 & & $\begin{array}{l}1 \\
1 \\
1\end{array}$ & 2 & 1 & 1 & 1 & $\begin{array}{l}3 \\
1\end{array}$ \\
\hline $\begin{array}{l}\text { Gyroidina acuta } \\
\text { G. broeckhiana } \\
\text { G. lamarckiana } \\
\text { G. neosoldanii }\end{array}$ & & $\begin{array}{l}1 \\
1\end{array}$ & 1 & 1 & 2 & & 1 & 1 & 1 & 1 & & & 1 & 1 & $\begin{array}{l}3 \\
1\end{array}$ & 1 & 1 & $\begin{array}{l}6 \\
2\end{array}$ & $\begin{array}{l}1 \\
1\end{array}$ & 1 & & 1 \\
\hline G. ef. quinqueloba & 5 & 3 & 10 & 13 & 10 & 4 & 2 & 4 & 7 & 6 & 1 & 4 & 3 & 3 & 6 & 7 & 3 & & 5 & 2 & 7 & 2 \\
\hline $\begin{array}{l}G . \text { sp.A } \\
\text { G. sp.B } \\
G . \text { sp.C } \\
G . \text { sp.D } \\
G \text {. spp. }\end{array}$ & 3 & 2 & $\begin{array}{l}3 \\
1\end{array}$ & 2 & 2 & 3 & $\begin{array}{l}2 \\
1\end{array}$ & $\begin{array}{l}1 \\
1\end{array}$ & 4 & 1 & & $\begin{array}{l}2 \\
2\end{array}$ & & 1 & 3 & $\begin{array}{l}1 \\
1\end{array}$ & $\begin{array}{l}2 \\
3 \\
2\end{array}$ & 4 & $\begin{array}{l}1 \\
1\end{array}$ & 2 & $\begin{array}{l}1 \\
3\end{array}$ & 6 \\
\hline $\begin{array}{l}\text { Oridorsalis umbonatus } \\
\text { O. sp. } \\
\text { Osangularia culter } \\
\text { O. bengalensis } \\
\text { Anomalina sp. }\end{array}$ & 7 & 8 & $\begin{array}{l}4 \\
1\end{array}$ & $\begin{array}{l}4 \\
2\end{array}$ & $\begin{array}{l}3 \\
1\end{array}$ & $\begin{array}{l}1 \\
1\end{array}$ & 2 & 1 & 8 & 1 & 8 & $\begin{array}{l}5 \\
3\end{array}$ & $\begin{array}{l}3 \\
1\end{array}$ & 2 & $\begin{array}{l}1 \\
2\end{array}$ & $\begin{array}{l}9 \\
5\end{array}$ & $\begin{array}{l}7 \\
4\end{array}$ & 7 & $\begin{array}{l}7 \\
1\end{array}$ & 1 & $\begin{array}{r}11 \\
1\end{array}$ & 2 \\
\hline $\begin{array}{l}\text { Anomalinoides globulosus } \\
\text { A. sp.A } \\
\text { A. sp.B } \\
\text { A. sp.C } \\
\text { A. spp. }\end{array}$ & $\begin{array}{l}2 \\
1 \\
1\end{array}$ & $\begin{array}{l}3 \\
9\end{array}$ & $\begin{array}{l}2 \\
8\end{array}$ & $\begin{array}{r}11 \\
4\end{array}$ & $\begin{array}{l}2 \\
8 \\
9\end{array}$ & 3 & $\begin{array}{l}3 \\
2 \\
1\end{array}$ & $\begin{array}{l}1 \\
1 \\
8 \\
1 \\
2\end{array}$ & $\begin{array}{c}1 \\
14 \\
1\end{array}$ & $\begin{array}{l}5 \\
4\end{array}$ & $\begin{array}{l}1 \\
6 \\
3\end{array}$ & $\begin{array}{l}8 \\
3\end{array}$ & $\begin{array}{l}3 \\
3\end{array}$ & $\begin{array}{l}6 \\
1 \\
1\end{array}$ & $\begin{array}{l}5 \\
2\end{array}$ & $\begin{array}{l}5 \\
1\end{array}$ & 5 & $\begin{array}{l}5 \\
1\end{array}$ & $\begin{array}{l}1 \\
6 \\
1\end{array}$ & $\begin{array}{l}3 \\
1\end{array}$ & $\begin{array}{l}5 \\
1\end{array}$ & $\begin{array}{l}7 \\
1\end{array}$ \\
\hline $\begin{array}{l}\text { Cibicidoides bradyi } \\
\text { C. kullenbergi } \\
\text { C. labatulus } \\
\text { C. mundulus } \\
\text { C. cf. opacus }\end{array}$ & 1 & 1 & $\begin{array}{l}3 \\
4\end{array}$ & 1 & $\begin{array}{l}2 \\
1\end{array}$ & 1 & $\begin{array}{l}2 \\
1\end{array}$ & & $\begin{array}{l}2 \\
1\end{array}$ & 2 & 2 & 1 & & 1 & 3 & 1 & $\begin{array}{l}5 \\
1\end{array}$ & $\begin{array}{l}1 \\
4 \\
1 \\
1\end{array}$ & 1 & $\begin{array}{l}2 \\
1 \\
1\end{array}$ & $\begin{array}{l}4 \\
1\end{array}$ & 3 \\
\hline $\begin{array}{l}\text { C. pseudoungerianus } \\
\text { C. wuellerstorfi } \\
\text { C. } \mathrm{sp.A} \\
\text { C. } \mathrm{spp} \text {. } \\
\text { Gavelinella sp. }\end{array}$ & 1 & 2 & $\begin{array}{l}3 \\
1\end{array}$ & $\begin{array}{l}1 \\
i\end{array}$ & 1 & 1 & $\begin{array}{l}1 \\
1 \\
1\end{array}$ & $\begin{array}{l}1 \\
1 \\
3\end{array}$ & $\begin{array}{l}1 \\
3\end{array}$ & 1 & $\begin{array}{l}1 \\
2 \\
1\end{array}$ & 1 & $\begin{array}{l}1 \\
1\end{array}$ & & $\begin{array}{l}1 \\
2 \\
2\end{array}$ & $\begin{array}{l}1 \\
1\end{array}$ & $\begin{array}{l}1 \\
1\end{array}$ & 3 & $\begin{array}{l}2 \\
1 \\
1\end{array}$ & $\begin{array}{l}4 \\
1\end{array}$ & 4 & 1 \\
\hline $\begin{array}{l}\text { Hanzawaia cf. cushmani } \\
\text { Melonis barleeanum } \\
\text { M. cf. nicobarensis } \\
\text { M. sp.A } \\
\text { M. spp. }\end{array}$ & 1 & 1 & $\begin{array}{l}2 \\
1\end{array}$ & 2 & $\begin{array}{l}2 \\
1\end{array}$ & 1 & 2 & 1 & 2 & & & 1 & i & 1 & & & $\begin{array}{l}1 \\
1\end{array}$ & 1 & $\begin{array}{l}1 \\
3\end{array}$ & $\begin{array}{l}2 \\
1 \\
1\end{array}$ & 1 & 1 \\
\hline Miscellaneous benthic foraminifers & 9 & 7 & 7 & 6 & 7 & 13 & 5 & 5 & 7 & 14 & 9 & 5 & 3 & 2 & 3 & 4 & 6 & 3 & 2 & 5 & 2 & 3 \\
\hline Total number of specimens & 229 & 185 & 184 & 279 & 177 & 160 & 130 & 193 & 224 & 151 & 153 & 186 & 141 & 134 & 152 & 180 & 158 & 167 & 142 & 166 & 174 & 190 \\
\hline
\end{tabular}


Table 1. (Continued).

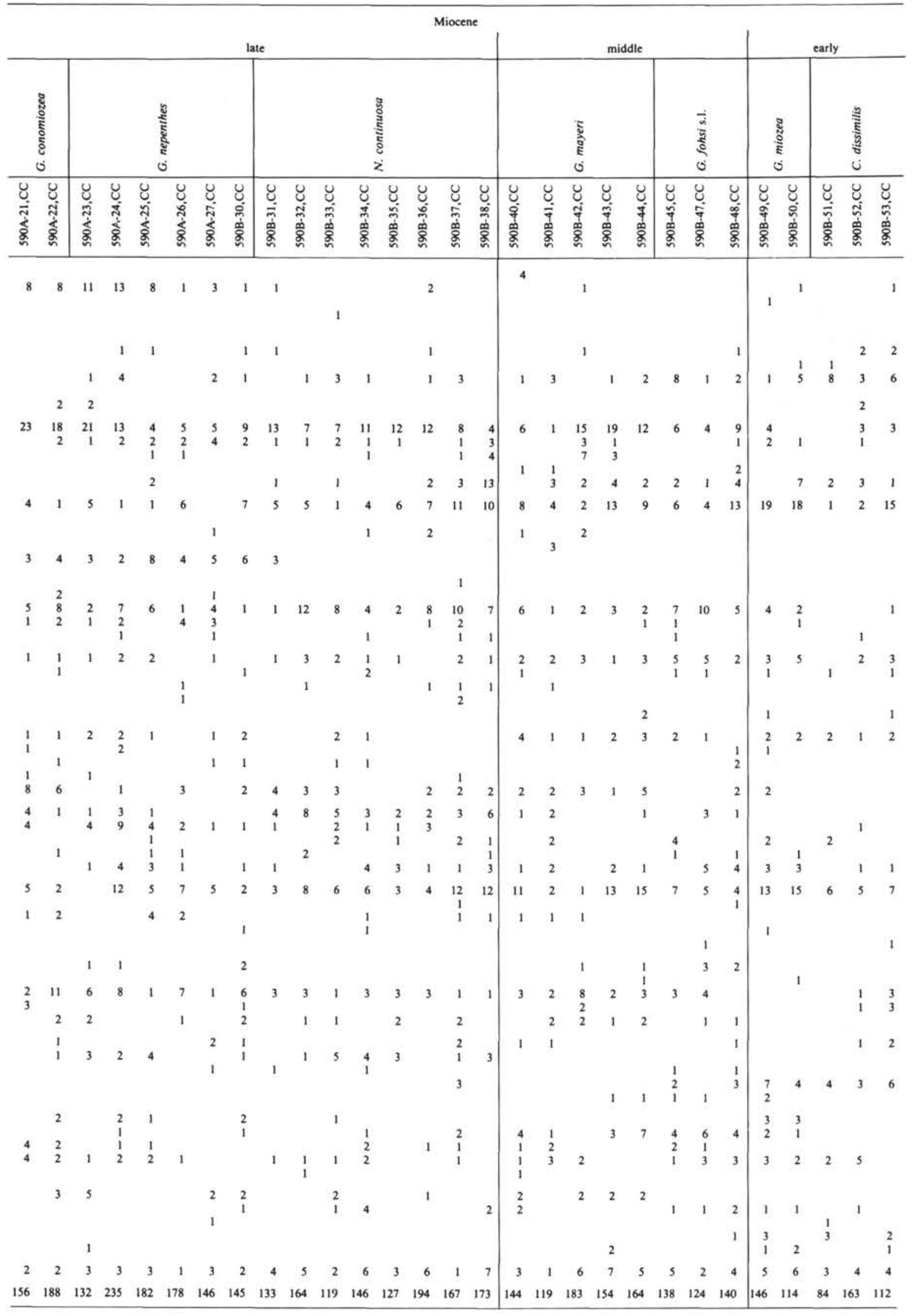


Table 2. Distribution of benthic foraminifers at Site 591.

\begin{tabular}{|c|c|c|c|c|c|c|c|c|c|c|c|c|c|c|c|c|c|c|c|c|c|c|c|c|}
\hline \multirow[b]{3}{*}{ Benthic foraminifers } & \multicolumn{3}{|c|}{ Quat. } & \multicolumn{10}{|c|}{ Pliocene } & \multicolumn{6}{|c|}{ early } & \multicolumn{5}{|c|}{$\begin{array}{c}\text { Miocene } \\
\text { late }\end{array}$} \\
\hline & \multicolumn{3}{|c|}{ 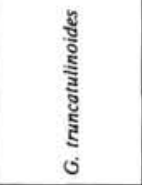 } & \multicolumn{5}{|c|}{ 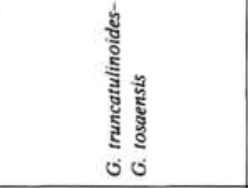 } & \multicolumn{2}{|c|}{ 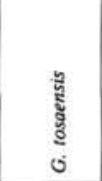 } & \multicolumn{3}{|c|}{ 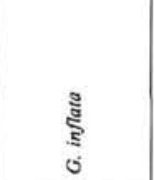 } & \multicolumn{4}{|c|}{ 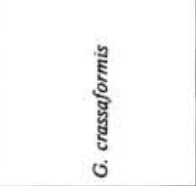 } & \multicolumn{2}{|c|}{ 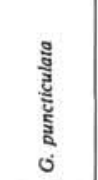 } & \multicolumn{3}{|c|}{ 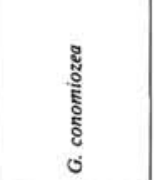 } & \multicolumn{2}{|c|}{ 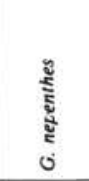 } \\
\hline & $\frac{u}{\frac{U}{a}}$ & $\begin{array}{l}\text { U } \\
\frac{1}{2}\end{array}$ & $\frac{0}{0}$ & $\begin{array}{l}0 \\
\frac{i}{2}\end{array}$ & 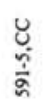 & $\begin{array}{l}\text { U } \\
\frac{0}{a} \\
\text { g }\end{array}$ & $\begin{array}{l}u \\
\frac{0}{5}\end{array}$ & 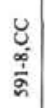 & $\begin{array}{l}u \\
\frac{0}{\alpha} \\
\frac{\alpha}{\sigma}\end{array}$ & $\begin{array}{l}\text { U } \\
\frac{0}{\dot{a}} \\
\text { a }\end{array}$ & $\frac{0}{\overline{0}}$ & 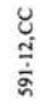 & $\frac{u}{\frac{0}{n}}$ & $\frac{u}{\frac{8}{2}}$ & $\frac{u}{0}$ & $\frac{0}{\frac{0}{1}}$ & 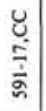 & $\begin{array}{l}u \\
\frac{0}{7} \\
\frac{\alpha}{2}\end{array}$ & $\begin{array}{l}\frac{u}{\sigma} \\
\frac{1}{\sigma}\end{array}$ & 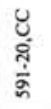 & $\begin{array}{l}\text { U্ } \\
\frac{\pi}{a}\end{array}$ & 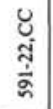 & $\begin{array}{l}\text { บु } \\
\frac{7}{\pi}\end{array}$ & 岂 \\
\hline $\begin{array}{l}\text { Bolivinopsis cubensis } \\
\text { B. sp. A } \\
\text { B. sp. B } \\
\text { Textularia lythostrota } \\
\text { T. spp. }\end{array}$ & & & & 1 & & & & & 1 & & & ${ }_{3}^{3}$ & & 1 & & 2 & 1 & $\begin{array}{l}2 \\
2\end{array}$ & $\begin{array}{l}2 \\
3\end{array}$ & 2 & & 1 & & \\
\hline $\begin{array}{l}\text { Siphotextularia rolshauseni } \\
\text { Gaudryina sp. } \\
\text { Dorothia brevis } \\
\text { Eggerella sp. A } \\
\text { E. sp. B }\end{array}$ & 2 & 2 & 1 & 1 & 3 & 2 & $\begin{array}{l}3 \\
1\end{array}$ & $\begin{array}{l}2 \\
1\end{array}$ & 1 & 5 & 2 & 1 & 5 & 2 & 1 & $\begin{array}{l}1 \\
1\end{array}$ & 3 & & $\begin{array}{l}6 \\
2\end{array}$ & 2 & 1 & $\begin{array}{l}5 \\
4 \\
1\end{array}$ & $\begin{array}{l}2 \\
2\end{array}$ & 1 \\
\hline $\begin{array}{l}\text { Karreriella bradyi } \\
\text { Martinottiella spp. } \\
\text { Ophthalmidium pusillum } \\
\text { Quinqueloculina venusta } \\
\text { Q. spp. }\end{array}$ & & $\begin{array}{l}1 \\
1\end{array}$ & 1 & 1 & & & 1 & & & & $\begin{array}{l}4 \\
1\end{array}$ & & 1 & & & 1 & 1 & & 2 & & 3 & 6 & 2 & 1 \\
\hline $\begin{array}{l}\text { Pyrgo murrhina } \\
\text { P. spp. } \\
\text { Sigmoilina edwardsi } \\
\text { S. tenuis } \\
\text { Sigmoilopsis schlumbergeri }\end{array}$ & $\begin{array}{l}2 \\
1 \\
2\end{array}$ & 2 & $\begin{array}{l}1 \\
2 \\
4 \\
2\end{array}$ & $\begin{array}{l}1 \\
2\end{array}$ & 5 & $\begin{array}{l}1 \\
1\end{array}$ & 3 & $\begin{array}{l}1 \\
6\end{array}$ & $\begin{array}{l}1 \\
2\end{array}$ & $\begin{array}{l}2 \\
3 \\
2\end{array}$ & $\begin{array}{l}2 \\
3\end{array}$ & $\begin{array}{l}1 \\
3\end{array}$ & $\begin{array}{l}1 \\
1\end{array}$ & 1 & $\begin{array}{l}2 \\
2 \\
1 \\
1\end{array}$ & $\begin{array}{l}1 \\
1 \\
1\end{array}$ & $\begin{array}{l}1 \\
2 \\
1\end{array}$ & $\begin{array}{l}2 \\
1\end{array}$ & 3 & $\begin{array}{l}2 \\
2 \\
1\end{array}$ & $\begin{array}{l}6 \\
5 \\
1\end{array}$ & $\begin{array}{l}4 \\
1\end{array}$ & $\begin{array}{l}2 \\
2 \\
1 \\
1\end{array}$ & 1 \\
\hline $\begin{array}{l}\text { Siphonaperta sp. } \\
\text { Triloculina spp. } \\
\text { Nodosaria spp. } \\
\text { Amphicoryna sp. } \\
\text { Dentalina spp. }\end{array}$ & 1 & 1 & 1 & 3 & 1 & 3 & $\begin{array}{l}1 \\
1 \\
5\end{array}$ & $\begin{array}{l}6 \\
1 \\
1\end{array}$ & $\begin{array}{l}1 \\
2\end{array}$ & $\begin{array}{l}2 \\
1 \\
2\end{array}$ & $\begin{array}{l}17 \\
1 \\
3\end{array}$ & $\begin{array}{l}1 \\
1 \\
3\end{array}$ & $\begin{array}{r}27 \\
2\end{array}$ & 2 & $\begin{array}{l}1 \\
1\end{array}$ & $\begin{array}{l}5 \\
1\end{array}$ & 3 & $\begin{array}{r}16 \\
1 \\
1 \\
1\end{array}$ & $\begin{array}{l}5 \\
3\end{array}$ & $\begin{array}{l}11 \\
1\end{array}$ & $\begin{array}{l}2 \\
1 \\
1\end{array}$ & $\begin{array}{l}5 \\
1 \\
5\end{array}$ & 20 & 1 \\
\hline $\begin{array}{l}\text { Lagena spp. } \\
\text { Lenticulina spp. } \\
\text { Vaginulina sp. A } \\
\text { Pyrulina spp. } \\
\text { Seabrookia earlandi }\end{array}$ & & $\begin{array}{l}1 \\
4\end{array}$ & 1 & 2 & $\begin{array}{l}2 \\
1\end{array}$ & 1 & 4 & 1 & $\begin{array}{l}3 \\
2\end{array}$ & 1 & 4 & $\begin{array}{l}1 \\
1\end{array}$ & $\begin{array}{l}5 \\
1 \\
2\end{array}$ & 1 & 4 & 4 & 1 & 3 & $\begin{array}{l}5 \\
1 \\
2\end{array}$ & $\begin{array}{l}2 \\
1\end{array}$ & $\begin{array}{l}1 \\
7\end{array}$ & 1 & 1 & 2 \\
\hline $\begin{array}{l}\text { Oolina spp. } \\
\text { Fissurina spp. } \\
\text { Panafissurina spp. } \\
\text { Buliminella sp. } \\
\text { Sphaeroidina bulloides }\end{array}$ & $\begin{array}{l}3 \\
5\end{array}$ & $\begin{array}{l}4 \\
2\end{array}$ & $\begin{array}{r}1 \\
10 \\
1 \\
2\end{array}$ & $\begin{array}{l}2 \\
6\end{array}$ & ${ }_{11}^{2}$ & $\begin{array}{l}3 \\
7\end{array}$ & $\begin{array}{l}2 \\
8\end{array}$ & $\begin{array}{l}1 \\
3 \\
2 \\
3\end{array}$ & $\begin{array}{l}2 \\
7 \\
1 \\
1\end{array}$ & $\begin{array}{l}4 \\
2\end{array}$ & $\begin{array}{l}3 \\
3 \\
1 \\
1\end{array}$ & $\begin{array}{l}2 \\
9\end{array}$ & $\begin{array}{l}3 \\
3\end{array}$ & 2 & 3 & $\begin{array}{l}1 \\
9 \\
1\end{array}$ & $\begin{array}{l}7 \\
5\end{array}$ & $\begin{array}{l}2 \\
6 \\
2\end{array}$ & $\begin{array}{l}2 \\
8 \\
3 \\
1\end{array}$ & $\begin{array}{l}3 \\
5\end{array}$ & $\begin{array}{r}3 \\
11\end{array}$ & 5 & $\begin{array}{l}3 \\
7 \\
1 \\
2\end{array}$ & $\begin{array}{l}2 \\
3\end{array}$ \\
\hline $\begin{array}{l}\text { Bolivina anastomosa } \\
\text { B. decussata } \\
\text { B. minuta } \\
\text { B. pusilla } \\
\text { B. seminuda }\end{array}$ & $\begin{array}{l}2 \\
2 \\
1 \\
5\end{array}$ & $\begin{array}{l}1 \\
7 \\
7\end{array}$ & 2 & 4 & $\begin{array}{l}4 \\
1\end{array}$ & $\begin{array}{l}8 \\
2 \\
6\end{array}$ & $\begin{array}{l}1 \\
3 \\
7\end{array}$ & $\begin{array}{l}6 \\
1\end{array}$ & $\begin{array}{l}5 \\
2\end{array}$ & $\begin{array}{l}7 \\
2 \\
1\end{array}$ & $\begin{array}{l}4 \\
3 \\
3\end{array}$ & $\begin{array}{l}8 \\
5\end{array}$ & 1 & $\begin{array}{l}2 \\
4\end{array}$ & $\begin{array}{l}6 \\
3\end{array}$ & $\begin{array}{l}8 \\
3\end{array}$ & $\begin{array}{l}4 \\
6\end{array}$ & 5 & $\begin{array}{l}7 \\
1 \\
1\end{array}$ & $\begin{array}{l}3 \\
1\end{array}$ & $\begin{array}{l}8 \\
2\end{array}$ & $\begin{array}{l}3 \\
5\end{array}$ & $\begin{array}{r}28 \\
10 \\
1\end{array}$ & $\begin{array}{l}7 \\
3 \\
2\end{array}$ \\
\hline $\begin{array}{l}\text { B. sp. A } \\
\text { B. sp. B } \\
\text { B. spp. } \\
\text { Stilostomella spp. } \\
\text { Bulimina miolaevis }\end{array}$ & & & 7 & 5 & 7 & 8 & 1 & 8 & 5 & $\begin{array}{l}1 \\
5\end{array}$ & $\begin{array}{l}2 \\
2\end{array}$ & 10 & $\begin{array}{r}3 \\
22 \\
10\end{array}$ & 15 & 4 & $\begin{array}{l}1 \\
3 \\
5\end{array}$ & 11 & $\begin{array}{l}5 \\
5 \\
2\end{array}$ & 5 & 2 & 1 & 6 & 2 & 2 \\
\hline $\begin{array}{l}\text { B. rostrata } \\
\text { B. striata } \\
\text { B. spp. } \\
\text { Stainforthia complanata } \\
\text { Virgulopsis sp. }\end{array}$ & $\begin{array}{l}6 \\
1 \\
2\end{array}$ & 6 & 4 & $\begin{array}{l}1 \\
1 \\
4 \\
9\end{array}$ & $\begin{array}{l}3 \\
7\end{array}$ & $\frac{1}{7}$ & $\begin{array}{l}2 \\
7\end{array}$ & 2 & $\begin{array}{l}4 \\
2 \\
1 \\
7\end{array}$ & $\begin{array}{l}1 \\
1\end{array}$ & $\begin{array}{l}6 \\
7\end{array}$ & $\begin{array}{r}9 \\
1 \\
4 \\
10\end{array}$ & $\begin{array}{r}12 \\
3\end{array}$ & $\begin{array}{l}6 \\
1 \\
2 \\
1\end{array}$ & $\begin{array}{l}1 \\
1\end{array}$ & $\begin{array}{l}1 \\
1 \\
1\end{array}$ & $\begin{array}{l}1 \\
2\end{array}$ & $\frac{1}{5}$ & 5 & 1 & $\frac{1}{7}$ & $\begin{array}{l}1 \\
1\end{array}$ & 5 & $\begin{array}{l}5 \\
1 \\
1 \\
5\end{array}$ \\
\hline $\begin{array}{l}\text { Uvigerina hispida } \\
U \text {. pengrina } \\
U \text {. proboscidea } \\
U \text {. vadescens } \\
U \text {. sp. A }\end{array}$ & & & & 2 & 2 & 5 & 10 & 1 & 5 & 2 & 5 & $\begin{array}{l}1 \\
7\end{array}$ & 1 & 2 & 1 & 1 & $\begin{array}{l}1 \\
2\end{array}$ & 2 & 1 & 3 & 2 & $\begin{array}{l}5 \\
2 \\
1\end{array}$ & 2 & 1 \\
\hline $\begin{array}{l}U . \text { sp. B } \\
U . \text { sp. C } \\
U . \text { sp. D } \\
U . \text { spp. } \\
\text { Rectuvigerina sp. }\end{array}$ & & & & & & & & & & & & & & & & & & & & & & & & \\
\hline $\begin{array}{l}\text { Trifarina sp. } \\
\text { Discorbis sp. } \\
\text { Discorbinella bertheloti }\end{array}$ & & & 1 & & & & & & & & & & 1 & 4 & 1 & 3 & & & 7 & 1 & 1 & 1 & 7 & 7 \\
\hline $\begin{array}{l}\text { Epistominella exigua } \\
\text { E. rotunda }\end{array}$ & $\begin{array}{l}26 \\
50\end{array}$ & $\begin{array}{l}32 \\
22\end{array}$ & $\begin{array}{r}8 \\
19\end{array}$ & 18 & $\begin{array}{l}50 \\
11\end{array}$ & $\begin{array}{l}19 \\
12\end{array}$ & $\begin{array}{l}35 \\
24\end{array}$ & $\begin{array}{l}17 \\
13\end{array}$ & $\begin{array}{l}11 \\
16\end{array}$ & $\begin{array}{l}42 \\
25\end{array}$ & $\begin{array}{l}36 \\
10\end{array}$ & $\begin{array}{r}26 \\
8\end{array}$ & $\begin{array}{l}27 \\
14\end{array}$ & $\begin{array}{r}20 \\
9\end{array}$ & $\begin{array}{l}20 \\
12\end{array}$ & $\begin{array}{l}21 \\
11\end{array}$ & $\begin{array}{r}41 \\
9\end{array}$ & $\begin{array}{l}8 \\
9\end{array}$ & $\begin{array}{r}18 \\
7\end{array}$ & $\begin{array}{l}39 \\
12\end{array}$ & $\begin{array}{r}25 \\
1\end{array}$ & $\begin{array}{r}17 \\
3\end{array}$ & $\begin{array}{r}16 \\
6\end{array}$ & $\begin{array}{r}23 \\
8\end{array}$ \\
\hline $\begin{array}{l}\text { E. rugasa } \\
\text { E. umbonifera } \\
\text { E. sp. } \\
\text { Gavelinopsis lobatulus } \\
\text { Laticarinina alto-camerata }\end{array}$ & 4 & 4 & $\begin{array}{l}8 \\
1\end{array}$ & 1 & 1 & & 1 & $\begin{array}{l}1 \\
2\end{array}$ & 2 & 4 & 6 & 2 & 5 & 1 & 2 & 11 & $\begin{array}{l}6 \\
1\end{array}$ & $\begin{array}{l}5 \\
1\end{array}$ & 4 & 13 & $\begin{array}{l}2 \\
2\end{array}$ & 1 & & $\frac{1}{2}$ \\
\hline
\end{tabular}

Note: Number represents no. specimens counted in $>63 \mu \mathrm{m}$. Planktonic foraminifer zones and DSDP sample nos, in column heads. 


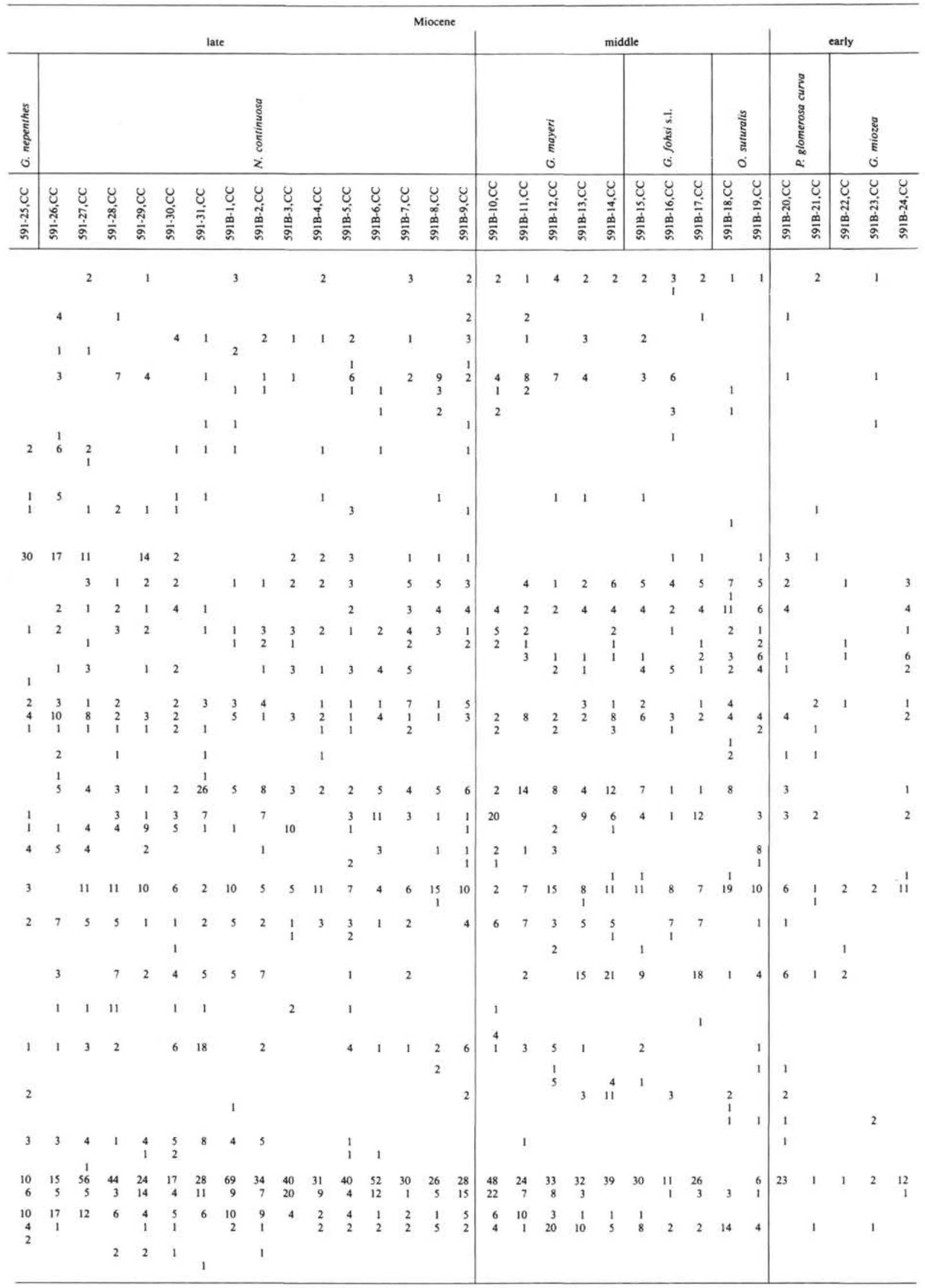


Table 2. (Continued).

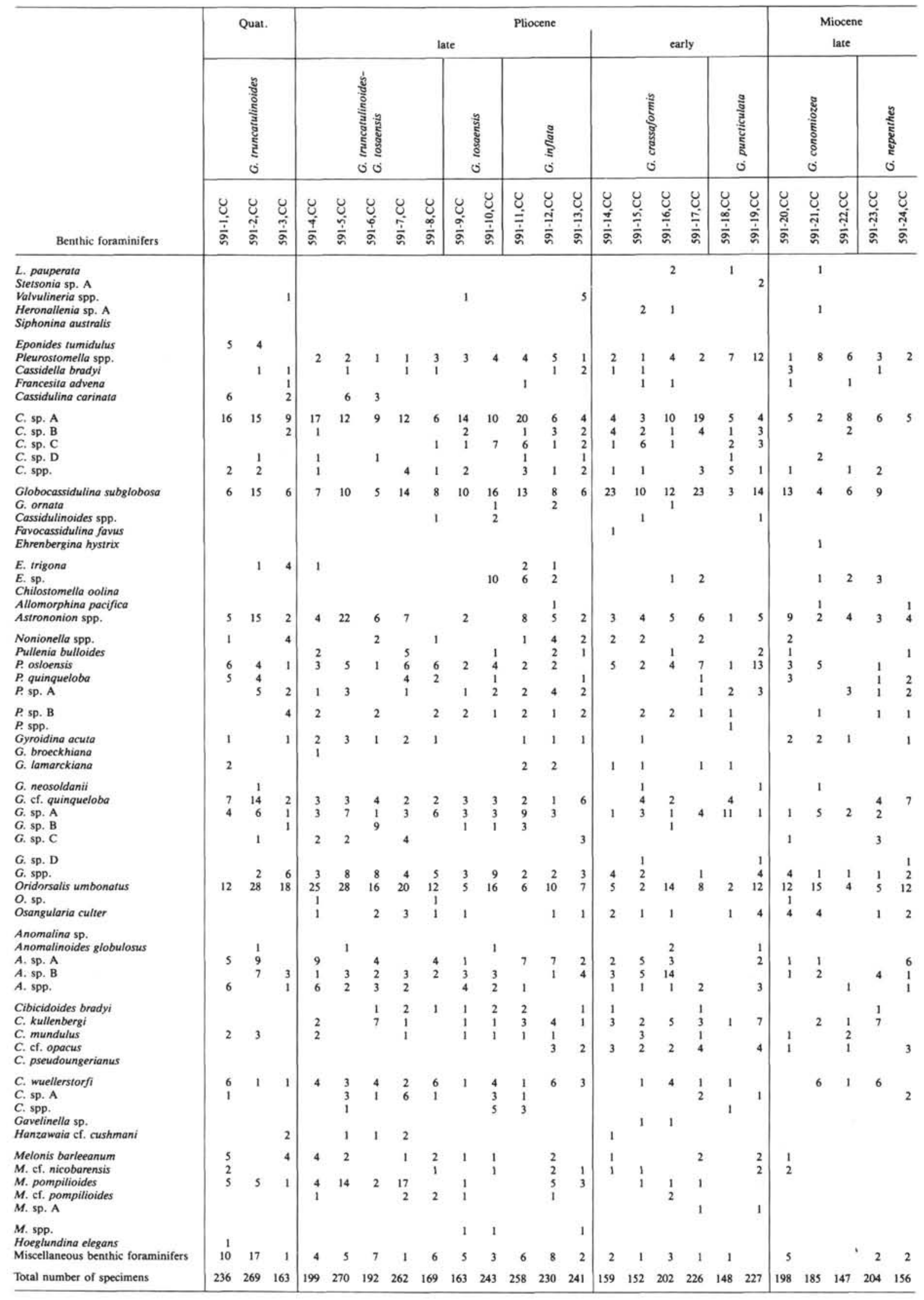


Table 2. (Continued).

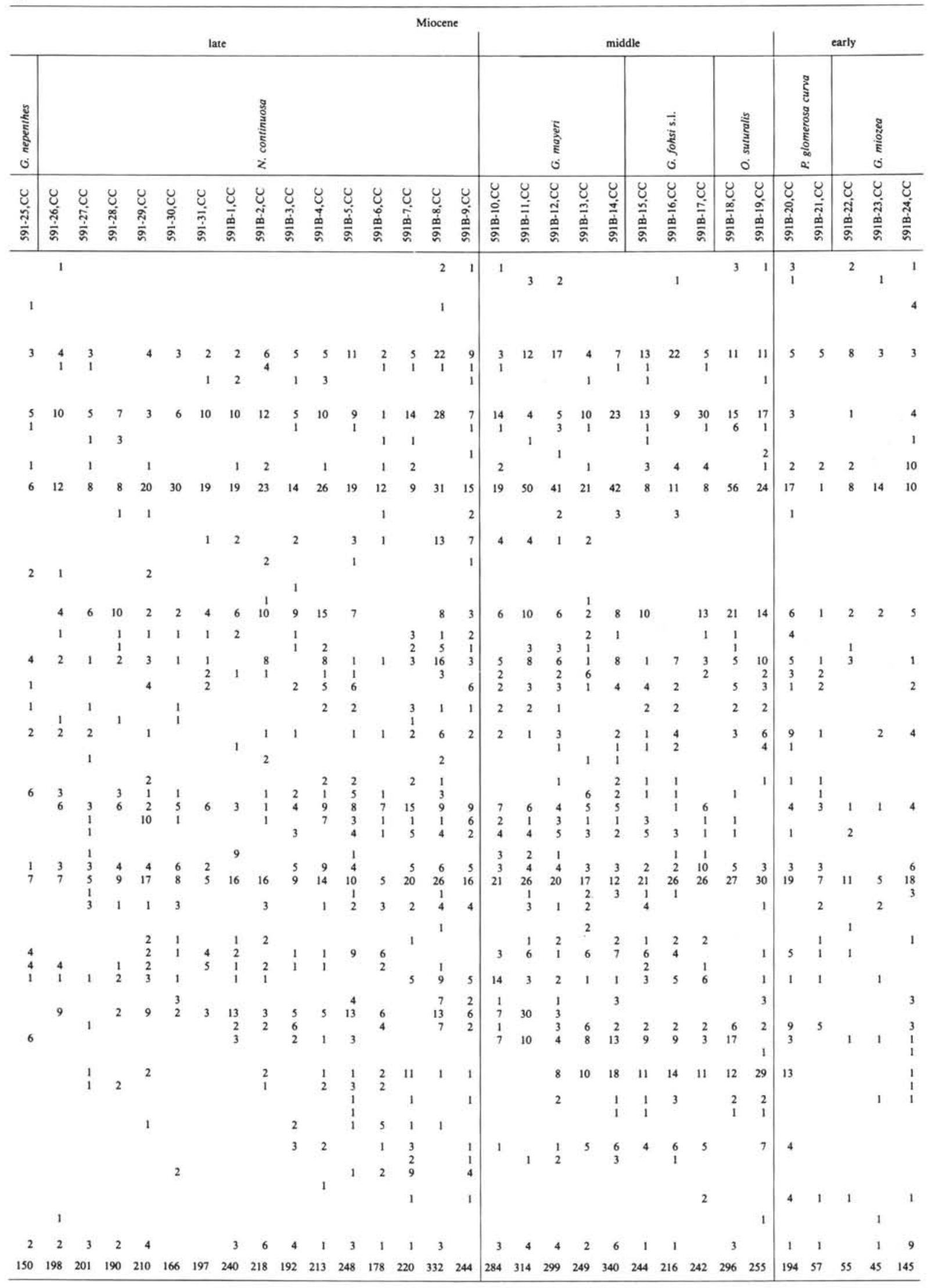


ous faunas are contained throughout the Neogene section. P/B ratios are lower than at the two shallower sites, probably because some planktonic foraminiferal tests were dissolved or broken at this greater water depth. The ratios range from 8:1 to 1864:1 (Fig. 4). In the early Miocene, the ratios are high and average value is $454: 1$. $\mathrm{P} / \mathrm{B}$ ratios are rather constant after the early Miocene and mostly average less than 100:1.

The stratigraphic distribution of benthic foraminifers is shown in Table 3. The most important species in this site include Epistominella exigua, Globocassidulina subglobosa, E. rotunda, Epistominella umbonifera (Cushman), Bolivina pusilla, B. decussata, Oridorsalis umbonatus, and Cassidulina sp. A. Almost all species at this site are also commonly found at the other two sites. Species occurring more frequently than at the other two sites are Buliminella sp., E. umbonifera, Favocassidulina favus (Brady), and Stetsonia sp. A. The following species, which occur at the other two sites and are absent from this site include Seabrookia earlandi Wright, Cibicidoides kullenbergi (Parker), and Gyroidina broeckhiana (Karrer).

Early Miocene assemblages were dominated by $G$. subglobosa and E. exigua. Other important species include Bolivina pusilla, B. decussata, Oridorsalis umbonatus, Cassidulina sp. A, and Stetsonia sp. A.

In the middle Miocene and early late Miocene (Neogloboquadrina continuosa Zone), the assemblages were also dominated by $G$. subglobosa and E. exigua. No significant change of dominant species was recognized between the early and middle Miocene assemblages at this deepest site. Other important species include O. umbonatus, Bulimina rostrata Brady, Cassidulina sp. A, and Bolivina decussata.

In the late late Miocene (Globigerina nepenthes Zone), the assemblages were dominated by $E$. umbonifera. Other important species include E. exigua, Globocassidulina subglobosa, and Cassidulina sp. C. Cassidulina sp. A almost disappeared during this interval.

Early Pliocene assemblages were again dominated by $G$. subglobosa and $E$. exigua as in the early to early late Miocene. Other important species include $E$. rotunda, Oridorsalis umbonatus, and Virgulopsis sp.

In the late Pliocene, the assemblages were dominated by $E$. rotunda and $E$. exigua. Epistominella rotunda markedly increased in abundance during this interval. Other important species include $O$. umbonatus, G. subglobosa, E. umbonifera, and Melonis pompilioides (Fichtel and Moll).

Quaternary assemblages were dominated by $E$. exig$u a$. This species slightly decreased in frequencies near the Pliocene/Pleistocene boundary. Other important species include $E$. rotunda, Cassidulina sp. A, O. umbonatus, G. subglobosa, and Virgulospsis sp. The changing species dominance of benthic foraminiferal assemblages for Site 206 is summarized in Figure 5.

\section{COMPARISON OF BENTHIC FORAMINIFERS AMONG THE THREE SITES}

The distribution of important benthic foraminiferal species among the three sites is shown in Figure 6. Among the genera occurring in the three sections, Epistominella shows highest frequencies in most part of the sections. Globocassidulina, Cassidulina, Oridorsalis, Gyroidina, and Bolivina occur frequently in the three sites. Other genera, including Cibicidoides, Uvigerina, Pullenia, and Stilostomella, rarely exceed $10 \%$ of the fauna. Bolivina was found to occur in high frequencies in the early Miocene.

Dominant species prevailing in the three sections are E. exigua, E. rotunda, and Globocassidulina subglobo$s a$, which usually occur in frequencies between 10 and $30 \%$. The average size of specimens of these three species is small $(<150 \mu \mathrm{m})$. Other dominant species, such as Oridorsalis umbonatus, E. umbonifera, and Cassidulina carinata, show a more restricted stratigraphic distribution.

In the early Miocene, G. subglobosa was the dominant element of benthic foraminiferal assemblages in all three sites. Globocassidulina subglobosa was associated with other dominant species such as $E$. exigua in the deepest site and $O$. umbonatus at the intermediate site. In the early middle Miocene, $E$. exigua migrated upslope and became dominant at the intermediate and shallowest sites (Sites 590 and 591), whereas G. subglobosa decreased in importance at these two sites. However, $G$. subglobosa remained dominant with $E$. exigua at the deepest site for much of the remainder of the Neogene. The stratigraphic range over which $E$. exigua dominated varies from site to site, but $E$. exigua maintained near constant abundance in the deepest site. This species remained the dominant form until the beginning of the Quaternary at intermediate Site 591, but was replaced as the dominant species by $E$. rotunda in the shallowest Site 590 during the late Miocene. Epistominella umbonifera was dominant during the late Miocene only at deepest Site 206. Epistominella rotunda appeared earlier as an important form at shallower Site 590 in the late Miocene and remained dominant in the Pliocene and Quaternary. This species appeared as a dominant species at the deepest Site 206 in the late Pliocene and at intermediate Site 591 in the Quaternary. Assemblages dominated by Cassidulina carinata appeared in the late Quaternary only at the shallower site.

Among the three sites, faunal successions are most similar between the two shallowest sites (Sites 590 and 591). The deepest site (Site 206) differs in composition and distribution of dominant species. Major faunal changes of benthic foraminiferal assemblages often occurred during the same time intervals among the three sites (Fig. 5). Major changes in benthic foraminiferal assemblages occurred during the middle Miocene at Sites 590 and 591; the late Miocene at Sites 590 and 206; and near the Pliocene/Pleistocene boundary at Sites 591 and 206.

\section{RESULTS OF FACTOR ANALYSIS}

\section{Site 590}

The factor analysis of the benthic foraminifers of Site 590 , which employs 5 factors, incorporates $88 \%$ of the faunal variance. Four distinct faunal assemblages are recognizable as follows (Fig. 7): 
Factor 1: This assemblage (factor) is dominated by Epistominella rotunda and Cassidulina sp. A (39\% of the faunal variance).

Factor 2: This assemblage is dominated by Globocassidulina subglobosa and Oridorsalis umbonatus $(10 \%$ of the faunal variance).

Factor 3: This assemblage is dominated by $E$. exigua ( $28 \%$ of the faunal variance).

Factor 4: This assemblage is dominated by Bolivina pusilla. Other important species include Cassidulina sp. A, Virgulopsis sp., E. rotunda, and Siphonaperta sp. ( $7 \%$ of the faunal variance).

Distribution of these four assemblages in Site 590 is summarized as follows. The early and early middle Miocene was characterized by oscillating dominance of the G. subglobosa-O. umbonatus assemblage (factor 2) and the $B$. pusilla assemblage (factor 4). During the middle middle Miocene to middle late Miocene, the $E$. exigua assemblage (factor 3 ) became dominant and the $E$. rotunda-Cassidulina sp. A assemblage (factor 1 ) increased in importance. During the middle late Miocene to Quaternary, the fauna was dominated by the $E$. rotunda- $C$. sp. A assemblage. In the Quaternary the $G$. subglobo$s a-O$. umbonatus assemblage increased once again in importance.

\section{Site 591}

Factor analysis of the benthic foraminifers at this site (5 factors; $87 \%$ of the faunal variance) shows that five distinct faunal assemblages are recognizable, as follows (Fig. 8):

Factor 1: This assemblage is dominated by Epistominella exigua ( $35 \%$ of the faunal variance).

Factor 2: This assemblage is dominated by Globocassidulina subglobosa ( $16 \%$ of the faunal variance).

Factor 3: This assemblage is dominated by $E$. rotun$d a$. Other important species are Cassidulina sp. A and Oridorsalis umbonatus ( $12 \%$ of the faunal variance).

Factor 4: This assemblage is dominated by Siphonaperta sp. Another important species is E. rugosa $(11 \%$ of the faunal variance).

Factor 5: This assemblage is dominated by $O$. umbonatus and Cibicidoides wuellerstorfi. Other important species include E. rotunda, Virgulopsis sp., and Cassidulina sp. A ( $14 \%$ of the faunal variance).

Distribution of the five assemblages in Site 591 is summarized as follows. The early Miocene to early middle Miocene was dominated by the $O$. umbonatus- $C$. wuellerstorfi assemblage (factor 5 ) and at times by the G. subglobosa assemblage (factor 2). During the early middle Miocene to middle late Miocene the fauna was dominated by the $E$. exigua assemblage (factor 1$)$. During the middle late Miocene to earliest Pliocene the Siphonaperta sp. assemblage (factor 4) increased in importance, while the $E$. exigua assemblage still remained important. During the early late Pliocene to Quaternary the $E$. rotunda assemblage (factor 3 ) became dominant.

\section{Site 206}

Factor analysis of the benthic foraminifers of Site 206 ( 5 factors; $88 \%$ of the faunal variance) shows that five distinct faunal assemblages are recognizable, as follows (Fig. 9):

Factor 1: This assemblage is dominated by Globocassidulina subglobosa. Another important species is Oridorsalis umbonatus ( $24 \%$ of the faunal variance).

Factor 2: This assemblage is dominated by Epistominella rotunda. Other important species include $O$. umbonatus, Cassidulina sp. A, and Uvigerina peregrina $(23 \%$ of the faunal variance).

Factor 3: This assemblage is dominated by E. umbonifera. Another important species is Cassidulina sp. C (12\% of the faunal variance).

Factor 4: This assemblage is dominated by $E$. exigua . Another important species is E. rotunda ( $20 \%$ of the faunal variance).

Factor 5: This assemblage is dominated by Bolivina pusilla. Other important species include $B$. decussata and $E$. exigua ( $9 \%$ of the faunal variance).

Distribution of the five assemblages in Site 206 is summarized as follows. The early early Miocene was dominated by the $G$. subglobosa assemblage (factor 1) and the $E$. exigua assemblage (factor 4), which, in turn, were replaced during the late early Miocene by the $B$. pusilla assemblage (factor 5). This, in turn, was replaced during the latest early Miocene by the $E$. exigua assemblage (factor 4). During the early middle Miocene to early Pliocene faunas were mostly dominated again by the $G$. subglobosa assemblage as well as at times by the E. umbonifera assemblage (factor 3 ), which particularly dominated during the late Miocene. During the late Pliocene, faunas were dominated by the $E$. rotunda assemblage (factor 2). The late Quaternary was again dominated by the $E$. exigua assemblage.

The distribution of the benthic foraminiferal assemblages revealed by the factor analysis is shown in Figure 10 for the three sites. The analysis shows that the most of the assemblages tend to be dominated by single, rather than multiple, species. Only about eight species can be considered to represent dominant Neogene forms at these locations and depths on the Lord Howe Rise. Other species may be persistent, but are never dominant elements in the assemblages.

Changes in the assemblages revealed by the factor analysis (Fig. 10) are generally similar to observations based on the changes in species frequencies as summarized in Figure 5. The factor analysis allows greater assemblage subdivision in the early Miocene, when there tended to be more rapid oscillations in faunal dominance.

\section{DISCUSSION}

\section{Paleoceanography}

Benthic foraminifers tend to be relatively unchanging for long intervals of time because of the relative conservatism of the deep-sea environment. Although large numbers of benthic foraminiferal species are present in deep-sea assemblages, only a few species tend to dominate the assemblages, and it is their fluctuations that mark the important changes in the assemblages through time. The frequency changes of dominant forms usually vary from 10 to $30 \%$, which is quite large compared with most other species. The changing relative dominance 
Table 3. Distribution of benthic foraminifers at Site 206.

\begin{tabular}{|c|c|c|c|c|c|c|c|c|c|c|c|c|c|c|c|c|c|c|}
\hline \multirow[b]{3}{*}{ Benthic foraminifers } & \multicolumn{9}{|c|}{ Quaternary } & \multicolumn{9}{|c|}{ Pliocene } \\
\hline & \multicolumn{9}{|c|}{ 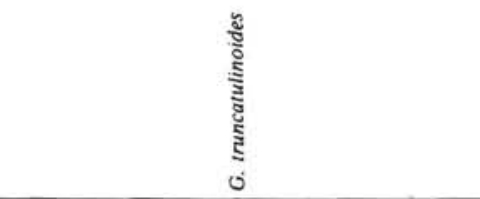 } & \multicolumn{3}{|c|}{ 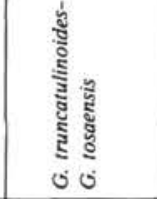 } & \multicolumn{2}{|c|}{ 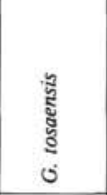 } & \multicolumn{2}{|c|}{$\begin{array}{l}\text { sั } \\
\text { ș } \\
0\end{array}$} & \multicolumn{2}{|c|}{ है } \\
\hline & 过 & 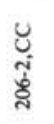 & 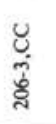 & 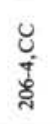 & $\begin{array}{l}\text { U } \\
\text { î. } \\
\text { ठ্ं }\end{array}$ & 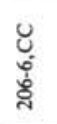 & 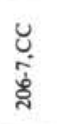 & 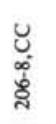 & 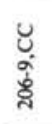 & 递 & 岂 & 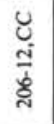 & $\underset{\tilde{m}}{\tilde{m}}$ & 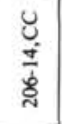 & ư & 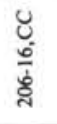 & ن & $\begin{array}{l}\ddot{U} \\
\frac{\infty}{\delta} \\
\frac{d}{\delta}\end{array}$ \\
\hline $\begin{array}{l}\text { Bolivinopsis cubensis } \\
\text { B. sp. A } \\
\text { B. sp. B } \\
\text { Vulvulina pennatula } \\
\text { Textularia spp. }\end{array}$ & 3 & 1 & & 1 & 1 & & 1 & & 1 & & 1 & & 1 & 1 & & & & 2 \\
\hline $\begin{array}{l}\text { Siphotextularia rolshauseni } \\
\text { Dorothia brevis }\end{array}$ & $\begin{array}{l}2 \\
1\end{array}$ & & & & 3 & 1 & & & 1 & 1 & 1 & & 4 & & 2 & 1 & 1 & 1 \\
\hline $\begin{array}{l}\text { Eggerella sp. A } \\
\text { K. sp. B } \\
\text { Karreriella bradyi }\end{array}$ & 4 & 1 & 2 & $\begin{array}{l}2 \\
1\end{array}$ & 1 & 2 & 6 & 3 & 5 & 2 & 7 & & 2 & $\begin{array}{l}5 \\
1\end{array}$ & 1 & 4 & $\begin{array}{r}10 \\
2\end{array}$ & 6 \\
\hline $\begin{array}{l}\text { Martinottiella spp. } \\
\text { Ophthalmidium pusillum } \\
\text { Quinqueloculina venusta } \\
\text { Q. spp. }\end{array}$ & 3 & $\begin{array}{l}1 \\
1\end{array}$ & 2 & & $\begin{array}{l}1 \\
6\end{array}$ & 4 & 1 & 1 & 2 & 2 & 1 & $\begin{array}{l}1 \\
2\end{array}$ & 1 & 1 & & 1 & $\begin{array}{l}1 \\
2\end{array}$ & \\
\hline Pyrgo murrhina & & & & & & & 5 & 1 & 1 & & 3 & & & 1 & & 1 & 2 & 1 \\
\hline $\begin{array}{l}\text { P. serrata } \\
\text { P. spp. } \\
\text { Sigmoilina edwardsi } \\
\text { S. tenuis } \\
\text { Siphonaperta sp. }\end{array}$ & & & 1 & $\begin{array}{l}1 \\
1\end{array}$ & 3 & 3 & 1 & 1 & & 1 & 1 & $\begin{array}{l}2 \\
3\end{array}$ & & 1 & $\begin{array}{l}2 \\
1\end{array}$ & 1 & 2 & 1 \\
\hline $\begin{array}{l}\text { Triloculina tricarinata } \\
\text { Nodosaria spp. } \\
\text { Astacolus sp. }\end{array}$ & 1 & & & & & & & & 1 & 1 & 2 & 1 & 3 & & & 1 & & 1 \\
\hline $\begin{array}{l}\text { Dentalina spp. } \\
\text { Lagena spp. }\end{array}$ & 4 & 1 & $\frac{1}{3}$ & $\begin{array}{l}1 \\
1\end{array}$ & $\begin{array}{l}1 \\
1\end{array}$ & 2 & & $\begin{array}{l}1 \\
1\end{array}$ & 1 & & 2 & 2 & 1 & 1 & & $\begin{array}{l}1 \\
3\end{array}$ & 1 & \\
\hline $\begin{array}{l}\text { Lenticulina spp. } \\
\text { Marginulina sp. } \\
\text { Vaginulina sp. A }\end{array}$ & & & & & & & & 1 & & & & & & 1 & 1 & 1 & & 1 \\
\hline $\begin{array}{l}\text { Pyrulina spp. } \\
\text { Oolina spp. }\end{array}$ & 1 & $\frac{1}{2}$ & & 1 & 1 & 1 & 1 & 1 & 3 & & 2 & & $\begin{array}{l}1 \\
2\end{array}$ & 2 & $\begin{array}{l}1 \\
2\end{array}$ & $\frac{1}{1}$ & 2 & ${ }_{1}^{2}$ \\
\hline $\begin{array}{l}\text { Fissurina spp. } \\
\text { Parafissurina spp. } \\
\text { Buliminella sp. }\end{array}$ & 1 & 1 & $\begin{array}{l}2 \\
2\end{array}$ & & $\begin{array}{l}3 \\
1\end{array}$ & 4 & 6 & 5 & 4 & & 3 & $\begin{array}{l}1 \\
1\end{array}$ & $\begin{array}{l}2 \\
4\end{array}$ & 3 & 2 & $\begin{array}{l}4 \\
3 \\
1\end{array}$ & 8 & 2 \\
\hline $\begin{array}{l}\text { Sphaeroidina bulloides } \\
\text { Bolivina decussata }\end{array}$ & 1 & & 2 & 3 & 1 & & 1 & & 1 & & 3 & & $\begin{array}{l}1 \\
4\end{array}$ & $\begin{array}{l}6 \\
2\end{array}$ & $\begin{array}{l}1 \\
2\end{array}$ & 1 & 6 & 1 \\
\hline $\begin{array}{l}\text { B. minuta } \\
\text { B. pusilla } \\
\text { B. cf. robusta } \\
\text { B. seminuda } \\
\text { B. sp. A }\end{array}$ & $\begin{array}{l}1 \\
1\end{array}$ & $\begin{array}{l}4 \\
1\end{array}$ & 6 & $\begin{array}{l}2 \\
1 \\
1\end{array}$ & 1 & 2 & $\begin{array}{l}5 \\
1\end{array}$ & & 5 & 2 & $\begin{array}{l}1 \\
3\end{array}$ & 5 & 1 & 2 & 10 & 4 & 8 & \\
\hline $\begin{array}{l}\text { B. sp. B } \\
\text { B. sp. D } \\
\text { B. spp. } \\
\text { Stilostomella spp. } \\
\text { Bulimina miolaevis }\end{array}$ & & & & $\begin{array}{l}4 \\
2 \\
8\end{array}$ & 1 & 1 & 1 & 1 & 7 & 1 & & 5 & & & 3 & 3 & 8 & 4 \\
\hline $\begin{array}{l}\text { B. rostrata } \\
\text { B. striata } \\
\text { B. translucens }\end{array}$ & & & & & & 1 & 2 & & & & & & & & & 1 & & \\
\hline $\begin{array}{l}\text { B. spp. } \\
\text { Stainforthia complanata }\end{array}$ & $\begin{array}{l}2 \\
3\end{array}$ & 4 & $\begin{array}{l}2 \\
2\end{array}$ & 1 & 2 & & 2 & 3 & 2 & $\frac{1}{2}$ & 1 & & 1 & 1 & & & & 3 \\
\hline $\begin{array}{l}\text { Virgulopsis sp. } \\
\text { Uvigerina peregrina } \\
\text { U. vadescens } \\
\text { U. sp. A } \\
U . \mathrm{sp} . \mathrm{B}\end{array}$ & $\begin{array}{l}6 \\
7\end{array}$ & $\begin{array}{l}6 \\
6\end{array}$ & $\frac{1}{2}$ & $\begin{array}{l}1 \\
1 \\
1\end{array}$ & $\begin{array}{l}14 \\
22\end{array}$ & $\begin{array}{r}13 \\
8\end{array}$ & $\begin{array}{r}9 \\
17\end{array}$ & $\begin{array}{r}5 \\
16\end{array}$ & $\begin{array}{r}11 \\
3\end{array}$ & $\begin{array}{l}3 \\
1\end{array}$ & 3 & $\begin{array}{l}6 \\
2\end{array}$ & $\begin{array}{l}6 \\
2 \\
5 \\
1\end{array}$ & $\begin{array}{l}3 \\
6 \\
4 \\
1\end{array}$ & $\begin{array}{l}1 \\
4 \\
3\end{array}$ & $\begin{array}{l}9 \\
2 \\
2\end{array}$ & $\begin{array}{l}9 \\
4 \\
5\end{array}$ & s \\
\hline $\begin{array}{l}\text { U. sp. C } \\
\text { U. spp. } \\
\text { Trifarina spp. }\end{array}$ & & & 1 & 7 & & & & & & & & & & & & & 1 & 1 \\
\hline $\begin{array}{l}\text { Epistominella exigua } \\
\text { E. rotunda }\end{array}$ & $\begin{array}{l}48 \\
18\end{array}$ & $\begin{array}{l}30 \\
11\end{array}$ & $\begin{array}{l}36 \\
11\end{array}$ & $\begin{array}{r}19 \\
7\end{array}$ & $\begin{array}{l}42 \\
32\end{array}$ & $\begin{array}{r}24 \\
8\end{array}$ & $\begin{array}{l}23 \\
14\end{array}$ & $\begin{array}{l}11 \\
26\end{array}$ & $\begin{array}{r}9 \\
28\end{array}$ & $\begin{array}{l}15 \\
47\end{array}$ & $\begin{array}{l}17 \\
12\end{array}$ & $\begin{array}{l}33 \\
36\end{array}$ & $\begin{array}{l}12 \\
11\end{array}$ & $\begin{array}{l}46 \\
22\end{array}$ & $\begin{array}{l}22 \\
38\end{array}$ & $\begin{array}{l}42 \\
28\end{array}$ & $\begin{array}{l}23 \\
12\end{array}$ & $\begin{array}{l}20 \\
19\end{array}$ \\
\hline $\begin{array}{l}\text { E. rugosa } \\
\text { E. umbonifera } \\
\text { E. sp. }\end{array}$ & 1 & & 1 & $s$ & 1 & & 2 & 3 & $\begin{array}{l}1 \\
2\end{array}$ & 1 & $\frac{1}{3}$ & 1 & 24 & $\begin{array}{r}1 \\
28\end{array}$ & 14 & $\frac{7}{2}$ & 13 & 5 \\
\hline
\end{tabular}

Note: Number represents no. specimens counted in $>63 \mu \mathrm{m}$. Planktonic foraminifer zones and DSDP sample nos. in column heads. 
BENTHIC FORAMINIFERS IN DEPTH TRAVERSE, SOUTHWEST PACIFIC

Table 3. (Continued).

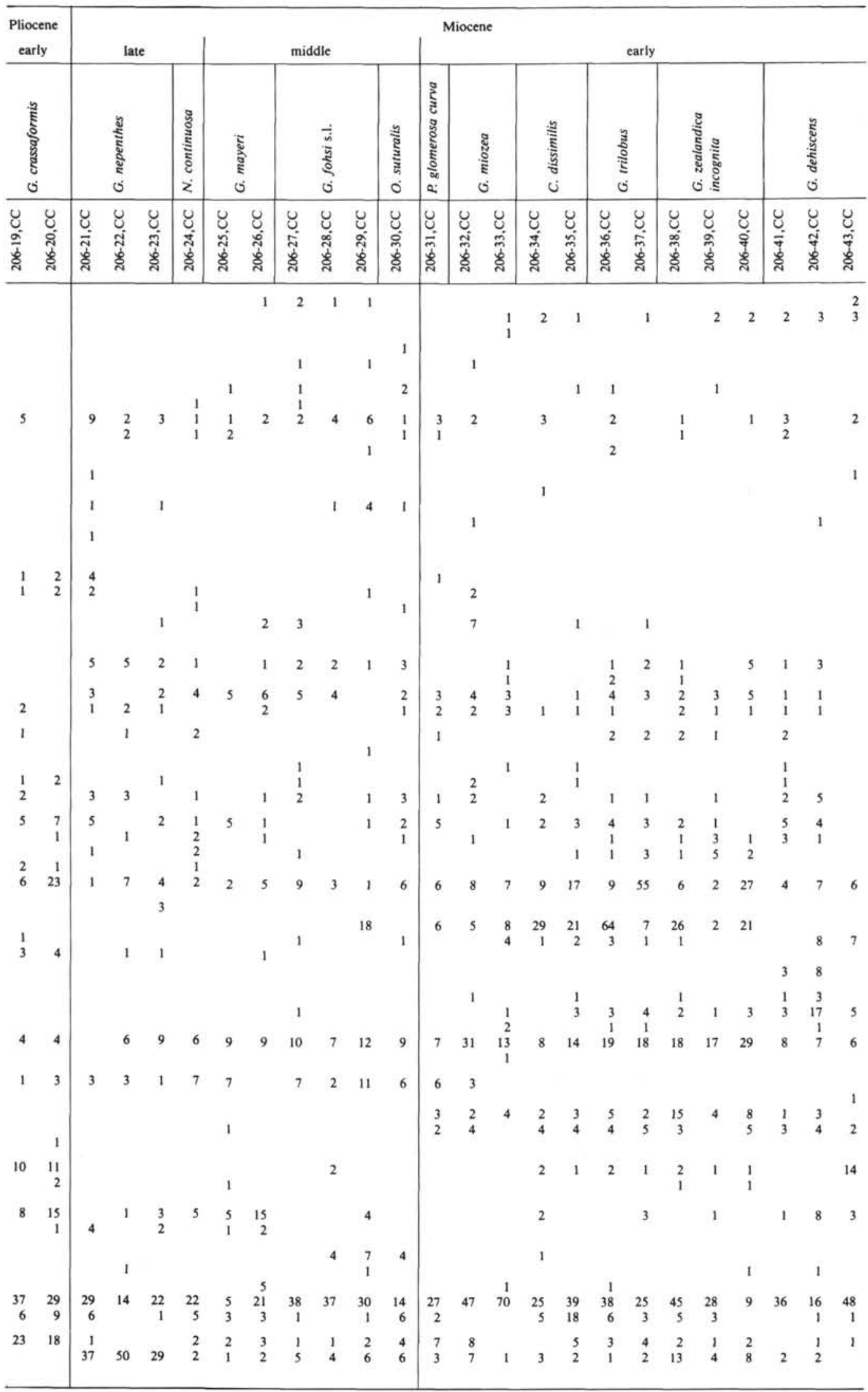


Table 3. (Continued).

\begin{tabular}{|c|c|c|c|c|c|c|c|c|c|c|c|c|c|c|c|c|c|c|}
\hline \multirow[b]{3}{*}{ Benthic foraminifers } & \multicolumn{9}{|c|}{ Quaternary } & \multicolumn{9}{|c|}{ Pliocene } \\
\hline & & & & & $\begin{array}{l}\text { ปี้ } \\
\text { हूँ } \\
\text { हूँ } \\
\text { है } \\
0\end{array}$ & & & & & & 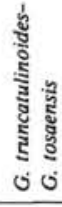 & & & : & & $\begin{array}{l}\frac{g}{5} \\
\text { s. } \\
\end{array}$ & & है \\
\hline & 导 & 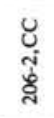 & 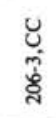 & 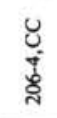 & 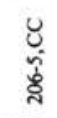 & 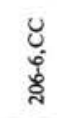 & 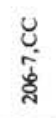 & $\begin{array}{l}0 \\
0 \\
\infty \\
\dot{d} \\
\dot{d}\end{array}$ & 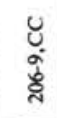 & U. & 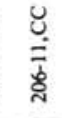 & 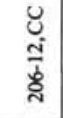 & $\begin{array}{l}\stackrel{U}{0} \\
\frac{\dot{\sigma}}{\delta} \\
\dot{d}\end{array}$ & 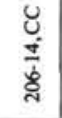 & 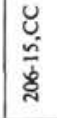 & U. & 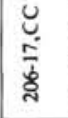 & 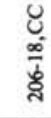 \\
\hline $\begin{array}{l}\text { Laticarinina alto-camerata } \\
\text { L. pauperata }\end{array}$ & & & & & & 1 & & & & & 3 & & & 2 & & & 1 & 1 \\
\hline $\begin{array}{l}\text { Stetsonia sp. A } \\
\text { S. sp. B } \\
\text { S. sp. C } \\
\text { Heronallenia sp. A } \\
\text { H. sp. B }\end{array}$ & & & & & & 1 & & 1 & 1 & 4 & & & 2 & & & & & $\begin{array}{l}1 \\
1\end{array}$ \\
\hline $\begin{array}{l}\text { Eponides tumidulus } \\
\text { Pleurostomella spp. }\end{array}$ & 8 & 2 & & 3 & $\begin{array}{l}1 \\
3\end{array}$ & 1 & & & $\begin{array}{l}2 \\
1\end{array}$ & & $\begin{array}{l}2 \\
1\end{array}$ & 1 & $\begin{array}{l}1 \\
6\end{array}$ & 5 & 2 & 5 & 7 & 2 \\
\hline Cassidella bradyi & 12 & 3 & 2 & & 2 & i & & & 1 & 4 & & & & & 3 & & & 1 \\
\hline $\begin{array}{l}\text { Francesita advena } \\
\text { Cassidulina carinata }\end{array}$ & & 1 & 12 & 2 & 1 & 1 & & 1 & $\begin{array}{l}2 \\
1\end{array}$ & & 1 & 3 & 1 & & 2 & 6 & $\begin{array}{l}2 \\
1\end{array}$ & \\
\hline $\begin{array}{l}\text { C. sp. A } \\
\text { C. sp. B }\end{array}$ & 15 & $\ddot{11}$ & 17 & $\begin{array}{r}26 \\
3\end{array}$ & 14 & 4 & 11 & 12 & 13 & 20 & 2 & 3 & $\begin{array}{l}2 \\
1\end{array}$ & $\begin{array}{c}10 \\
5\end{array}$ & 12 & $\begin{array}{l}5 \\
4\end{array}$ & $\begin{array}{l}6 \\
5\end{array}$ & $\begin{array}{r}12 \\
2\end{array}$ \\
\hline C. sp. C & & & & & & & & & 1 & 5 & & 4 & 8 & 7 & 6 & $\begin{array}{r}4 \\
11\end{array}$ & $\begin{array}{l}5 \\
4\end{array}$ & 2 \\
\hline C. spp. & 2 & 4 & & & 4 & 3 & 4 & 3 & 3 & 13 & & 1 & 3 & 2 & & 3 & 3 & 2 \\
\hline Globocassidulina subglobosa & 15 & 12 & 13 & 21 & 11 & 9 & 10 & 9 & 16 & 9 & 7 & 22 & 13 & 8 & 5 & 9 & 19 & 28 \\
\hline $\begin{array}{l}\text { G. ornata } \\
\text { Cassidulinoides spp. } \\
\text { Favocassidulina favus } \\
\text { Ehrenbergina hystrix } \\
\text { E. trigona }\end{array}$ & $\begin{array}{l}3 \\
2\end{array}$ & & & 1 & & & 1 & 1 & 3 & & & 1 & 1 & $\begin{array}{l}2 \\
1\end{array}$ & & 3 & 1 & $\frac{1}{1}$ \\
\hline $\begin{array}{l}\text { Chilostomella oolina } \\
\text { Allomorphina pacifica }\end{array}$ & & 2 & & & 1 & 1 & & & & & & & $\begin{array}{l}1 \\
1\end{array}$ & & & & & \\
\hline $\begin{array}{l}\text { Astrononion spp. } \\
\text { Nonionella spp. }\end{array}$ & & 1 & 1 & 3 & 6 & 3 & 2 & & $\begin{array}{l}5 \\
1\end{array}$ & 1 & 10 & 1 & $\begin{array}{l}2 \\
4\end{array}$ & & 1 & 1 & 4 & 1 \\
\hline Pullenia bulloides & & & 1 & & & & 1 & 2 & & & 1 & & 3 & 1 & $i$ & & & \\
\hline $\begin{array}{l}\text { P. osloensis } \\
\text { P. quinqueloba }\end{array}$ & 14 & 7 & 4 & & 5 & $\begin{array}{l}3 \\
2\end{array}$ & $\begin{array}{r}10 \\
3\end{array}$ & 5 & $\begin{array}{l}6 \\
1\end{array}$ & 3 & $\begin{array}{l}5 \\
2\end{array}$ & 5 & $\begin{array}{l}2 \\
3\end{array}$ & 3 & & $\begin{array}{l}4 \\
2\end{array}$ & $\begin{array}{l}4 \\
1\end{array}$ & 2 \\
\hline P. sp. A & 7 & 2 & 6 & & 29 & 9 & 9 & 11 & 3 & 6 & 5 & 8 & & 4 & 1 & 3 & 5 & 1 \\
\hline $\begin{array}{l}P \text {. sp. B } \\
P \text {. spp. }\end{array}$ & 3 & 3 & & 1 & $\begin{array}{l}1 \\
1\end{array}$ & & & $\begin{array}{l}2 \\
1\end{array}$ & 1 & & $\begin{array}{l}3 \\
1\end{array}$ & 4 & 4 & 3 & & $\begin{array}{l}2 \\
2\end{array}$ & 1 & 1 \\
\hline $\begin{array}{l}\text { Gyroidina acuta } \\
\text { G. lamarckiana } \\
\text { G. neosoldanii }\end{array}$ & & & 1 & 1 & & & & 1 & & & 2 & 1 & $\begin{array}{l}2 \\
2\end{array}$ & & & $\begin{array}{l}2 \\
1\end{array}$ & 3 & 1 \\
\hline G. cf. quinqueloba & & & & 2 & & & & & 1 & & 3 & 2 & $?$ & 11 & 5 & 2 & 7 & 5 \\
\hline G. sp. A & 1 & 1 & & 1 & 1 & 5 & 5 & 1 & 4 & & 5 & 1 & 2 & 1 & 1 & 1 & 9 & 1 \\
\hline $\begin{array}{l}\text { G. sp. B } \\
\text { G. sp. C }\end{array}$ & & & & 1 & & & 1 & & 1 & 3 & 1 & $\begin{array}{l}4 \\
4\end{array}$ & 1 & 10 & & & 4 & \\
\hline G. spp. & 3 & 5 & 9 & 2 & 9 & 4 & 1 & 8 & 4 & 6 & 8 & 9 & 3 & 16 & 4 & 10 & 1 & 5 \\
\hline $\begin{array}{l}\text { Oridorsalis umbonatus } \\
\text { O. sp. }\end{array}$ & 8 & 11 & 12 & 5 & 20 & 12 & 18 & 17 & 18 & 11 & 16 & 15 & $\begin{array}{l}6 \\
3\end{array}$ & 9 & 16 & 13 & 16 & 8 \\
\hline $\begin{array}{l}\text { Osangularia culter } \\
\text { Anomalina sp. } \\
\text { Anomalinoides globulosus }\end{array}$ & & 1 & & 1 & & 2 & & & & & & 5 & & & & & 1 & \\
\hline $\begin{array}{l}\text { A. sp. A } \\
\text { A. sp. B }\end{array}$ & 2 & $\begin{array}{l}3 \\
1\end{array}$ & $\begin{array}{l}4 \\
1\end{array}$ & 1 & 6 & 8 & $\begin{array}{l}3 \\
1\end{array}$ & & & 1 & 4 & & 1 & 1 & & & 5 & \\
\hline $\begin{array}{l}\text { A. sp. C } \\
\text { A. spp. } \\
\text { Cibicidoides bradyi } \\
\text { C. lobatulus }\end{array}$ & $\begin{array}{l}1 \\
2\end{array}$ & $\begin{array}{l}1 \\
5\end{array}$ & 10 & 3 & 1 & $\begin{array}{l}1 \\
1 \\
1\end{array}$ & $\begin{array}{l}5 \\
2 \\
1\end{array}$ & 2 & $\begin{array}{l}1 \\
1\end{array}$ & $\frac{4}{2}$ & $\begin{array}{l}1 \\
4\end{array}$ & 3 & & 4 & $\begin{array}{l}1 \\
1\end{array}$ & $\begin{array}{l}2 \\
2\end{array}$ & $\begin{array}{l}1 \\
2\end{array}$ & 2 \\
\hline C. mundulus & & & & & 1 & & 1 & 2 & & 1 & 3 & & 2 & & & 1 & 2 & 3 \\
\hline $\begin{array}{l}\text { C. cf. opacus } \\
\text { C. wuellerstorfi }\end{array}$ & 4 & & 2 & & 5 & 3 & 3 & 3 & 5 & 1 & 11 & 1 & 3 & 6 & 3 & & 7 & $\begin{array}{l}3 \\
1\end{array}$ \\
\hline $\begin{array}{l}\text { C. sp. A } \\
\text { C. spp. } \\
\text { Gavelinella sp. }\end{array}$ & $\begin{array}{l}1 \\
1\end{array}$ & & 1 & & 2 & & 2 & & & $\begin{array}{l}2 \\
3\end{array}$ & 1 & $\begin{array}{l}1 \\
2\end{array}$ & $\begin{array}{l}1 \\
2\end{array}$ & 1 & 2 & 1 & & \\
\hline $\begin{array}{l}\text { Melonis barleeanum } \\
\text { M. cf. nicobarensis }\end{array}$ & ${ }_{2}^{3}$ & & & 1 & & 4 & & 2 & 4 & & 2 & 2 & 1 & 1 & 1 & 6 & 2 & 2 \\
\hline $\begin{array}{l}\text { M. cf. nicobarensis } \\
\text { M. pompilioides }\end{array}$ & $\begin{array}{l}2 \\
7\end{array}$ & 3 & 1 & 1 & 2 & 1 & 1 & 2 & 6 & 2 & 14 & 6 & $\begin{array}{l}1 \\
9\end{array}$ & $\begin{array}{l}1 \\
4\end{array}$ & 1 & 1 & $\begin{array}{l}2 \\
2\end{array}$ & 1 \\
\hline $\begin{array}{l}\text { M. sp. A } \\
\text { M. spp. }\end{array}$ & & & & & & & 1 & & & & & & & & & & & \\
\hline $\begin{array}{l}\text { M. spp. } \\
\text { Miscellaneous benthic foraminifers }\end{array}$ & & 8 & 6 & 7 & 6 & & i & & 5 & 1 & 1 & 4 & 7 & 2 & 6 & 3 & 2 & 1 \\
\hline Total number of specimens & 226 & 163 & 180 & 159 & 272 & 156 & 197 & 168 & 200 & 182 & 186 & 215 & 204 & 262 & 184 & 230 & 250 & 173 \\
\hline
\end{tabular}


Table 3. (Continued).

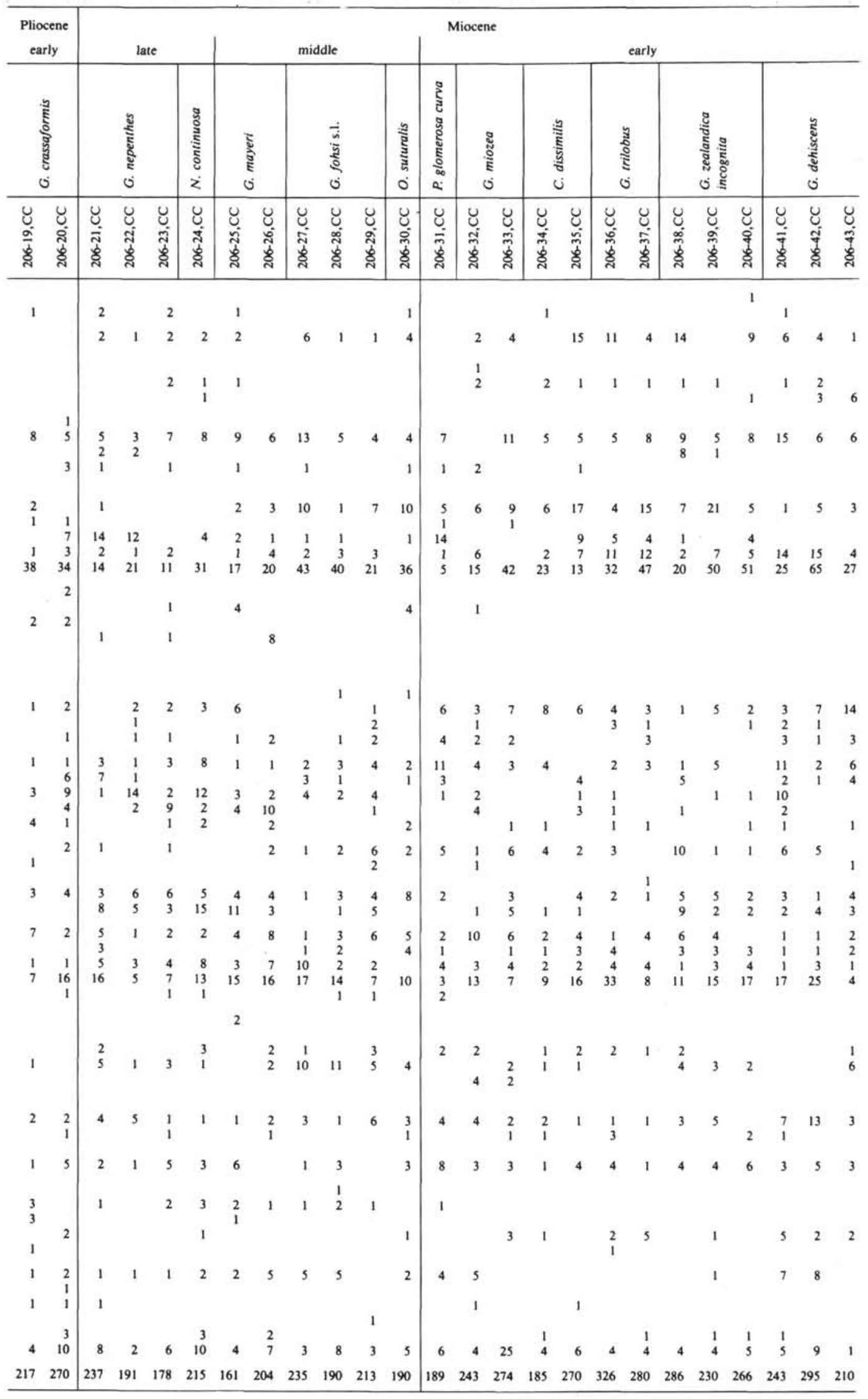




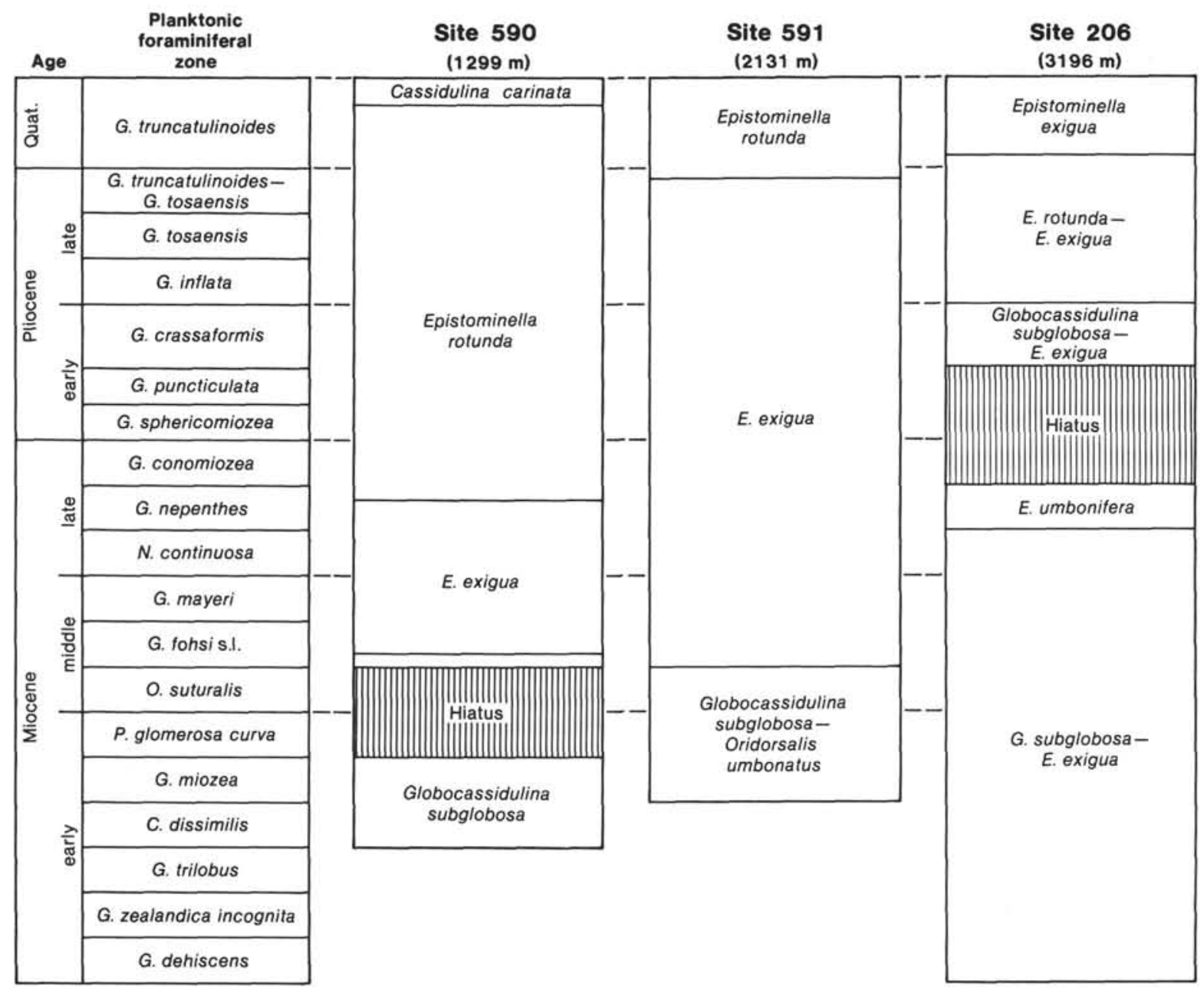

Figure 5. Dominant benthic foraminiferal species in assemblages at Sites 590, 591, and 206, as determined using changes in species frequencies (water depths in parentheses).

of a few species, particularly Epistominella exigua, E. rotunda, and Globocassidulina subglobosa, as well as E. umbonifera, Cassidulina carinata, and Oridorsalis umbonatus, define the major faunal changes that have occurred in these three sites.

Major faunal changes of benthic foraminiferal assemblages occurred in the early middle Miocene, late Miocene, and near the Pliocene/Pleistocene boundary. These faunal changes reflect global paleoceanographic events. A marked faunal change occurred in the early middle Miocene at about 14 Ma. Globocassidulina subglobosa, which prevailed as the dominant element in the early Miocene assemblages, was replaced by $E$. exigua, which had been the dominant element at the deepest Site 206 during the early Miocene. Epistominella exigua was probably associated with the water mass at the bottom of the New Caledonia Basin and migrated upslope to shallower depths during the early middle Miocene. Accordingly, dominant species of the early Miocene assemblage at the shallower two sites, namely, G. subglobosa at Site 590 and G. subglobosa and O. umbonatus at Site 591, were replaced by $E$. exigua in the early middle Miocene. This may suggest a shallowing of the deeper water masses within the New Caledonia Basin to water depths of at least $1300 \mathrm{~m}$ during the early middle Miocene. This circulation change was probably related to the development of the Antarctic ice sheet, which caused a change in the intermediate water mass structure of the southern oceans. In the three Leg 90 sites studied, high P/B ratios were recognized in the early Miocene; they decrease dramatically during the middle Miocene event (Fig. 4). In contrast, $\mathrm{P} / \mathrm{B}$ ratios at Site 289 in the equatorial $\mathrm{Pa}$ cific were shown to increase during the early middle Miocene, synchronously with the characteristic middle Miocene enrichment in $\delta^{18} \mathrm{O}$ (Woodruff and Douglas, 1981). The differences in the $\mathrm{P} / \mathrm{B}$ ratio changes probably resulted from the different size fractions studied. However, the reasons for these differences remain unresolved.

Another faunal change is found in the Globigerina nepenthes Zone of the late Miocene. By then, E. exigua prevailed as the dominant species in all three sites. However, $E$. exigua shows a decrease in frequencies during the $G$. nepenthes Zone at Site 590 , and was replaced by $E$. rotunda at this time. Synchronously with this faunal change, assemblages at Site 206 became temporarily dominated by $E$. umbonifera. This species is abundant in Cenozoic sections of deeper DSDP sites of the Pacific 
(Resig, 1976), and is associated with Antarctic Bottom Water in the Atlantic Ocean (Streeter, 1973; Schnitker, 1974) and in the Indian Ocean (Corliss, 1978). The temporary dominance of $E$. umbonifera at Site 206 may indicate the influence of deep water masses, probably Antarctic Bottom Water, during the late Miocene. Bottomwater temperatures may have cooled at the three sites during this time. This faunal change coincided with the latest Miocene carbon isotope shift (6.2 Ma) in marine sequences (Keigwin, 1979; Loutit and Kennett, 1979; and others), and with other numerous paleoceanographic changes which occurred during the latest Miocene (Kennett, 1983).

Remarkably high sedimentation rates were also found in the early Pliocene of Site 591 and were calculated to be as high as $131 \mathrm{~m} / \mathrm{m}$.y. High sedimentation rates were also recognized in the Pliocene of Site $590(57 \mathrm{~m} / \mathrm{m}$.y.). Such high rates of calcareous biogenic materials are considered to be due to high biogenic productivity. The sediments consist mostly of planktonic foraminifers and calcareous nannofossils. An increase in the $\mathrm{P} / \mathrm{B}$ ratio at this time may be expected because of the increased productivity of planktonic foraminifers. The average P/B ratio of Site 591 in the early Pliocene, during which the highest sedimentation rates were recognized, is only slightly higher than ratios in the Quaternary and the late Pliocene, and is comparable to late Miocene ratios (Table 4). The early Pliocene $\mathrm{P} / \mathrm{B}$ ratios are an order of magnitude smaller than the early Miocene ratios. P/B ratios are not significantly higher during the high sedimentation rate interval in Sites 591 and 590 . However, it is noteworthy that Site 591 , at which highest sedimentation rates were calculated, shows higher $\mathrm{P} / \mathrm{B}$ ratios than Sites 590 and 206 throughout the section. Site 591 has been marked by higher productivity of planktonic foraminifers than the other two sites since the early Miocene.

A number of interesting changes in the composition of benthic foraminiferal assemblages can be found in the latest Pliocene and early Quaternary within the Globorotalia truncatulinoides-G. tosaensis Zone and the $G$. truncatulinoides Zone. Major changes occurred in the distribution of E. exigua, which became more dominant at the deeper Site $\mathbf{2 0 6}$ and less dominant at the shallower Site 591 (Fig. 5). The data suggest that $E$. rotunda migrated to deeper water in the latest Pliocene, probably in association with an expansion of intermediate water masses. E. exigua became more restricted as an abundant form to deeper waters. Distinct surface-water cooling of the southern oceans was associated with the development of Northern Hemisphere glaciation during the late Pliocene.

Table 4. Planktonic/benthic ratios at Sites 590 and 591 .

\begin{tabular}{lcrr}
\hline \multirow{2}{*}{ Age } & \multicolumn{2}{c}{ Site 591 } & \multicolumn{1}{c}{$\begin{array}{c}\text { Site 590 } \\
\text { avg. }\end{array}$} \\
\cline { 2 - 3 } & \multicolumn{1}{c}{ P/B ratios } & Avg. & \\
\hline Quaternary & $210: 1-593: 1$ & $343: 1$ & $153: 1$ \\
late Pliocene & $179: 1-753: 1$ & $490: 1$ & $79: 1$ \\
early Pliocene & $420: 1-1662: 1$ & $756: 1$ & $141: 1$ \\
late Miocene & $151: 1-2775: 1$ & $713: 1$ & $181: 1$ \\
early Miocene & $2121: 1-10,674: 1$ & $6147: 1$ & $2522: 1$ \\
\hline
\end{tabular}

This cooling of high-latitude surface waters may have played an important role in the final development of intermediate water structure in the southwest Pacific.

\section{CONCLUSIONS}

1. All samples examined from Sites 590, 591, and 206 are dominated by relatively small individuals of benthic foraminifers in the size fractions between 63 and $150 \mu \mathrm{m}$. Foraminiferal tests smaller than $150 \mu \mathrm{m}$ make up from 50 to $99 \%$ of the assemblages and average 78 to $89 \%$ at the three sites. This demonstrates the importance of studying the small size fraction of Neogene deepsea benthic foraminifers.

2. Planktonic to benthic foraminiferal ratios (P/B ratios) were examined in the fractions larger than $63 \mu \mathrm{m}$. The ratios range approximately from $50: 1$ to $10,000: 1$. Benthic foraminifers comprise between 0.01 and $2 \%$ of the total planktonic and benthic populations. $\mathrm{P} / \mathrm{B}$ ratios indicate similar trends at the three sites. High P/B ratios occur in the early Miocene, and decrease over the early/middle Miocene boundary. The ratio remains low and almost constant with several minor peaks from the middle Miocene to the Quaternary. The decrease of the $\mathrm{P} / \mathrm{B}$ ratios at the early/middle Miocene boundary is the most remarkable change during the Neogene, and was recognized at all three sites. This faunal change is almost certainly associated with middle Miocene oceanographic changes related to increased Antarctic glaciation. Increased numbers of benthic specimens relative to planktonic forms at this time may have resulted from increased supply of organic material to the deep sea as a result of increased surface-water productivity.

3. Benthic foraminifers were studied in the fractions larger than $63 \mu \mathrm{m}$ at the three sites. The most dominant species, which prevailed in the three Neogene sections, are Epistominella exigua, E. rotunda, and Globocassidulina subglobosa. Most specimens of these three species are small $(<150 \mu \mathrm{m})$, and each usually occurs in frequencies of between 10 and $30 \%$. The assemblages are dominated by only a few forms. Single species usually dominated the assemblages at any interval of time.

4. Faunal changes of benthic foraminiferal assemblages, as shown by species frequency changes and factor analysis, occurred in the three sites through the Neogene. Faunal successions are most similar between the two shallowest sites (Sites 590 and 591). The deepest site (Site 206) has a different faunal sequence. The most important faunal changes of benthic foraminiferal assemblages occurred in the early middle Miocene (14 Ma; the Orbulina suturalis Zone and the Globorotalia fohsi s.l. Zone), in the late Miocene (6 Ma; the Globigerina nepenthes Zone), and closely associated with the Pliocene/ Pleistocene boundary (2 Ma; the Globorotalia truncatulinoides-G. tosaensis Zone and the G. truncatulinoides Zone).

5. These major faunal changes of benthic foraminiferal assemblages at the three sites are associated with known major paleoceanographic events: the middle Miocene development of the Antarctic ice sheet, the latest Miocene global cooling and increased polar glaciation, and quasiperiodic glacial oscillations of the Quaternary. 


\section{ACKNOWLEDGMENTS}

We thank D. Hodell for his assistance with the factor analysis. J. Resig and J. Ingle kindly reviewed the paper and offered useful suggestions. Financial support for K. Kurihara was provided by Rikkyo University, Tokyo. Partial support was also provided by U.S. National Science Foundation Grant OCE82-14937 (Marine Geology and Geophysics).

\section{REFERENCES}

Boltovskoy, E., 1983. Late Cenozoic deep-sea benthic foraminifera off the coast of northwest Africa (DSDP Site 369). J. African Earth Sci., 1(2):83-102.

Corliss, B. H., 1978. Studies of deep-sea benthonic foraminifera in the southeast Indian Ocean. Antarctic J. U. S., 13:116-118.

Douglas, R. G., 1973. Benthonic foraminiferal biostratigraphy in the central North Pacific, Leg 17, Deep Sea Drilling Project. In Winterer, E. L., Ewing, J. I., et al., Init. Repts. DSDP, 17: Washington (U.S. Govt. Printing Office), 607-671.
Douglas, R. G., and Woodruff, F., 1981. Deep-sea benthic foraminifera. In Emiliani, C. (Ed.), The Oceanic Lithosphere (Vol. 7): New York (John Wiley), 1233-1327.

Keigwin, L. D., Jr., 1979. Late Cenozoic stable isotope stratigraphy and paleoceanography of Deep Sea Drilling Project Sites from the East Equatorial and central North Pacific Oceans. Earth Planet. Sci. Lett., 45:361-382.

Kennett, J. P., 1973. Middle and Late Cenozoic planktonic foraminiferal biostratigraphy of the southwest Pacific-DSDP, Leg 21. In Burns, R. E., Andrews, J. E., et al., Init. Repts. DSDP, 21: Washington (U.S. Govt. Printing Office), 575-639.

1983. Paleo-oceanography: global ocean evolution. Rev. Geophys. Space Phys. 5:1258-1274.

Kennett, J. P., and Srinivasan, M. S., 1983. Neogene Planktonic Foraminifera: A Phylogenetic Atlas: Stroudsburg, PA (Hutchinson Ross).

Klovan, J. E., and Imbrie, J., 1971. An algorithm and Fortran IV for large scale Q-mode factor analysis. Int. Assoc. Math. Geol. J., 3: 61-78.

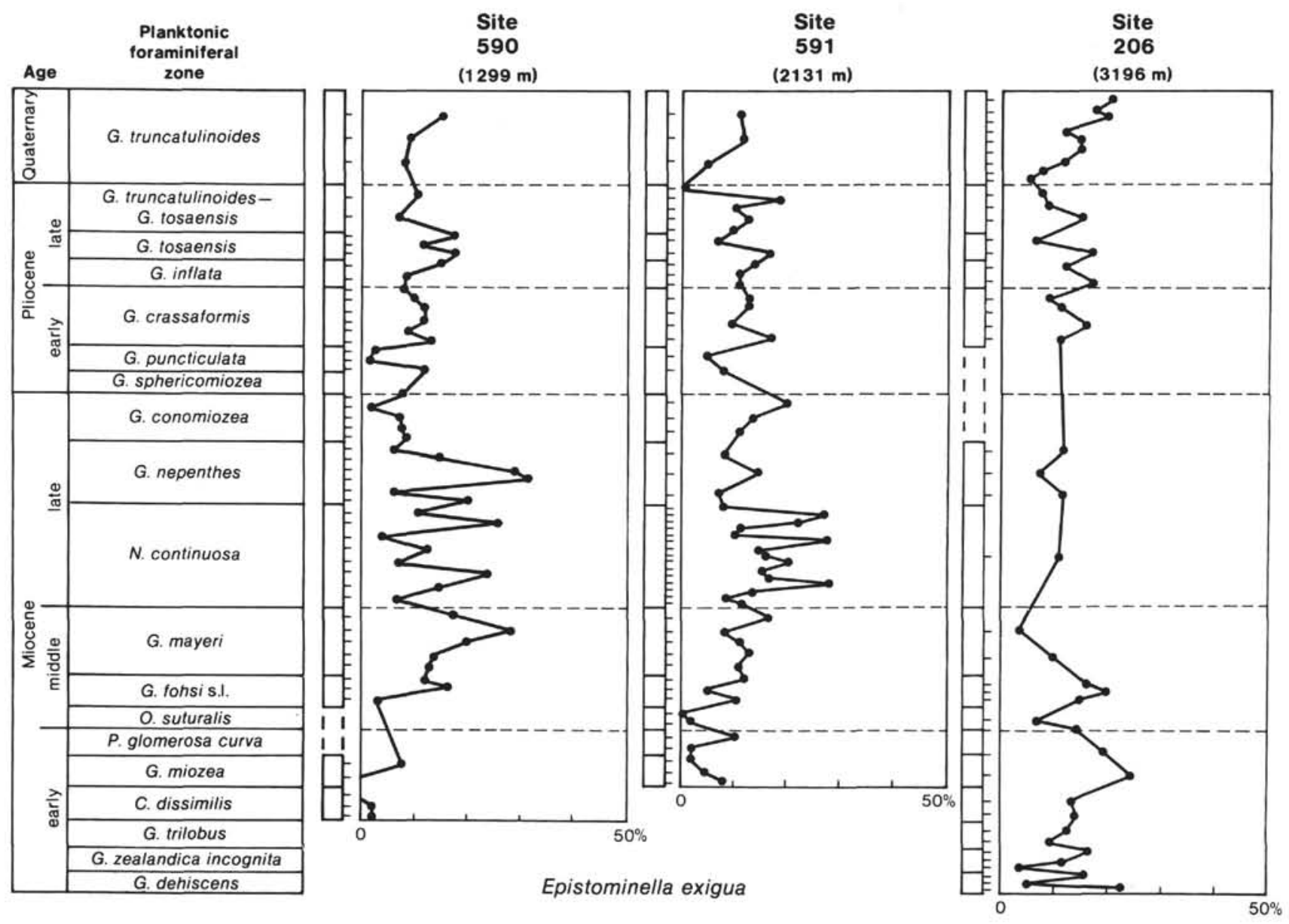

Figure 6. Distribution (species frequencies) of benthic foraminiferal species ( $>63 \mu \mathrm{m}$ ) at Sites 590, 591, and 206 (water depths in parentheses). 
Lohmann, G. P., 1978. Abyssal benthonic foraminifera as hydrographic indicators in the Western South Atlantic Ocean. J. Foram. Res., 8: 6-34.

Loutit, T., and Kennett, J. P., 1979. Application of carbon isotope stratigraphy and correlation of Late Miocene shallow marine sediments, New Zealand. Science, 204:1196-1199.

Lutze, G. F., 1979. Benthic foraminifers at Site 397: Faunal fluctuation and ranges in the Quaternary. In von Rad, U., Ryan, W. B. F., et al., Init. Repts. DSDP, 47, Pt. 1: Washington (U.S. Govt. Printing Office), 419-431.

Parker, F. L., 1958. Eastern Mediterranean foraminifera. Rept. Swedish Deep-Sea Expedition, 8(4):219-283.

Phleger, F. B., Parker, F. L., and Peirson, J. F., 1953. North Atlantic foraminifera. Rept. Swedish Deep-Sea Expedition, 7(1):3-121.

Resig, J. M., 1976. Benthic foraminiferal stratigraphy, eastern margin, Nazca Plate. In Yeats, R. S., Hart, S. R., et al., Init. Repts. DSDP, 34: Washington (U.S. Govt. Printing Office), 743-759.
Schnitker, D., 1974. West Atlantic abyssal circulation during the past 120,000 years. Nature, 248:385-387.

1979. Cenozoic deep water benthic foraminifers, Bay of Biscay. In Montadert, L., Roberts, D. G., et al., Init. Repts. DSDP, 48: Washington (U.S. Govt. Printing Office), 377-413.

Srinivasan, M. S., and Kennett, J. P., 1981. Neogene planktonic foraminiferal biostratigraphy and evolution: equatorial to Subantarctic, South Pacific. Mar. Micropaleontol., 6:499-533.

Streeter, S. S., 1973. Bottom water and benthonic foraminifera in the North Atlantic-glacial-interglacial contrasts. Quat. Res., 3: 131-141.

Woodruff, F., and Douglas, R. G., 1981. Response of deep-sea benthic foraminifera to Miocene paleoclimatic events, DSDP Site 289. Mar. Micropaleontol., 6:617-632.

Date of Initial Receipt: 17 August 1984 Date of Acceptance: 7 December 1984

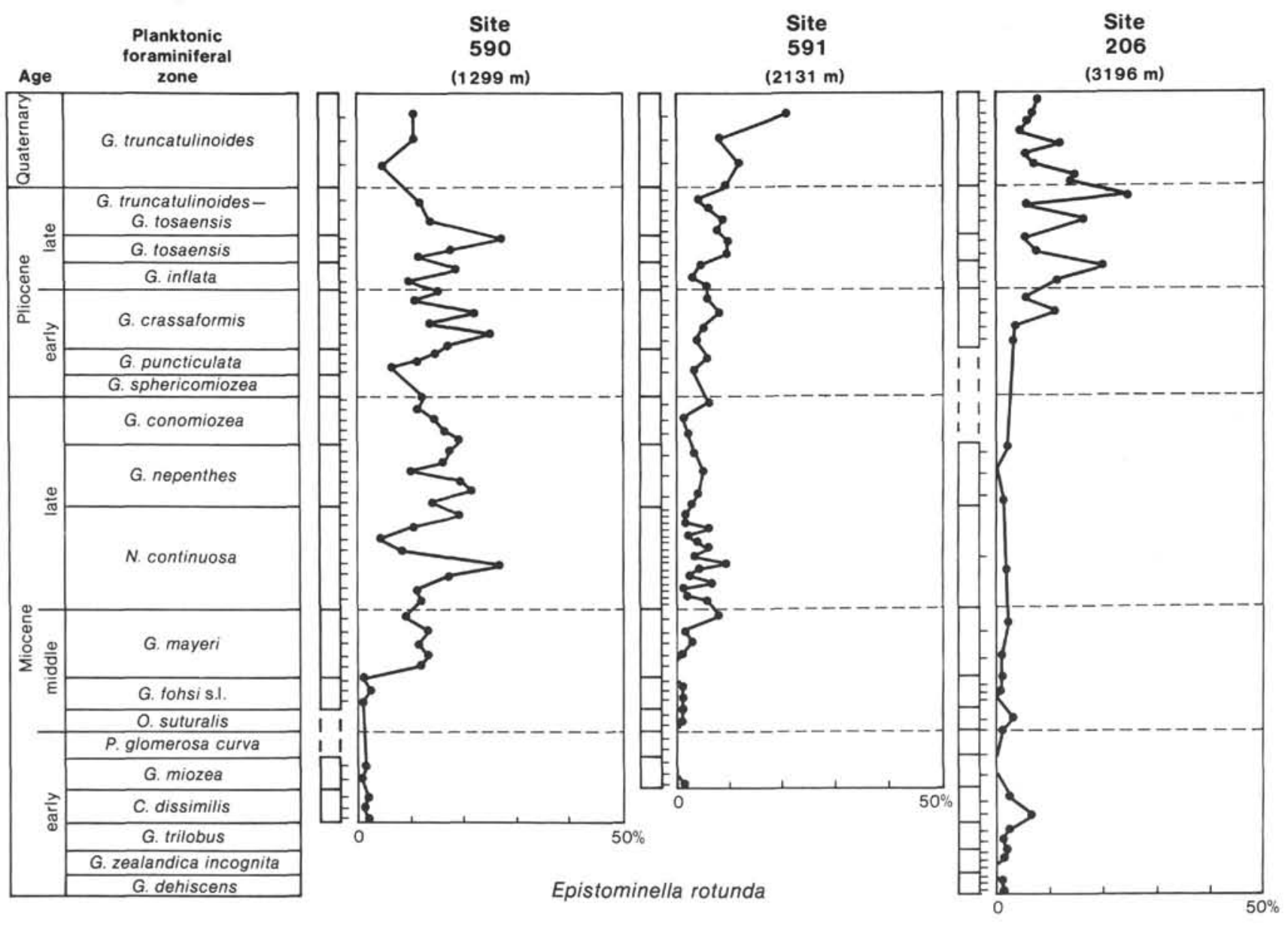

Figure 6. (Continued). 


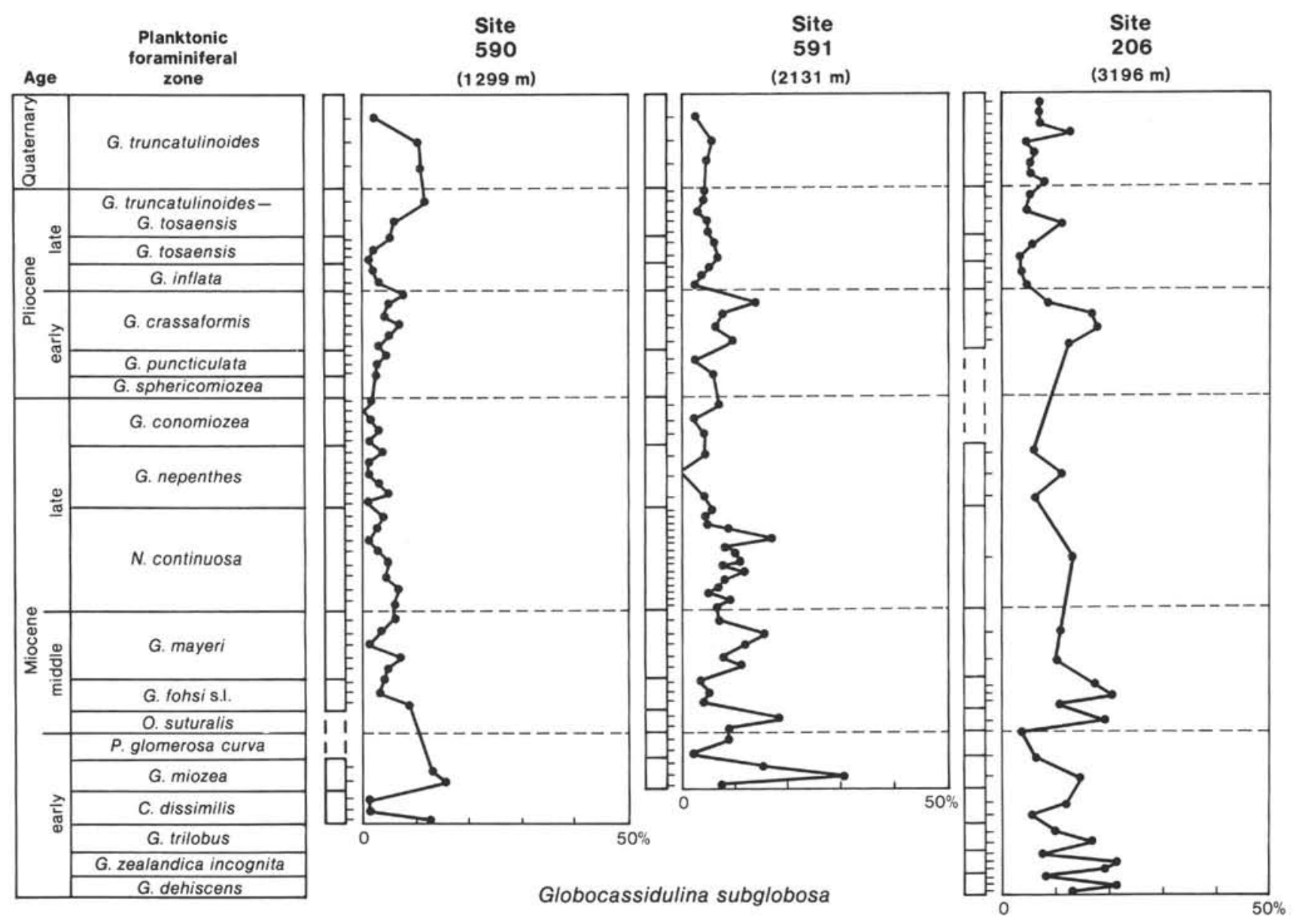

Figure 6. (Continued). 


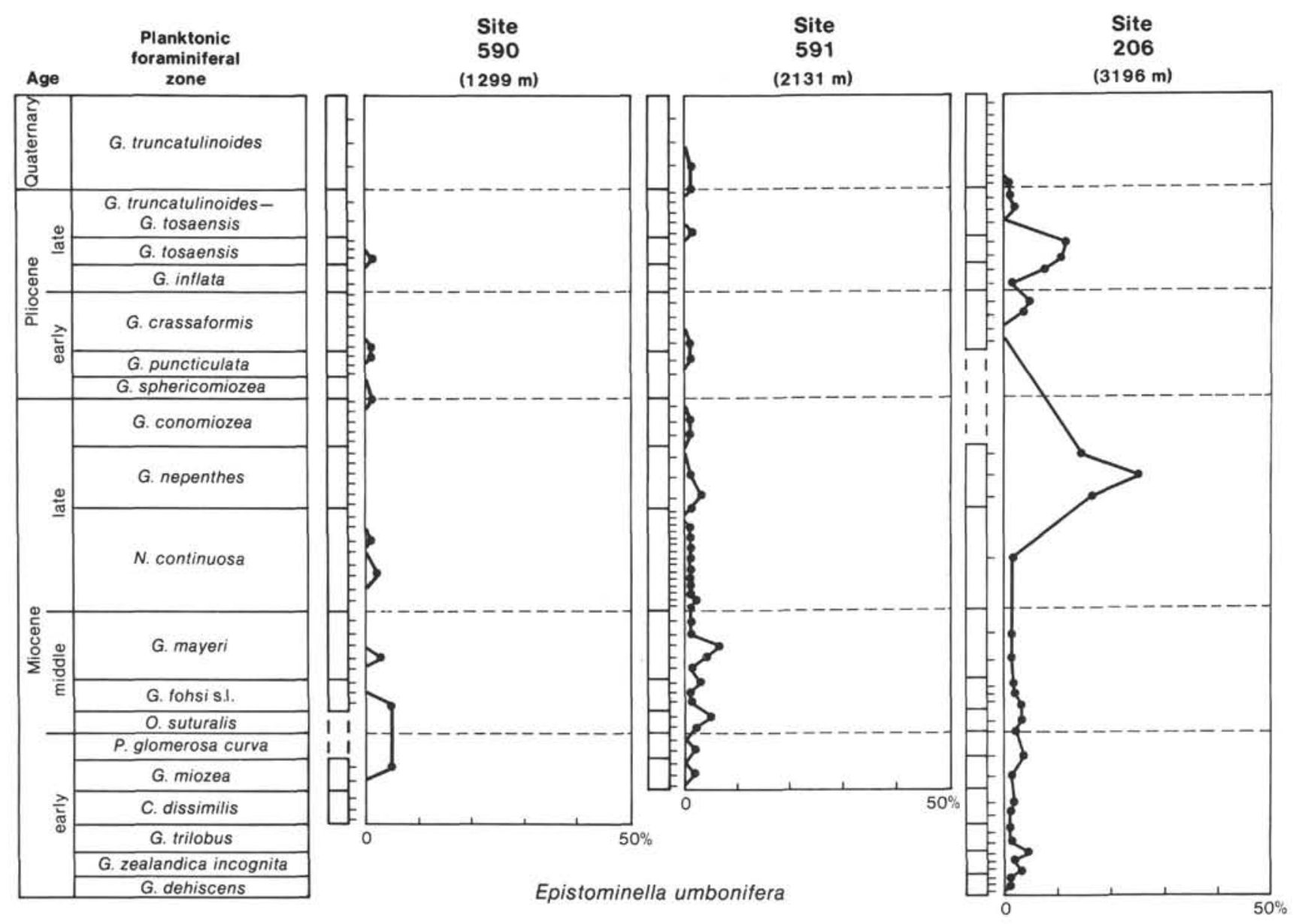

Figure 6. (Continued). 


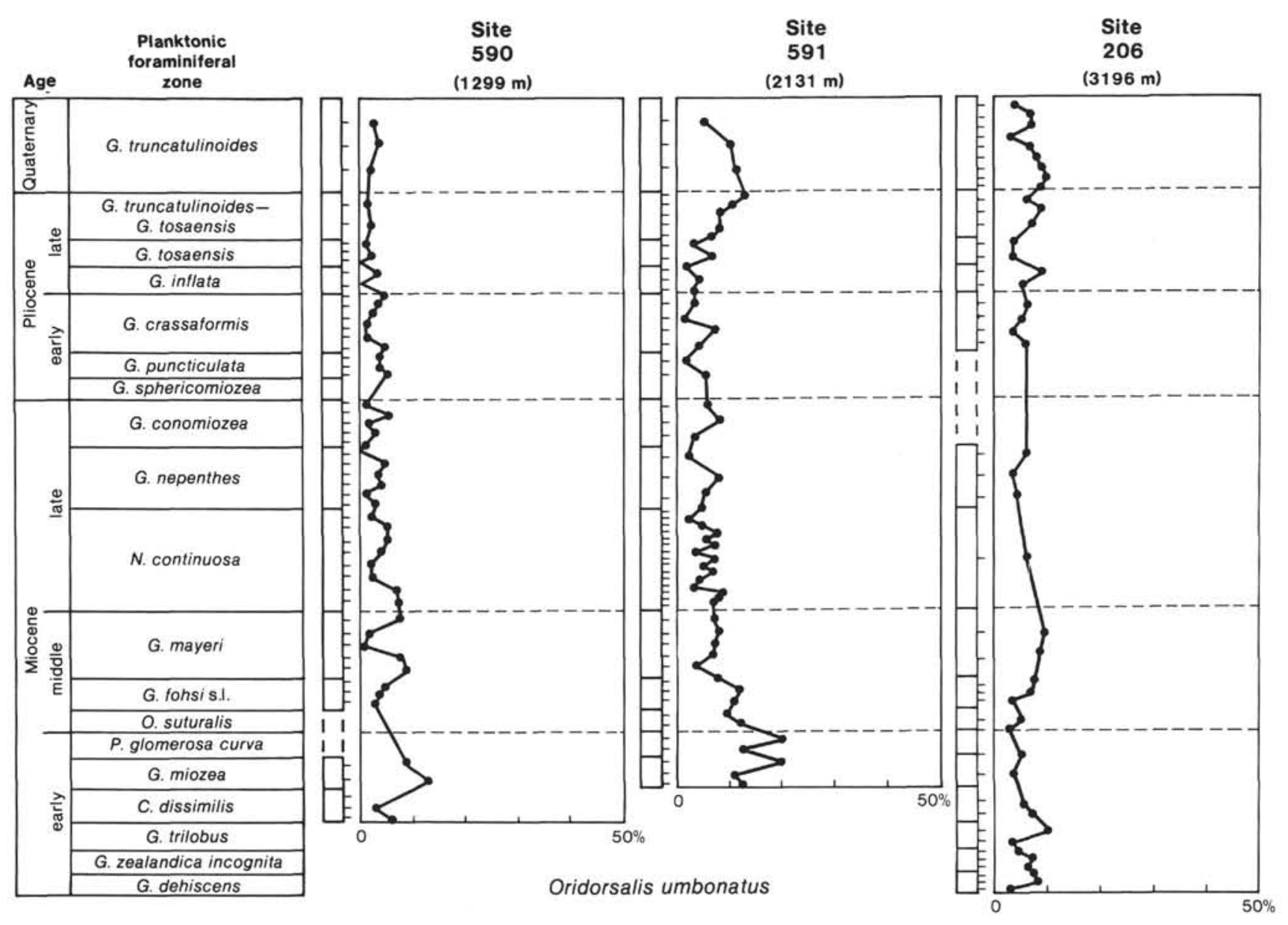

Figure 6. (Continued). 


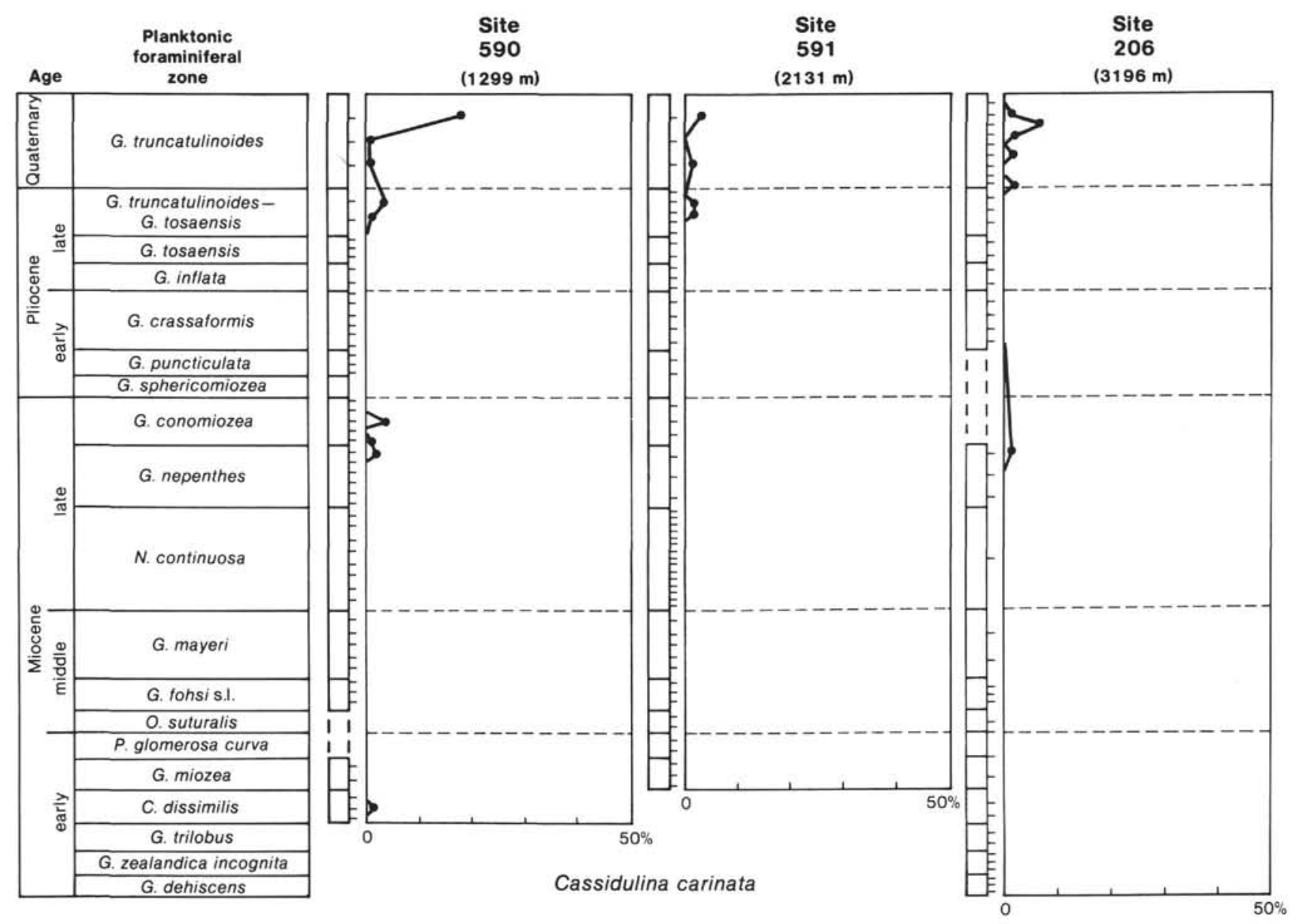

Figure 6. (Continued). 


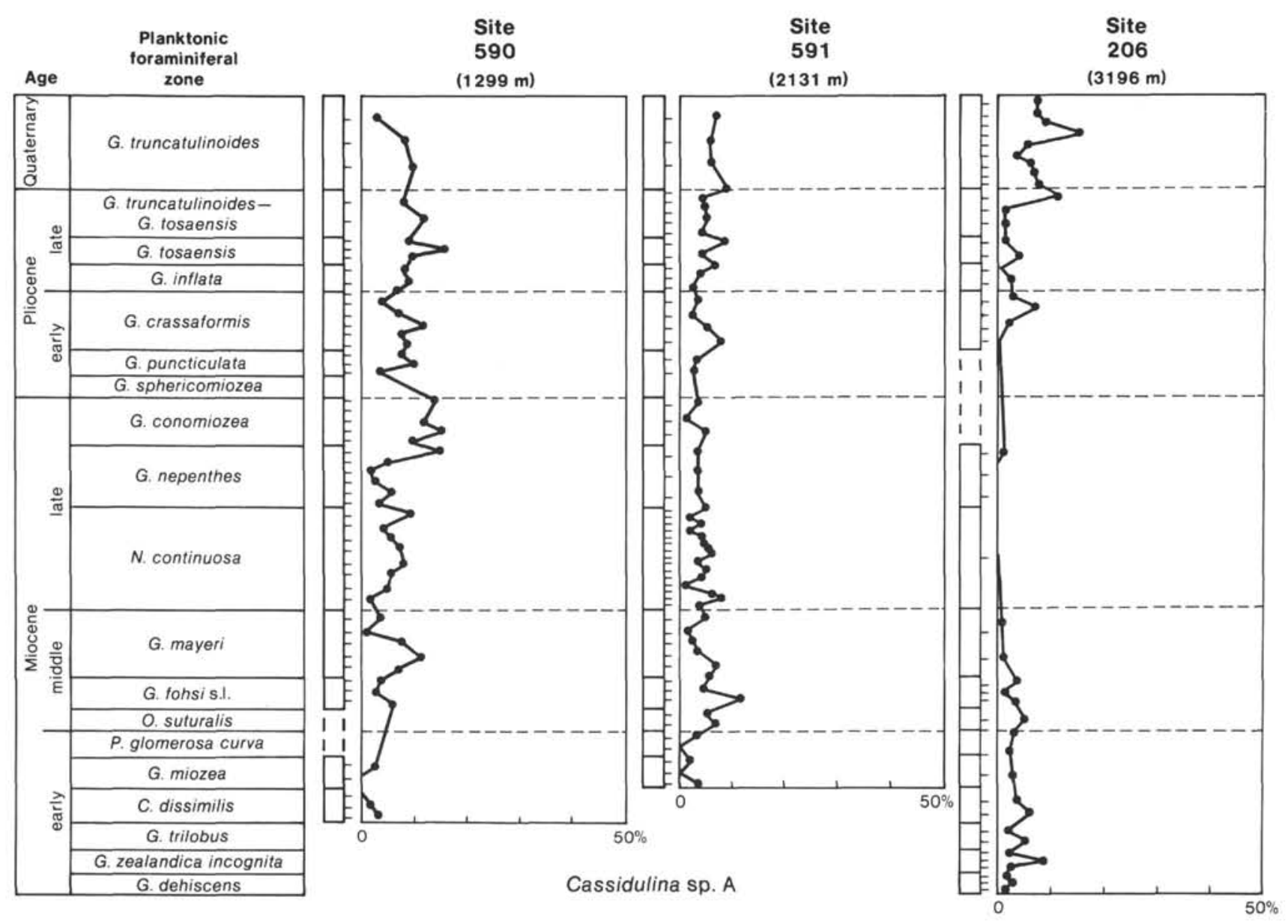

Figure 6. (Continued). 


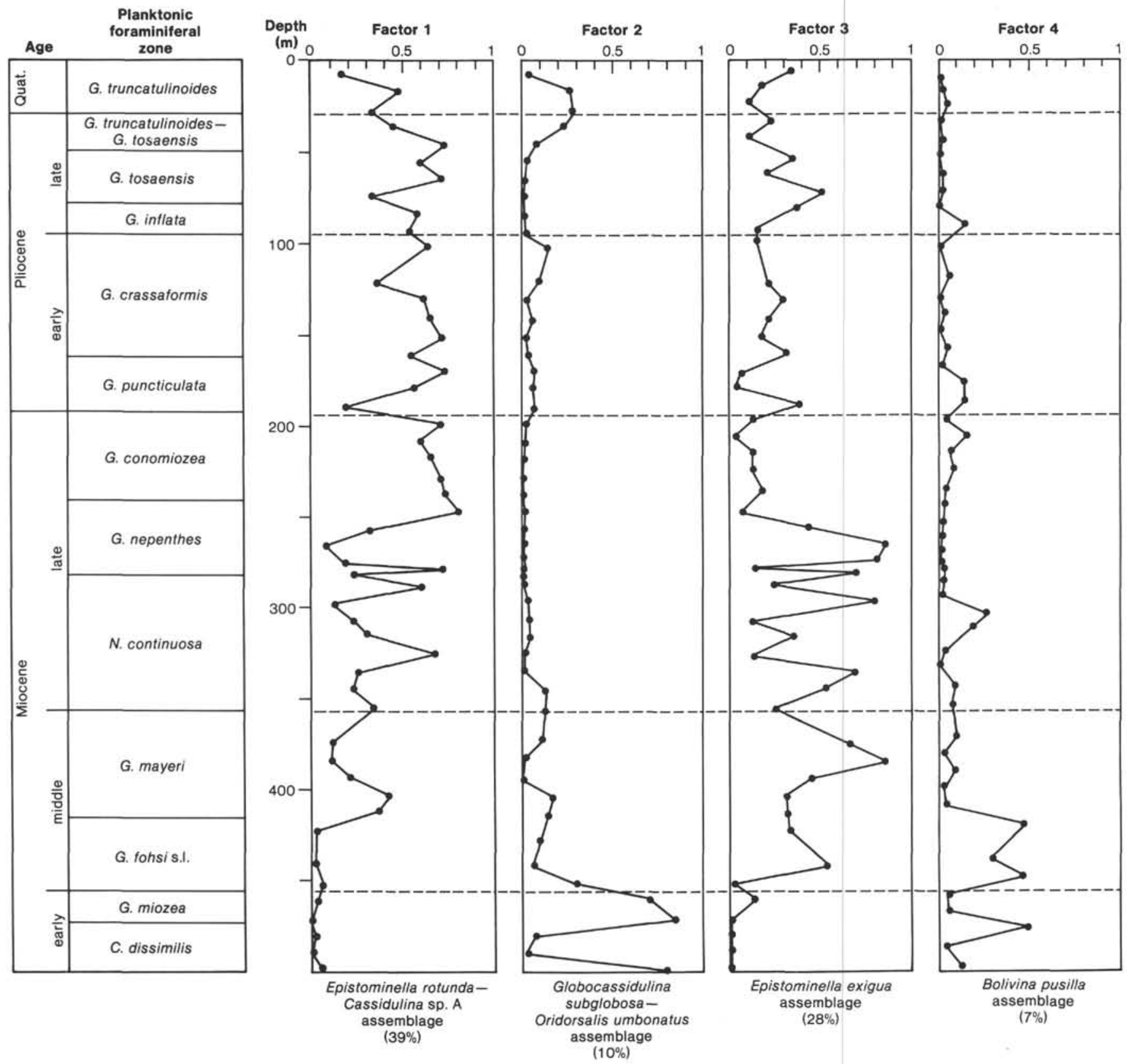

Figure 7. Factor analysis scores ( 5 factors analyzed) for Site 590 , showing changes in the relative importance of four benthic foraminiferal assemblages (factors) in $>63 \mu \mathrm{m}$ fraction. Factor 5 was relatively unimportant quantitatively. Percentages indicate the amount of faunal variance within the data set contained within each of the factors. 


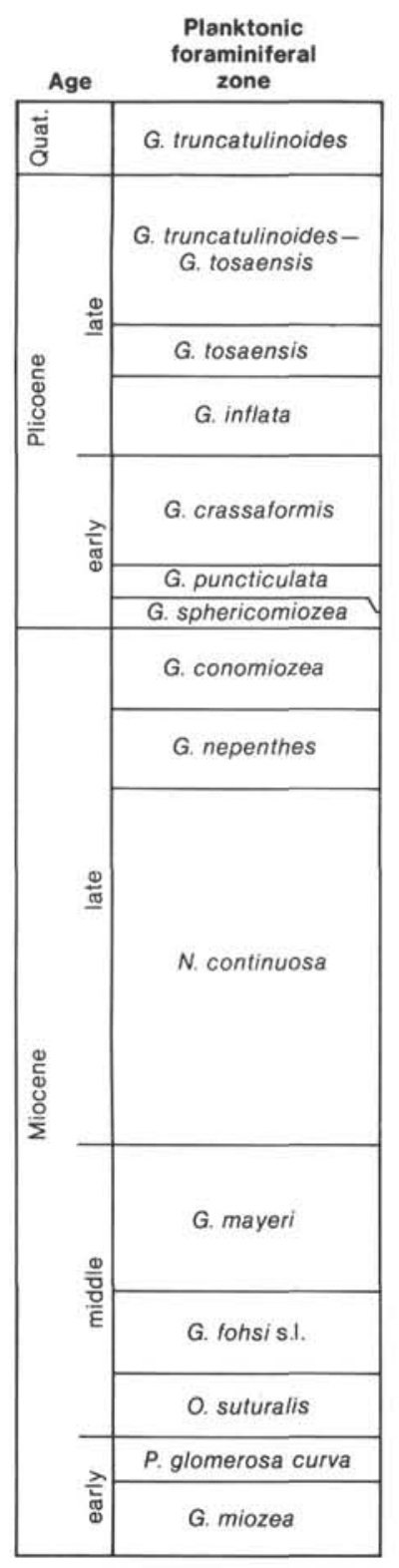
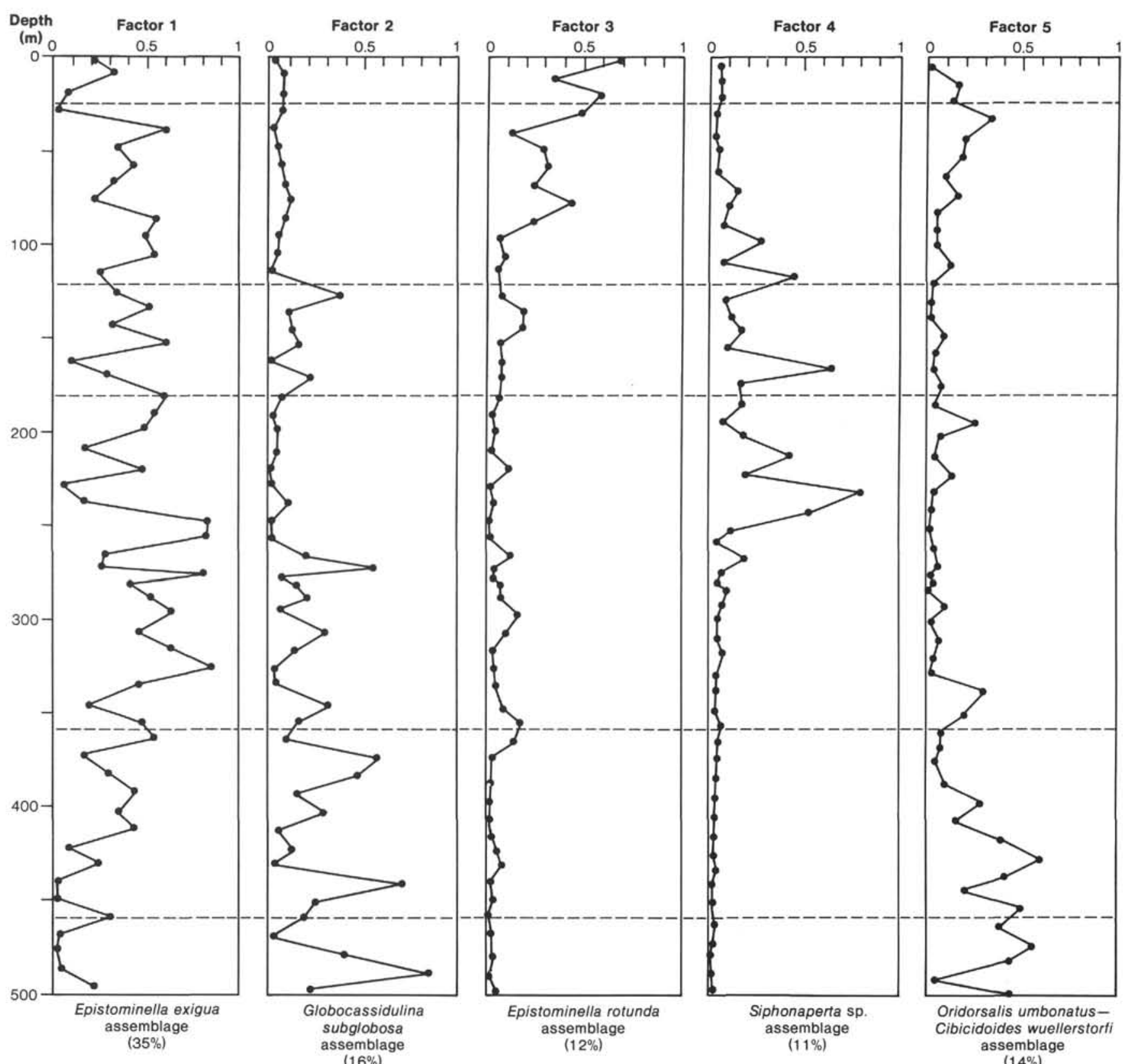

Figure 8. Factor analysis scores ( 5 factors analyzed) for Site 591 , showing changes in the relative importance of five benthic foraminiferal assemblages (factors) in $>63 \mu \mathrm{m}$ fraction. Percentages indicate the amount of faunal variance within the data set contained within each of the factors. 


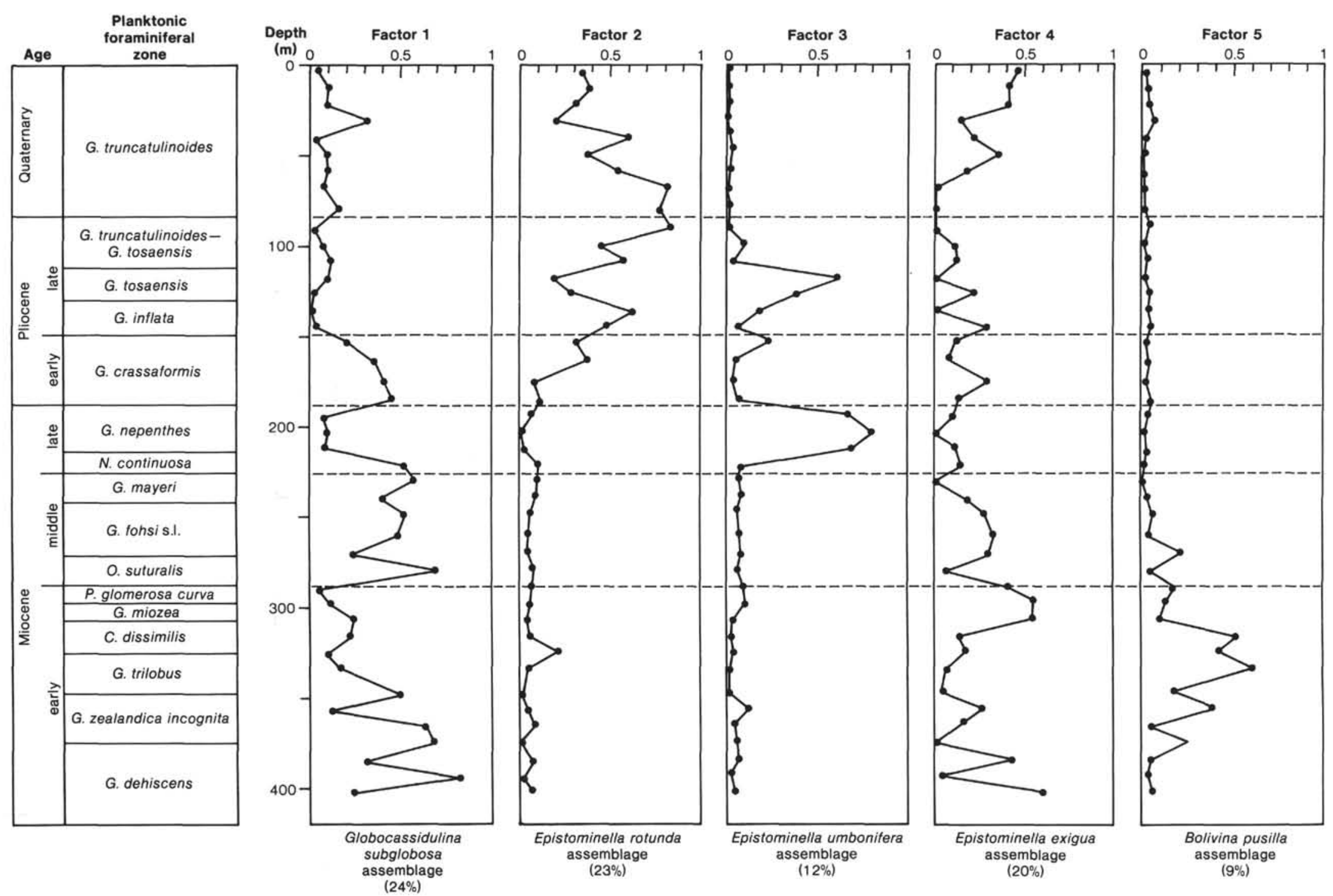

Figure 9. Factor analysis scores ( 5 factors analyzed) for Site 206, showing changes in the relative importance of five benthic foraminiferal assemblages (factors) in $>63 \mu \mathrm{m}$ fraction. Percentages indicate the amount of faunal variance within the data set contained within each of the factors. 


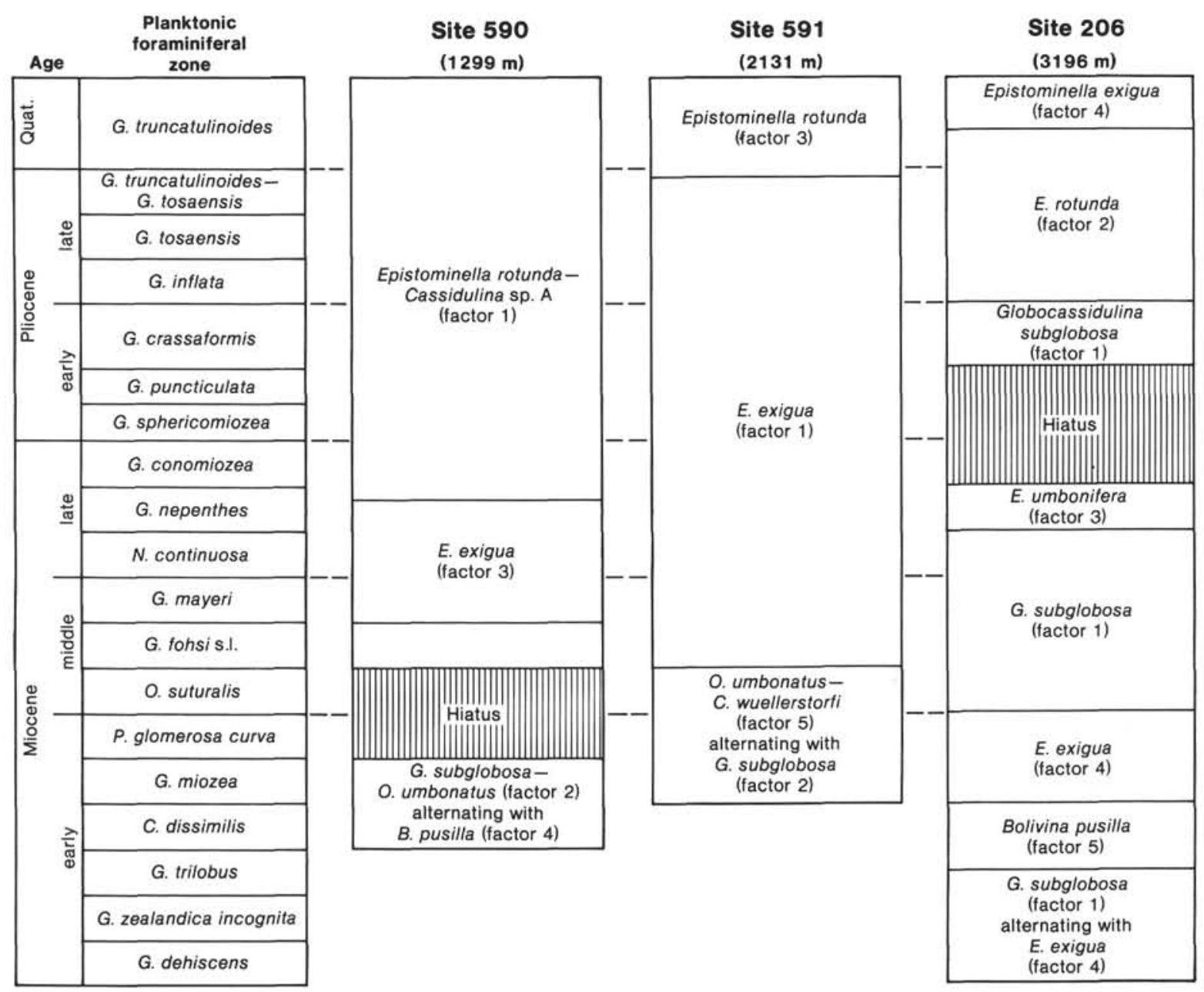

Figure 10. Dominant benthic foraminiferal assemblages at Sites 590, 591, and 206 as revealed by factor analysis (water depth in parentheses). 

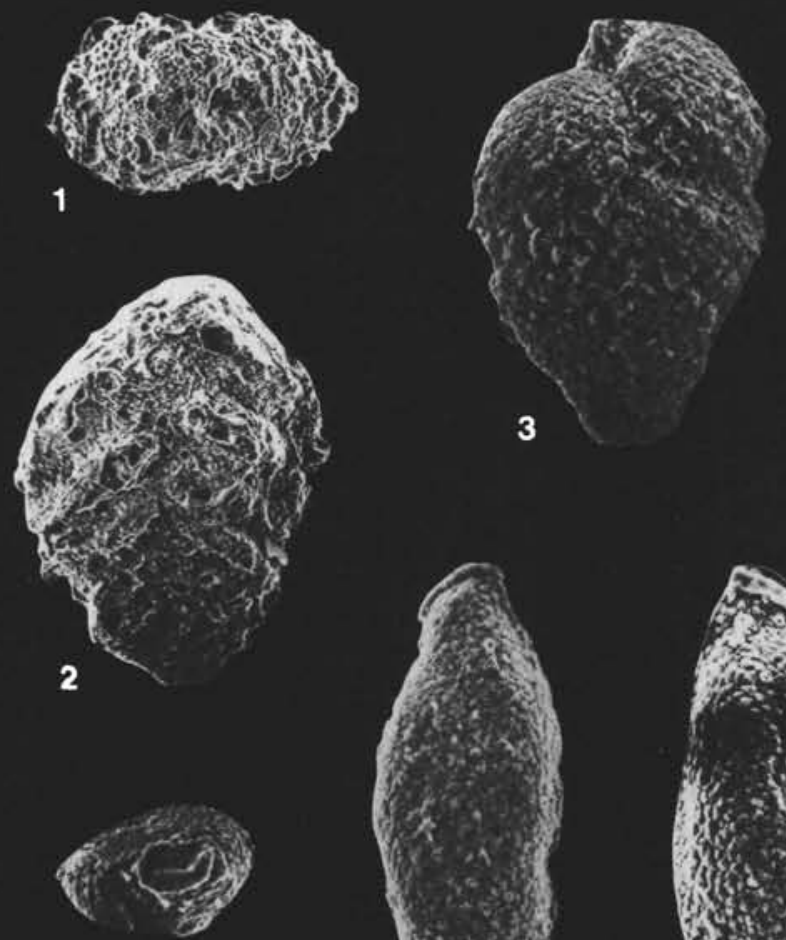

6
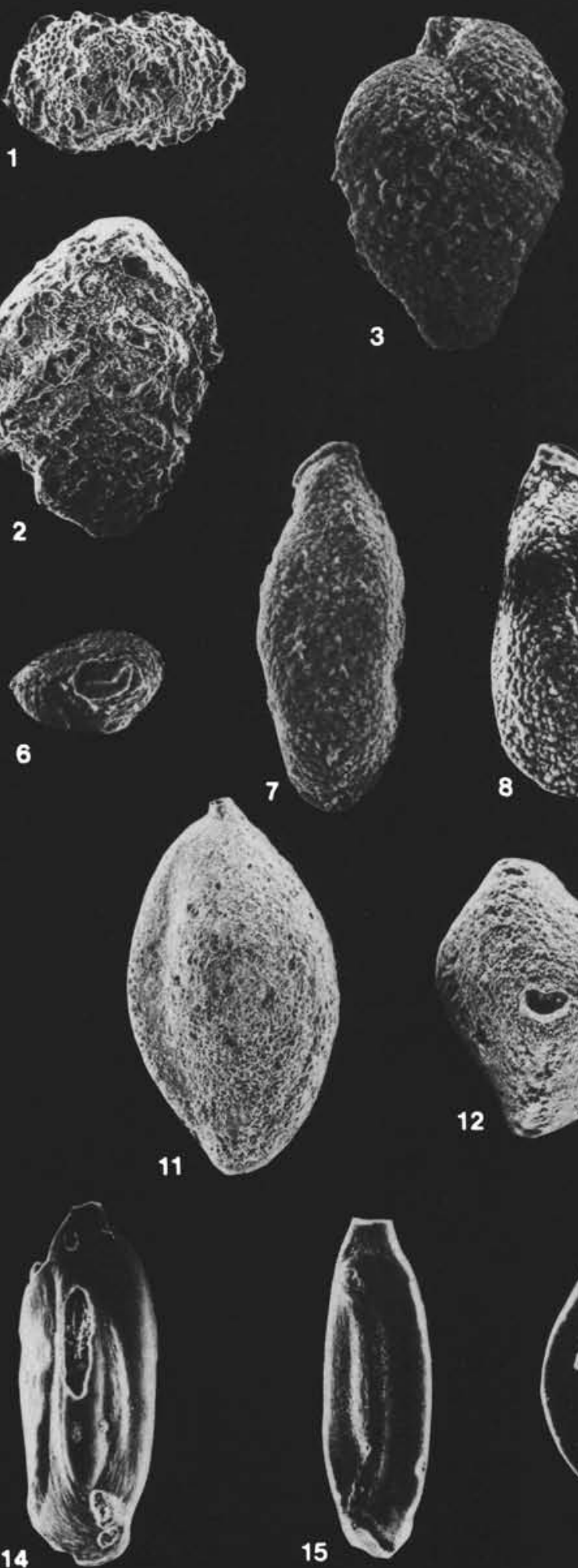
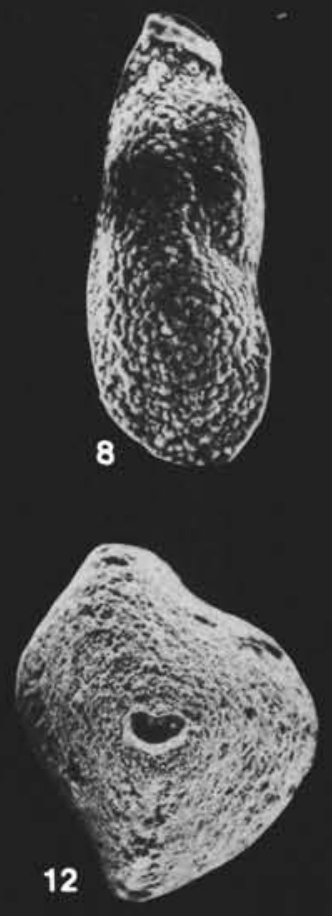

4
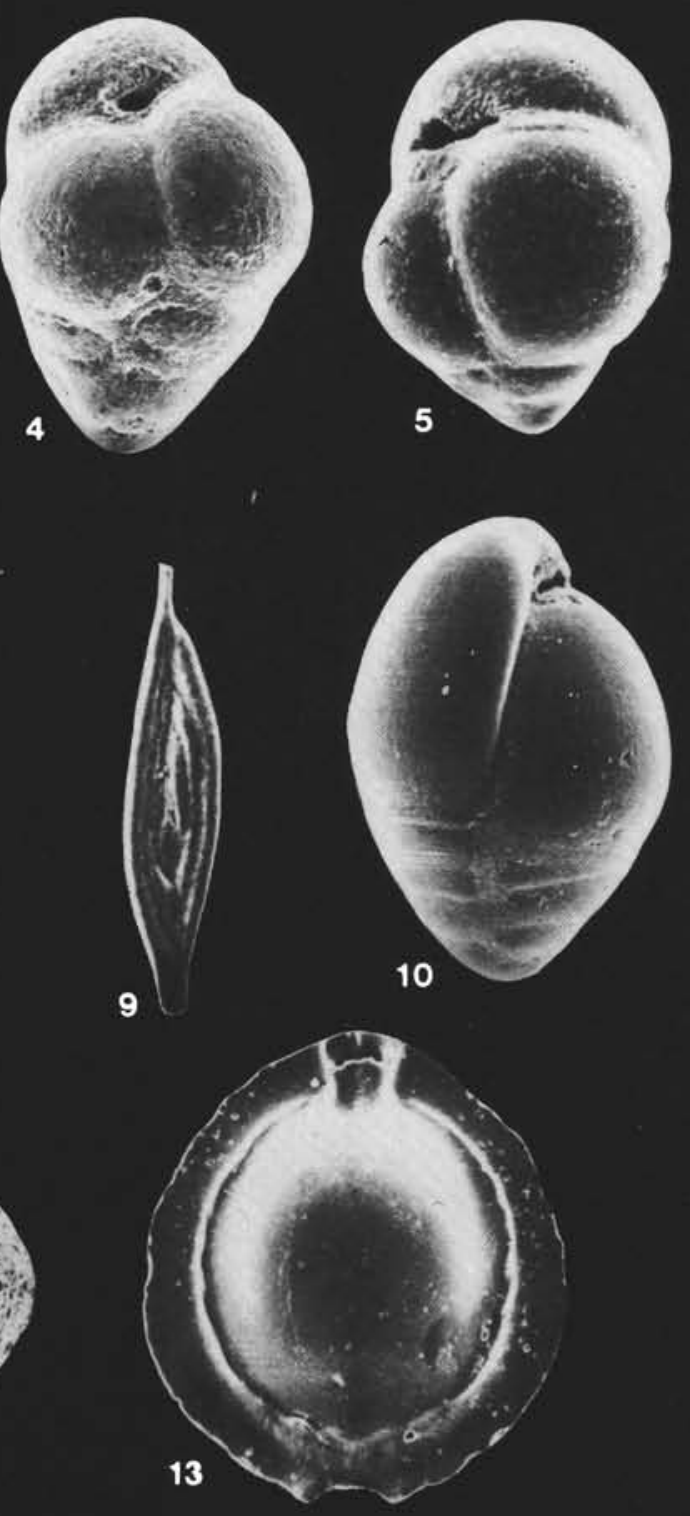
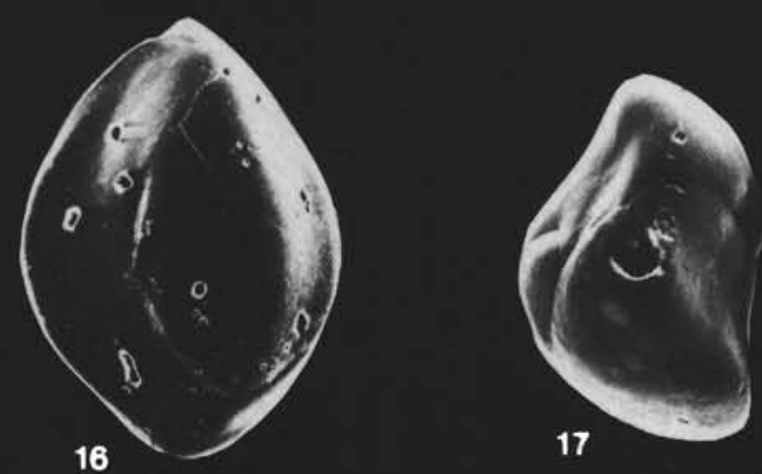

Plate 1. 1, 2. Textularia lythostrota (Schwager), $\times 75$, Sample $591-12$, CC. 3. Siphotextularia rolshauseni Phleger and Parker, $\times 220$, Sample 591-19,CC. 4. Eggerella sp. B, $\times 80$, Sample 591-5,CC. 5. Eggerella sp. A, $\times 133$, Sample 591-5,CC. 6-8. Siphonaperta sp., (6) $\times 513$, Sample 591B-16,CC; (7) $\times 367$, Sample 591-18,CC; $(8) \times 343$, Sample 591-25,CC. 9. Ophthalmidium pusillum (Earland), $\times 96$, Sample 591-4,CC. 10. Dorothia brevis Cushman and Stainforth, $\times 49$, Sample 591-5,CC. 11, 12. Sigmoilopsis schlumbergeri (Silvestri), (11) $\times 70$; (12) $\times 125$, Sample 591-3,CC. 13. Pyrgo murrhina (Schwager), $\times 160$, Sample $591-5$, CC. 14, 15. Quinqueloculina venusta Karrer, (14) $\times 120$, Sample 591-21,CC; (15) $\times 133$, Sample 591-25,CC. 16, 17. Sigmoilina edwardsi $($ Schlumberger), $(16) \times 133 ;(17) \times 213$, Sample $591-1$, CC. 

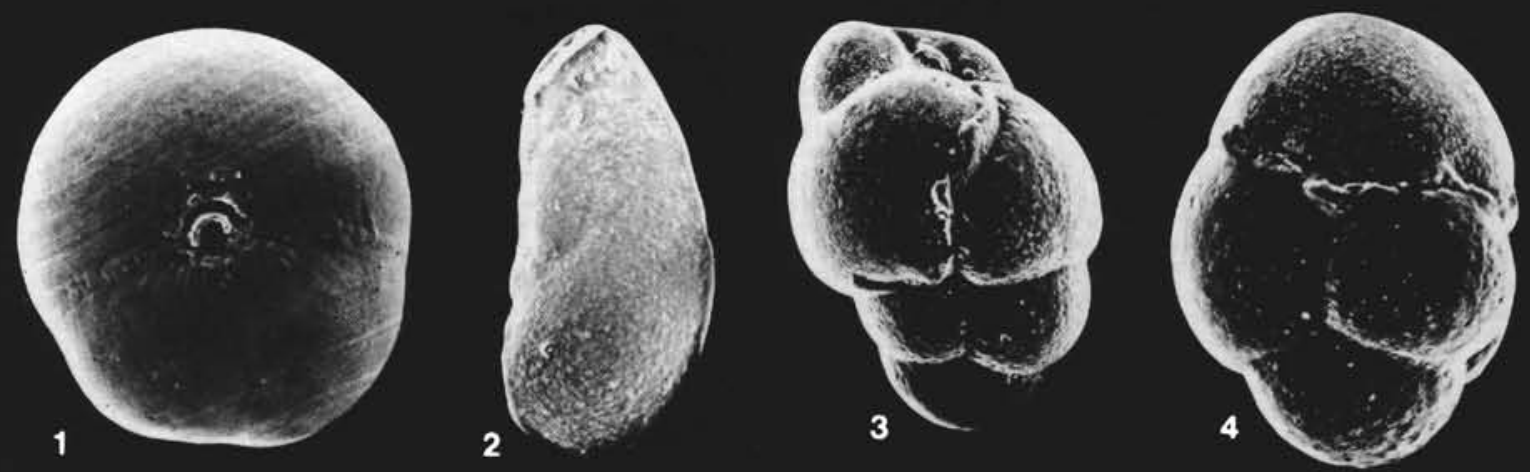

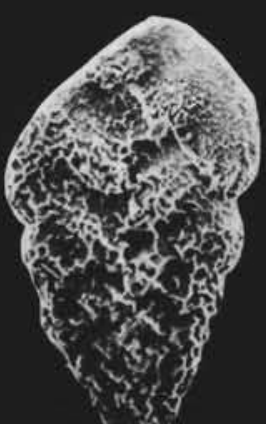

5
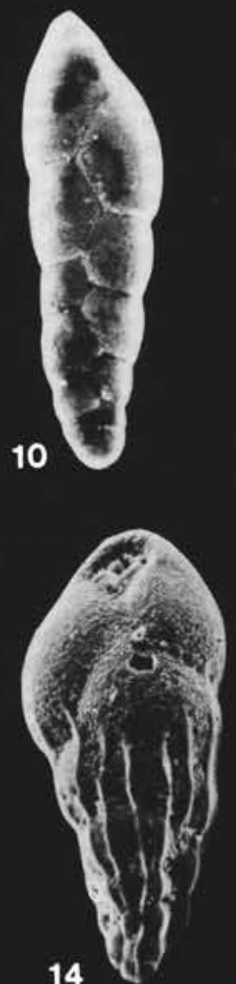
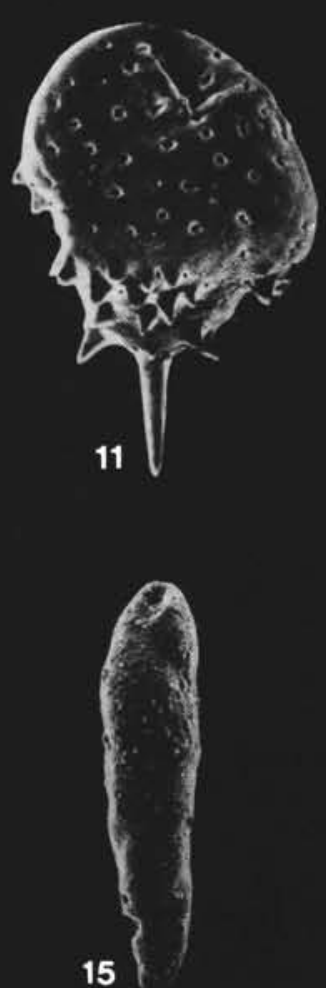

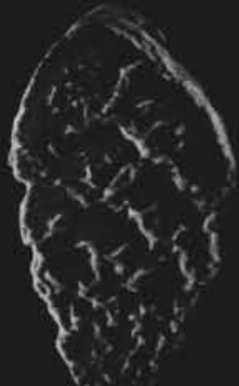

6
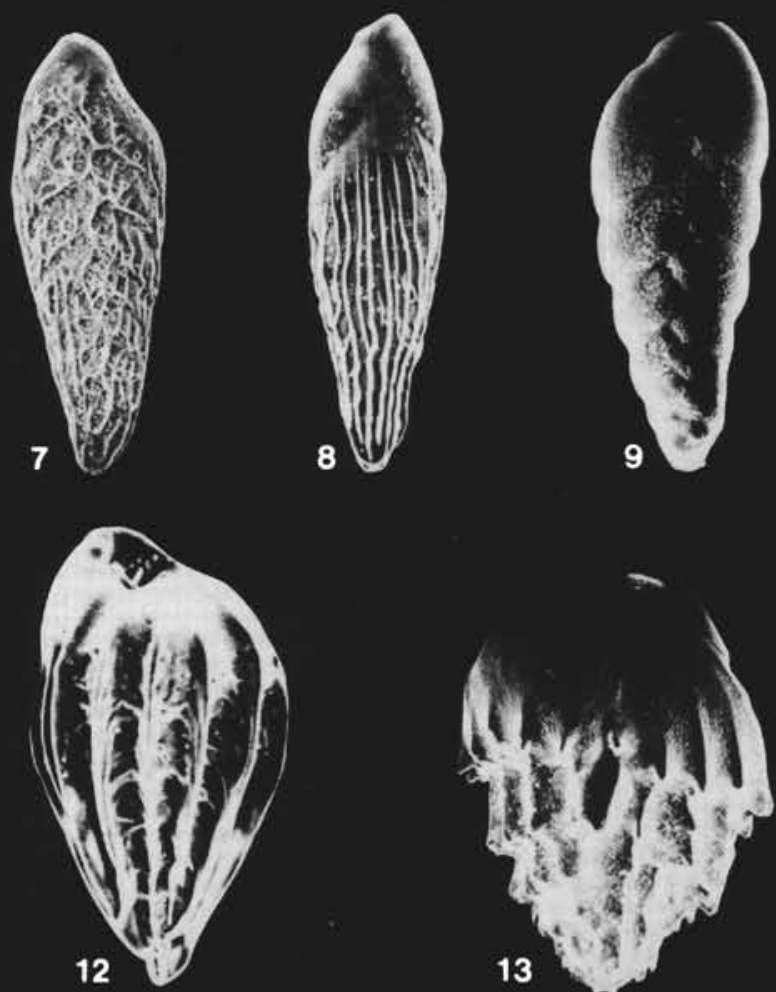

-

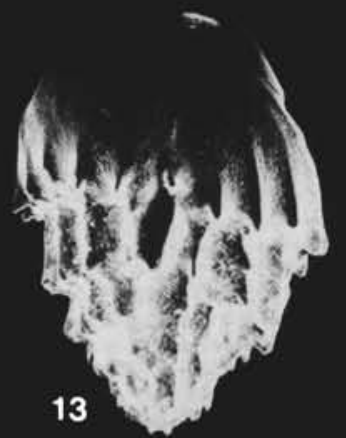

13
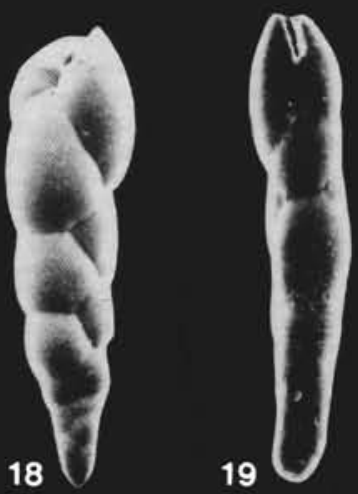

Plate 2. 1. Sphaeroidina bulloides d'Orbigny, $\times 95$, Sample 591-6,CC. 2. Seabrookia earlandi Wright, $\times 285$, Sample $591-21, C C$. 3, 4. Buliminella sp., (3) $\times 199$; (4) $\times 238$, Sample 206-13,CC. 5. Bolivina decussata Brady, $\times 176$, Sample $590 \mathrm{~A}-1$, CC. 6. Bolivina minuta Natland, $\times 249$, Sample 590A-1,CC. 7, 8. Bolivina pusilla Schwager, (7) $\times 117$, Sample 590B-36,CC; $(8) \times 113$, Sample 591-5,CC. 9, 10. Bolivina seminuda Cushman, (9) $\times 108$, Sample 591-3,CC; (10) $\times 120$, Sample 591-5,CC. 11. Bulimina aculeata d'Orbigny, $\times 279$, Sample $590 \mathrm{~A}-1$, CC. 12. Bulimina rostrata Brady, $\times 176$, Sample $591-5$, CC. 13. Bulimina striata d'Orbigny, $\times 83$, Sample $591-3$,CC. 14. Bulimina translucens Parker, $\times 274$, Sample 206-38,CC. 15, 16. Virgulopsis sp., (15) $\times 132$, Sample 590B-52,CC; (16) $\times 220$, Sample 590A-11,CC. 17 . Stainforthia complanata (Egger), $\times 135$, Sample 591-5,CC. 18, 19. Cassidella bradyi (Cushman), (18) $\times 50$; (19) $\times 44$, Sample 591-5,CC. 


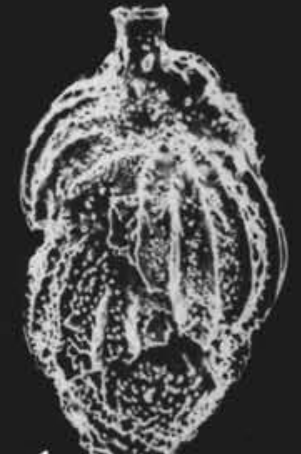

1
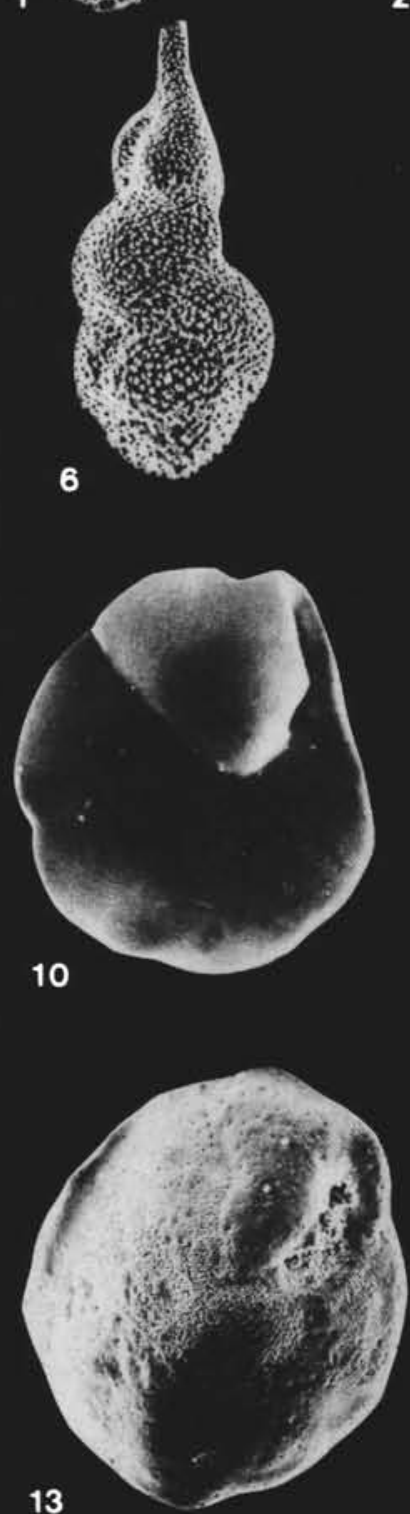
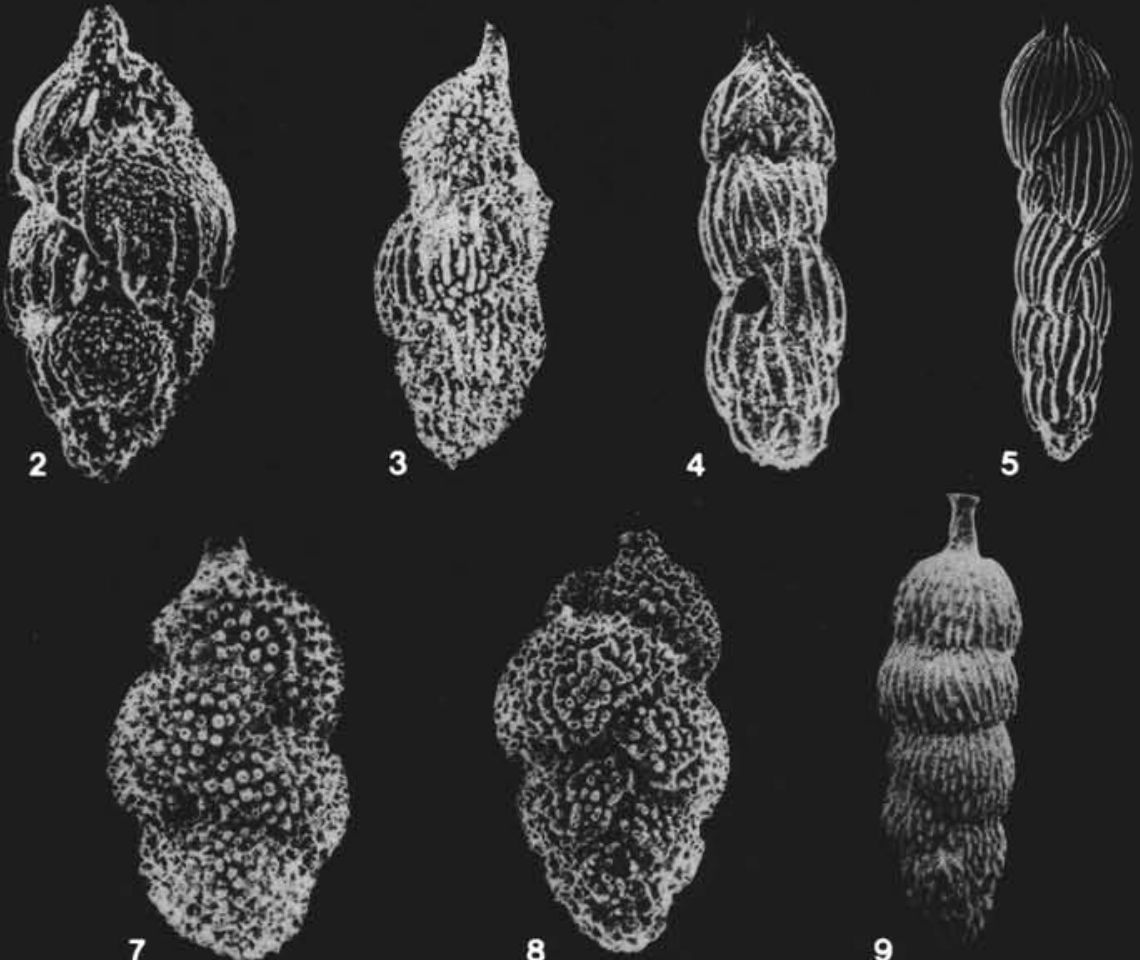

5

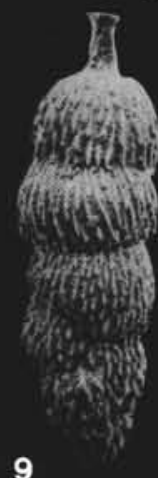

8
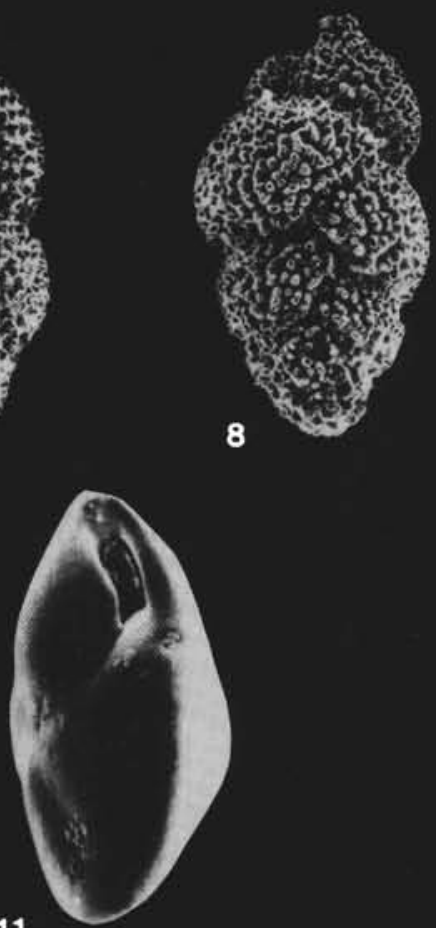

11

12
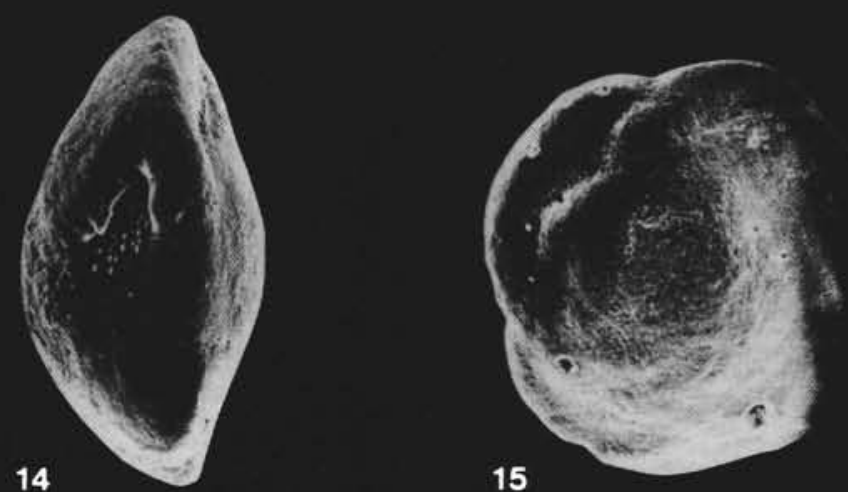

15

Plate 3. 1-3. Uvigerina peregrina Cushman, (1) $\times 107$; (2) $\times 93$; (3) $\times 67$, Sample 591-5,CC. 4, 5. Uvigerina sp. A, (4) $\times 133$, Sample 591-27,CC; (5) $\times 69$, Sample 591-9,CC. 6. Uvigerina proboscidea Schwager, $\times 103$, Sample 591-5,CC. 7, 8. Uvigerina hispida Schwager, (7) $\times 63 ;(8) \times 64$, Sample 591-24,CC. 9. Rectuvigerina sp, $\times 73$, Sample 590A-27,CC. 10-12. Epistominella exigua (Brady), $(10) \times 227 ;(11) \times 213 ;(12) \times 175$, Sample 591-1,CC. 13-15. Epistominella rugosa (Phleger and Parker), (13) $\times 128$; (14) $\times 266$; (15) $\times 208$, Sample 206-13, CC. 

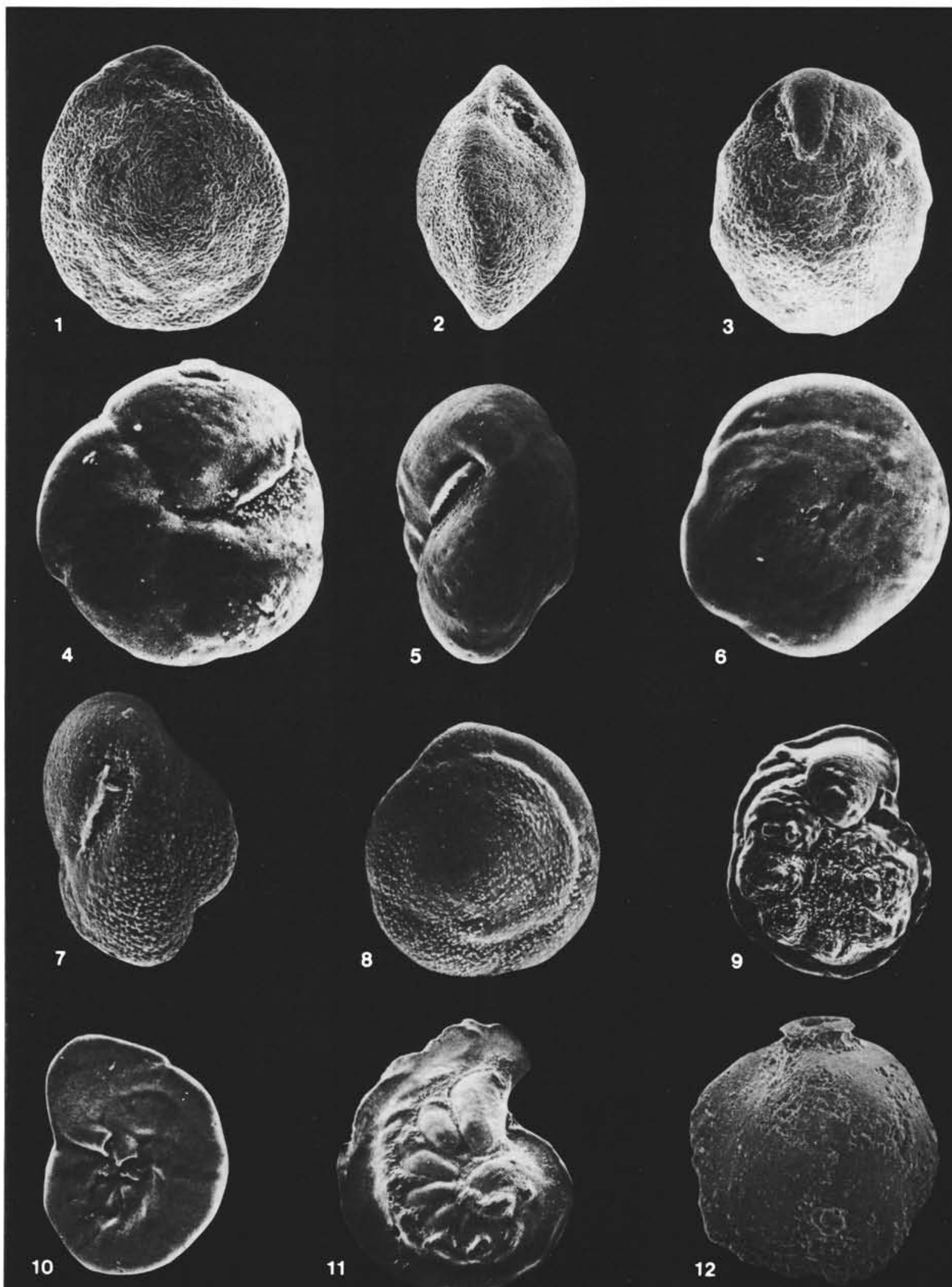

\section{8}
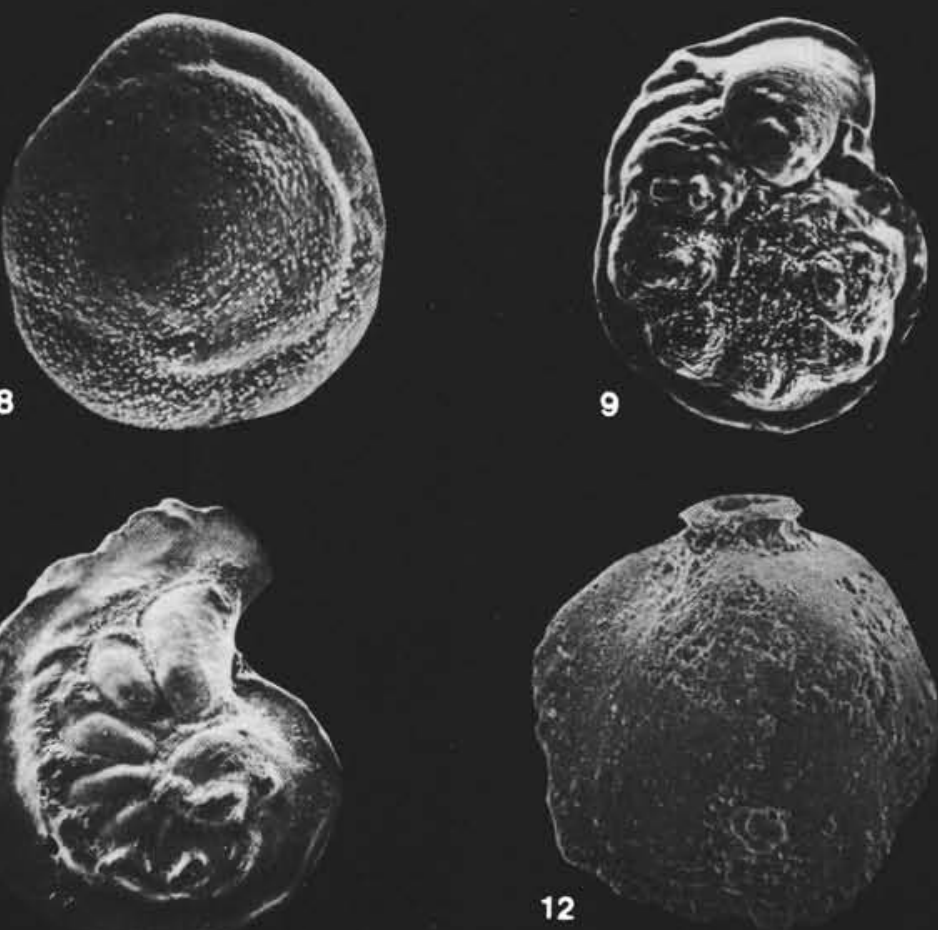

Plate 4. 1-3. Epistominella umbonifera (Cushman), (1) $\times 100 ;(2) \times 117 ;(3) \times 91$, Sample 591B-15,CC. 4-8. Epistominella rotunda (Husezima and Maruhashi) (=Eponides rotundus Husezima and Maruhashi, 1944), (4) $\times 387 ;(5) \times 479 ;(6) \times 383$, Sample 591-1,CC, (7) $\times 383 ;(8) \times 367$, Sample 590A-14,CC. 9,10. Laticarinina alto-camerata (Heron-Allen and Earland), $(9) \times 100 ;(10) \times 126$, Sample 591-1,CC. 11. Laticarinina pauperata (Parker and Jones), $\times 79$, Sample 206-11,CC. 12. Siphonina australis Cushman, $\times 112$, Sample 591B-24,CC. 

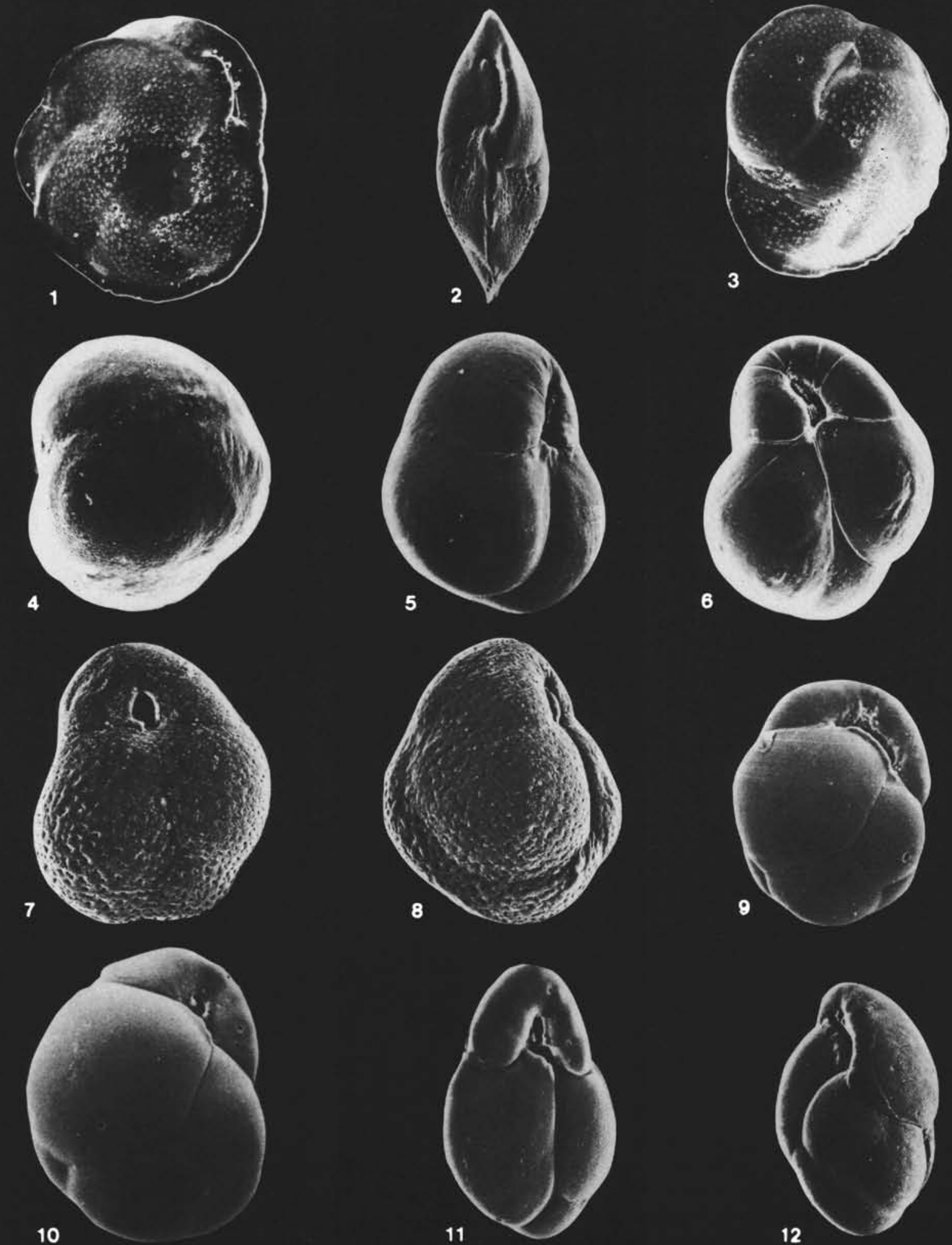

Plate 5. 1-3. Cassidulina carinata Silvestri, (1) $\times 144$, Sample 591-5,CC; (2) $\times 144$, Sample 591-6,CC; (3) $\times 184$, Sample 591-5,CC. 4-8. Globocassidulina subglobosa (Brady), (4) $\times 95 ;(5) \times 119$; (6) $\times 110$, Sample 591-5,CC, (7) $\times 400 ;(8) \times 466$, Sample 591-18,CC. 9, 12. Cassidulina sp. B $\times 208$, Sample 591-5,CC. 10, 11. Cassidulina sp. A, $\times 364$, Sample 591-3,CC. 

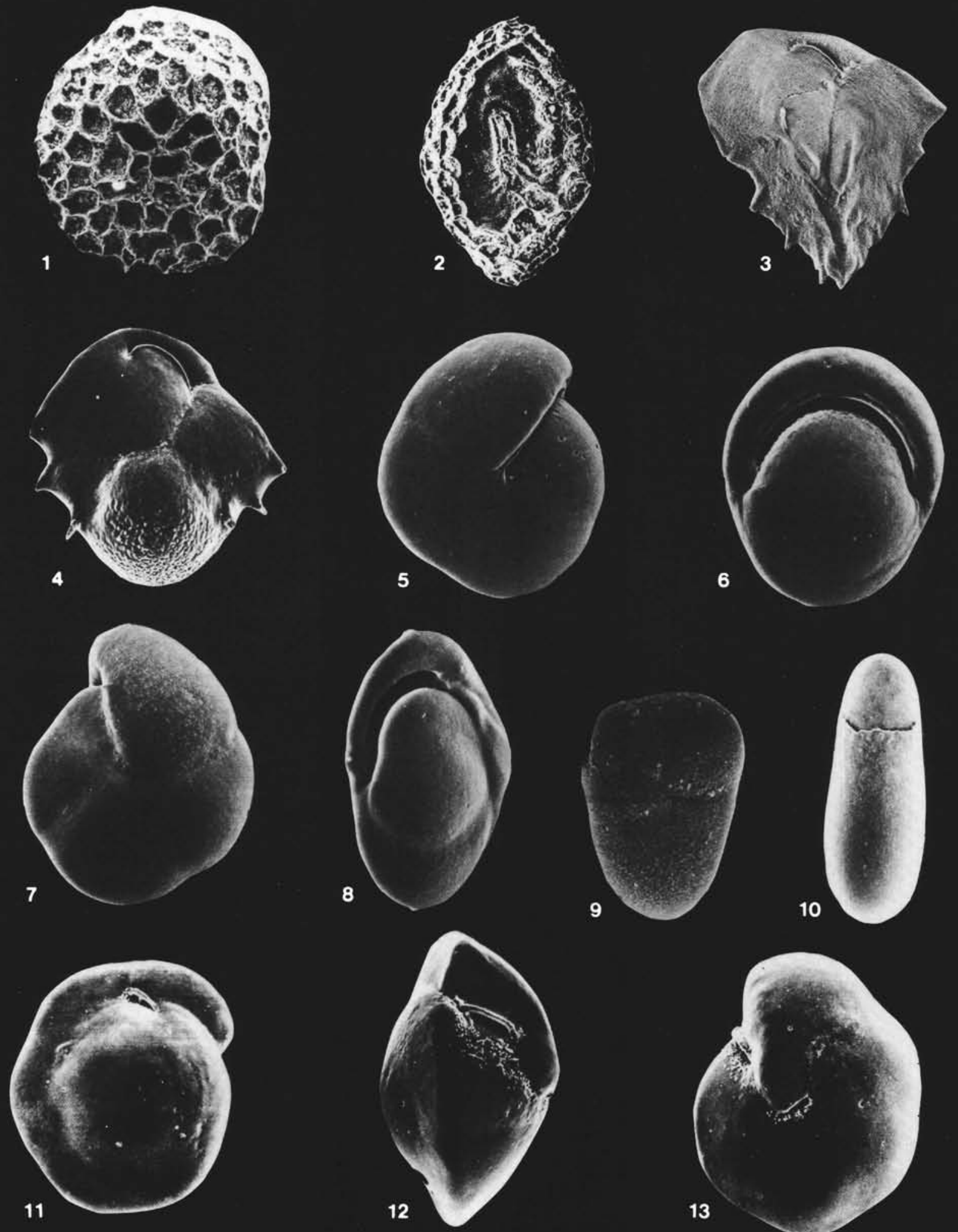

Plate 6. 1, 2. Favocassidulina favus (Brady), $\times 141$, Sample 206-19,CC. 3. Ehrenbergina trigona Goës, $\times 147$, Sample $590 \mathrm{~A}-27, \mathrm{CC} . \quad$ 4. Ehrenbergina sp., $\times 125$, Sample 591-10,CC. 5, 6. Pullenia bulloides (d'Orbigny), $(5) \times 163 ;(6) \times 180$, Sample $591-5$, CC. 7, 8. Pullenia osloensis Feyling-Hanssen, (7) $\times 249$; (8) $\times 323$, Sample 590A-1,CC. 9. Allomorphina pacifica Cushman and Todd, $\times 147$, Sample $590 \mathrm{~B}-43$, CC. 10 . Chilostomella oolina Schwager, $\times 104$, Sample 206-5,CC. 11-13. Oridorsalis umbonatus (Reuss), $(11) \times 100 ;(12) \times 115 ;(13) \times 127$, Sample 591-5,CC. 

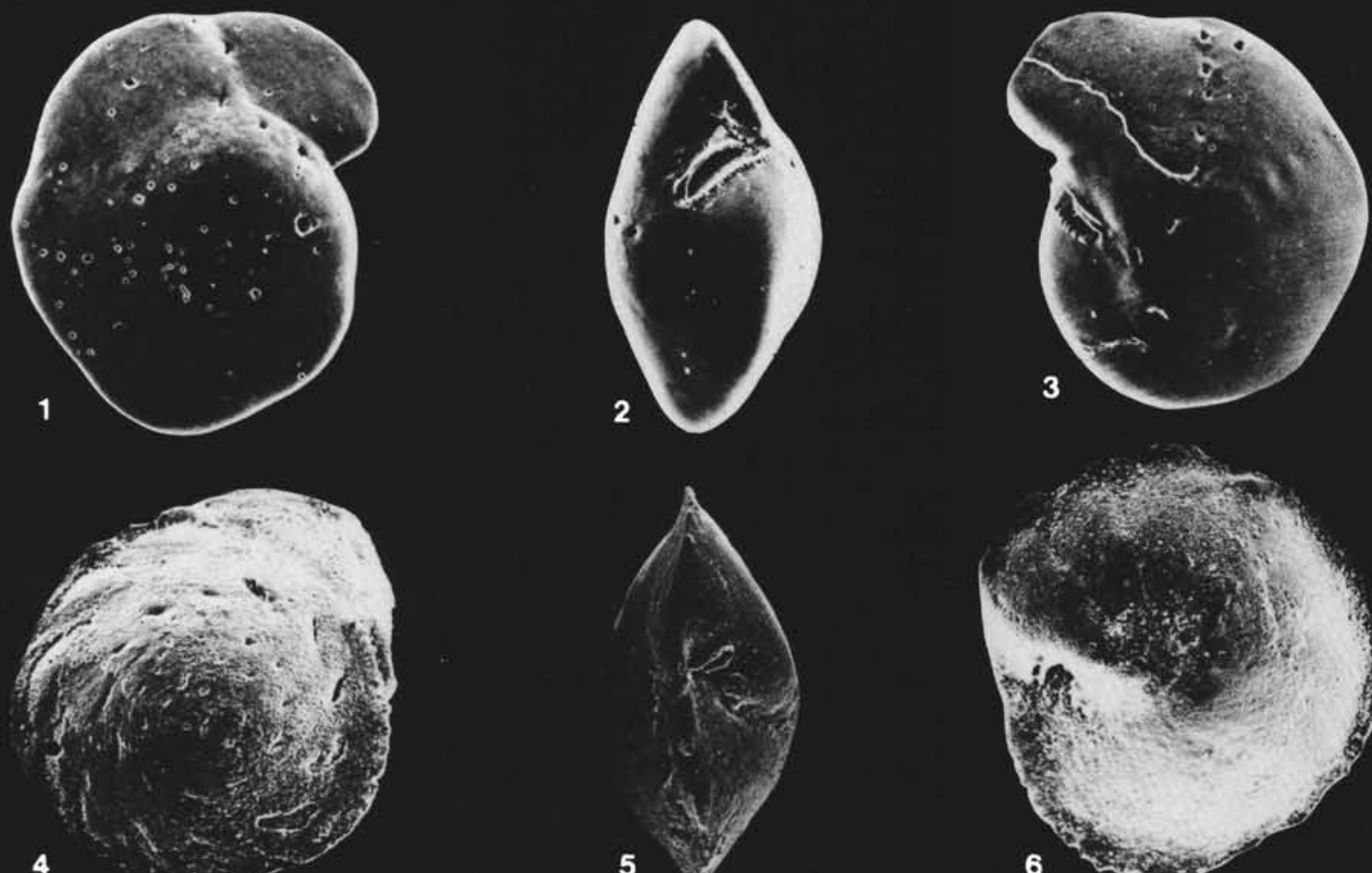

4
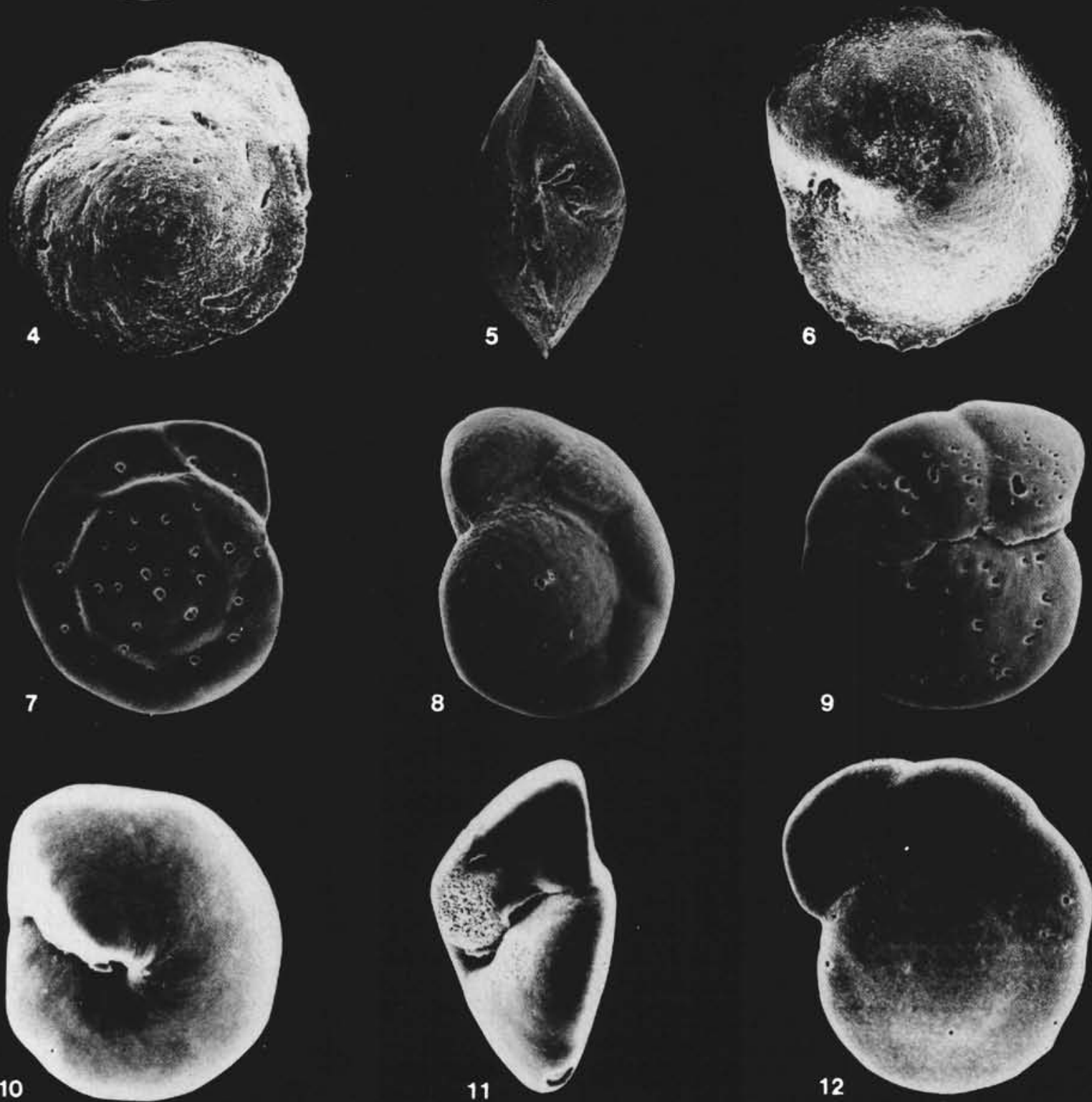

Plate 7. 1-3. Oridorsalis sp. $(1,2) \times 93 ;(3) \times 96$, Sample 591-5,CC. 4-6. Osangularia culter (Parker and Jones), (4) $\times 105 ;(5) \times 125 ;(6) \times 123$, Sample 591-14,CC. 7. Gyroidina acuta Boomgaart, $\times 73$, Sample 591-7,CC. 8. Gyroidina neosoldanii Brotzen, $\times 61$, Sample 591-7,CC. 9. Anomalinoides sp. A, $\times 213$, Sample 591-6,CC. 10-12. Gyroidina sp. A, (10) $\times 207$; (11) $\times 230$; (12) $\times 240$, Sample $591-5$,CC. 


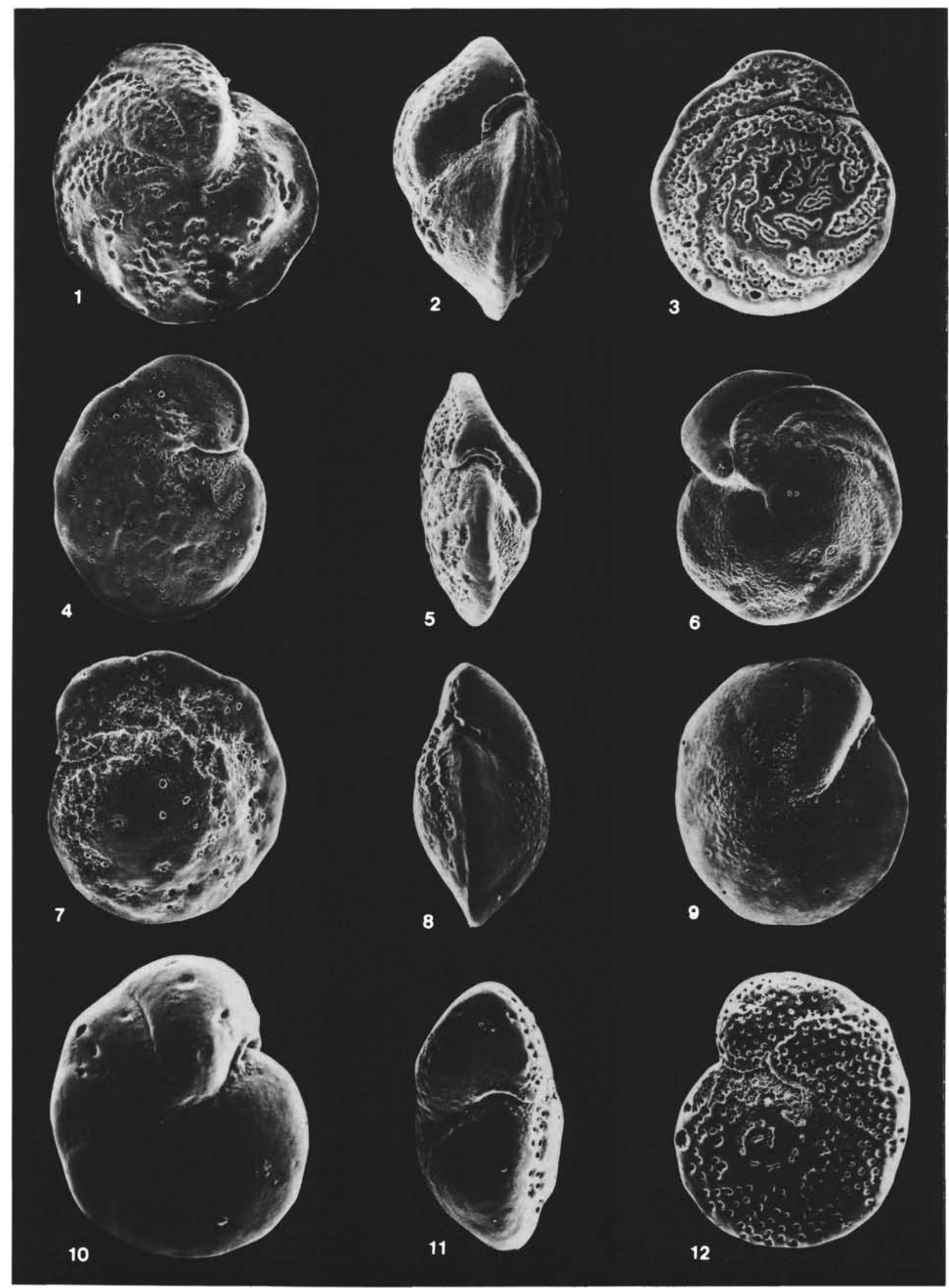

Plate 8. 1-3. Cibicidoides kullenbergi (Parker), (1) $\times 80 ;(2) \times 74 ;(3) \times 69$, Sample 591-14,CC. 4-6. Cibicidoides wuellerstorfi (Schwager), (4, 5) $\times 74$; (6) $\times 68$, Sample 591-8,CC. 7-9. Cibicidoides mundulus (Brady, Parker, and Jones), $\times 107$, (7, 9) Sample 591-11,CC; (8) Sample 591-10,CC. 10-12. Cibicidoides sp. A, (10) $\times 110$; (11) $\times 122 ;(12) \times 100$, Sample 591-6,CC. 

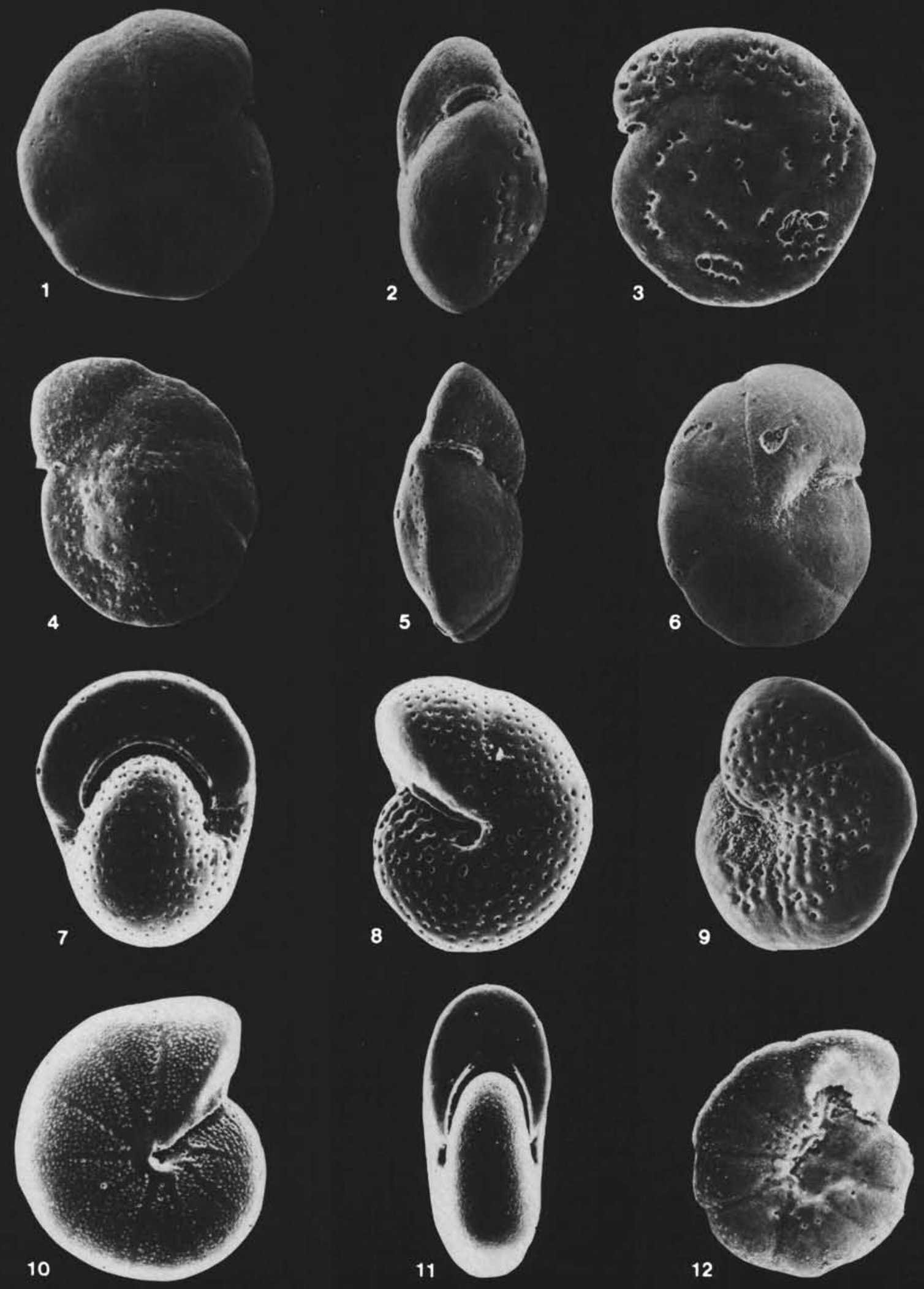

Plate 9. 1-3. Cibicidoides bradyi (Trauth), (1) $\times 81$; (2) $\times 152$; (3) $\times 129$, Sample 591-6,CC. 4-6. Cibicidoides $\mathrm{cf}$. opacus $($ Carter), (4) $\times 137$; (5) $\times 190$; (6) $\times 161$, Sample 591B-15,CC. 7, 8. Melonis pompilioides (Fichtel and Moll), $(7) \times 161 ;(8) \times 173$, Sample 591-5,CC. 9. Anomalinoides globulosus (Chapman and Parr), $\times 148$, Sample 591-8,CC. 10, 11. Melonis barleeanum (Williamson), $(10) \times 132 ;(11) \times 147$, Sample 591-5,CC. 12. Anomalinoides sp. B, $\times 375$, Sample 590-1,CC. 